University of Louisville ThinkIR: The University of Louisville's Institutional Repository

Electronic Theses and Dissertations

$5-2019$

\title{
Urban-ready, willing, and able? Collaborative induction experiences for new urban elementary educators.
}

Sandra Lynn Hogue

University of Louisville

Follow this and additional works at: https://ir.library.louisville.edu/etd

Part of the Educational Leadership Commons, Elementary Education Commons, Elementary Education and Teaching Commons, and the Other Teacher Education and Professional Development Commons

\section{Recommended Citation}

Hogue, Sandra Lynn, "Urban-ready, willing, and able? Collaborative induction experiences for new urban elementary educators." (2019). Electronic Theses and Dissertations. Paper 3160.

https://doi.org/10.18297/etd/3160

This Doctoral Dissertation is brought to you for free and open access by ThinkIR: The University of Louisville's Institutional Repository. It has been accepted for inclusion in Electronic Theses and Dissertations by an authorized administrator of ThinkIR: The University of Louisville's Institutional Repository. This title appears here courtesy of the author, who has retained all other copyrights. For more information, please contact thinkir@louisville.edu. 


\title{
URBAN-READY, WILLING, AND ABLE? COLLABORATIVE INDUCTION EXPERIENCES FOR NEW URBAN ELEMENTARY EDUCATORS
}

\author{
By \\ Sandra Lynn Hogue \\ B.S., University of Louisville, 1995 \\ M.Ed., University of Louisville, 2000 \\ Rank I, University of Louisville, 2008
}

\begin{abstract}
A Dissertation
Submitted to the Faculty of the

College of Education and Human Development of the University of Louisville In Partial Fulfillment of the Requirements

For the Degree of
\end{abstract}

Doctor of Education

in Educational Leadership and Organizational Development

Department of Education Leadership, Evaluation, and Organizational Development University of Louisville

Louisville, Kentucky

May 2019 
Copyright 2019 by Sandra Lynn Hogue

All rights reserved 

URBAN-READY, WILLING, AND ABLE?

COLLABORATIVE INDUCTION EXPERIENCES FOR NEW URBAN

\section{ELEMENTARY EDUCATORS}

$$
\text { By }
$$

Sandra Lynn Hogue

B.S., University of Louisville, 1995

M.Ed., University of Louisville, 2000

Rank I, University of Louisville, 2008

\section{A Dissertation Approved on}

February 25, 2019

by the following Dissertation Committee:

Dissertation Director

Mary Brydon-Miller

Blake Haselton

Detra Johnson

Marco A. Muñoz 


\section{DEDICATION}

This dissertation is dedicated to my children, parents, siblings, and loving supporters:

\section{Rhaegan Grace Hogue and Grayson Reign Hogue,}

Aaron Othel Hogue and Lily Pearl Hogue,

Antonio Hogue, Sheba Waters, Adrian Harris, Aaron Hogue, Stephanie Wells, and Candice Hogue,

and all of whom have encouraged and supported my persistence and determination to live a \#BlessedLife. 


\section{ACKNOWLEDGEMENTS}

I give all praises and honor to my Lord and Savior, Jesus Christ who continues to bless my life. I acknowledge that without Him, I am nothing, but with Him, I can do amazing things. I thank Him publicly and privately for all He has been, is, and will continue to be in my life.

I would like to thank each of my dissertation committee members for believing in and investing in me. I would not be here without you; Dr. Brydon-Miller, for your honest, responsive guidance throughout this arduous process, Dr. Johnson, for your tough love that set a tone for excellence, Dr. Muñoz, for your encouraging spirit from start to finish, and for guidance in navigating the district, and Dr. Haselton, for coaching that was timely and thorough. This journey has been enriched by each of you. Thank you for the blessings.

I would like to also thank each of the following people who have, in their own critical ways, uplifted me and my work over the course of this journey and beyond. Your support is appreciated. Many thanks to each of you; The Hogue Family (for everything), Andre Howard, Jean Wolph, Missy Callaway, Shelli Barber, Lori Trout, Jean Anne Clyde, Chuck Woodall, Nyree Clayton-Taylor, Harvetta Broadway-Ray, and my church family. A final special acknowledgment to my father, Mr. Aaron Othel Hogue, who did 
not live to see the completion of this journey but is celebrating me on high. This is truly a \#BlessedLife. 


\begin{abstract}
URBAN-READY, WILLING, AND ABLE?

COLLABORATIVE INDUCTION EXPERIENCES FOR NEW URBAN EDUCATORS

Sandra Lynn Hogue
\end{abstract}

February 25, 2019

This qualitative study explored the lived experiences of six first year urban educators who participated in a series of multi-stakeholder induction experiences during the first semester of their first year teaching in high-need urban elementary schools. This study underscores how participation in needs-based induction experiences nurture selfefficacy and encourage teacher retention through the challenging first semester of teaching.

Data for this study was collected over a four-week period of new teacher participation in a system of support that included focus group conversations, professional development sessions, documentation of experiences using PhotoVoice, and participant reflective journal entries. The research methodology that structures the study is Abawi's (2015) refractive phenomenology, a method that explores the data through multiple lenses to reach the essence of the participants' lived experiences. This study elucidated the new teachers' experiences prior to the study's induction supports and documented how their perceptions changed over time. The study revealed that new teachers who felt they lacked urban competence benefited from the support of multiple stakeholders 
including university, school district, and the new teachers themselves. The study focused on the new teachers themselves as critical stakeholders in their development of success skills, such as urban competence. The study concluded that new teachers in high-need urban schools benefit from interactions with peers and other stakeholders as they engage in professional learning designed to increase their professional and urban competencies. 
TABLE OF CONTENTS



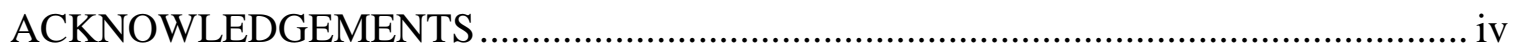

ABSTRACT ...................................................................................................... vi

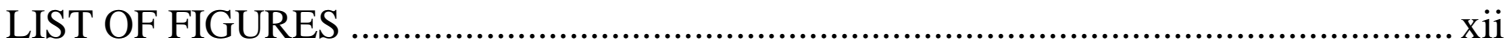



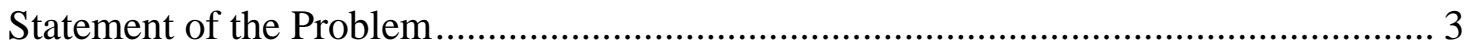

Teacher Shortage ……………………………………................................. 4



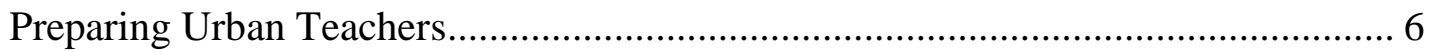

Purpose of the Study ……..................................................................................... 9

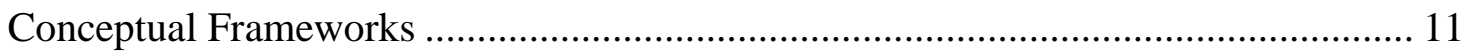

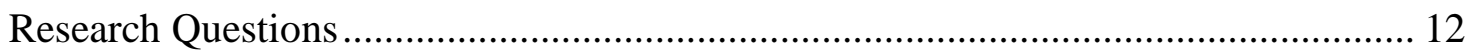



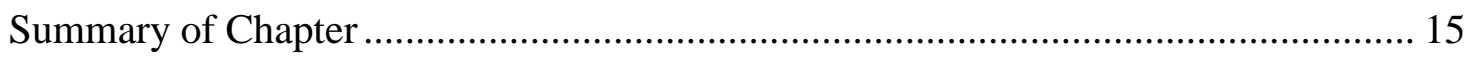

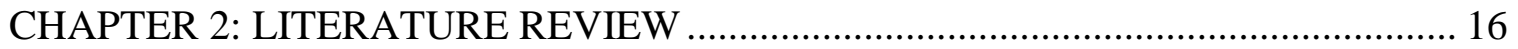

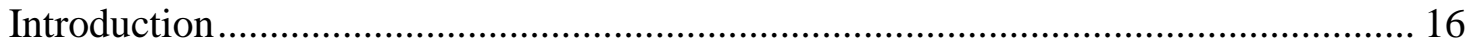

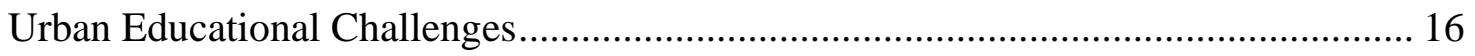

Adverse Childhood Experiences ...................................................................... 18

Poverty and Diversity of Students ....................................................................... 20

Factors Associated with Urban Teacher Attrition ..................................................... 25



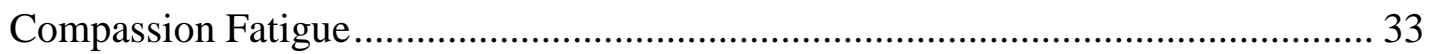

Conceptual Framework: Community Teacher.......................................................... 34

Conceptual Framework: Authentic Teaching …………………………………....... 35

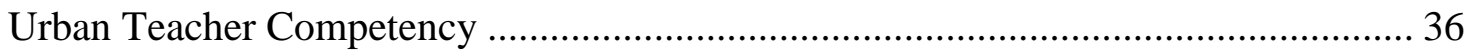





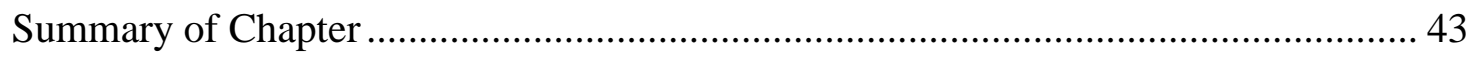






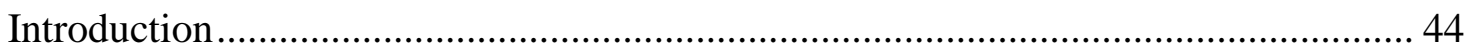

Research Approach and Context........................................................................ 44

Demographics and Context of the Focus School District................................................ 46

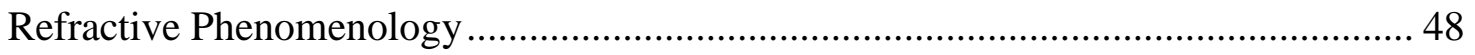

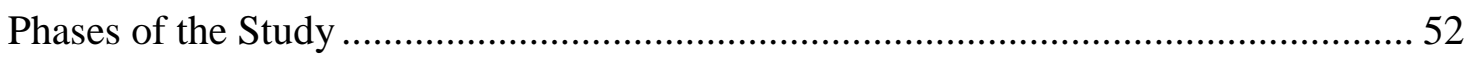

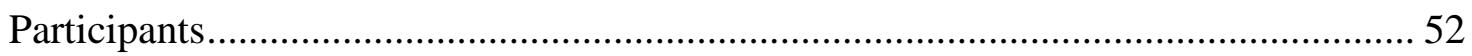

The Multi-stakeholder Induction Experiences ......................................................... 56

Session One: Culture, Poverty, and Community ………………………………..... 57

Session Two: Cultural Competence through Literature............................................ 58

Session Three: Community-Based Resources ....................................................... 58

Session Four: Poverty-Informed Practices............................................................ 59

Session Five: Closing Culture, Poverty, and Community ………………………..... 59

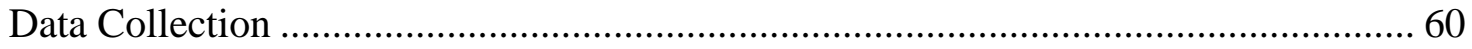

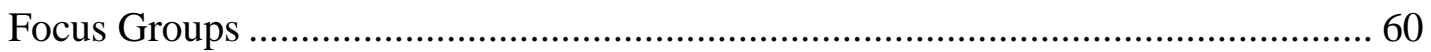

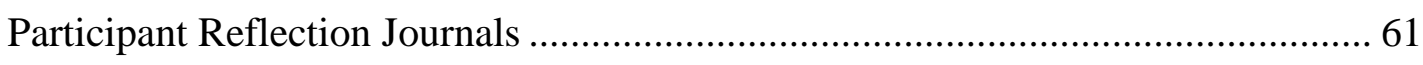







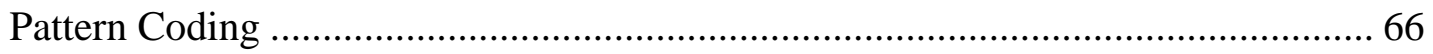



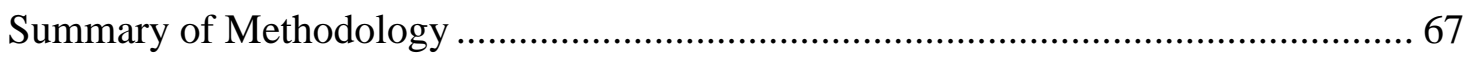







Research Context and Positionality: Researching Self ............................................. 70

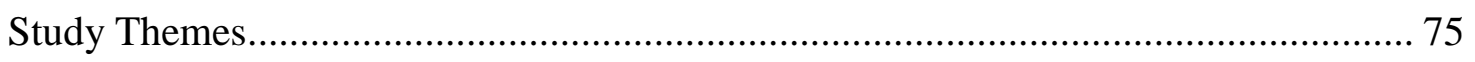

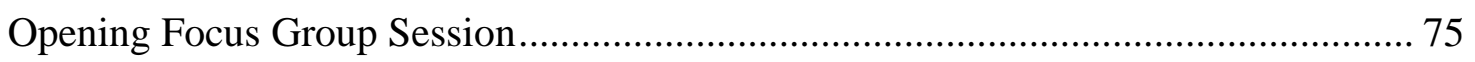

Opening Focus Group: Compassion …………………………………………...... 77

Opening Focus Group: Frustration .................................................................. 78

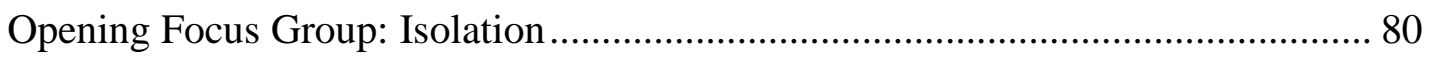

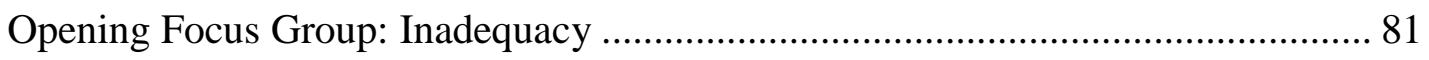

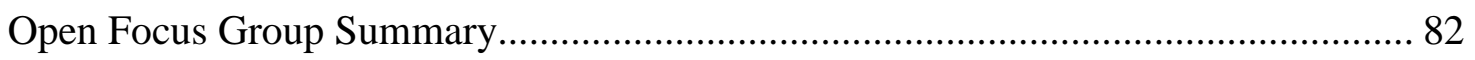




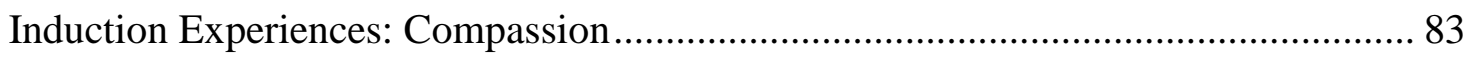

Induction Experiences: Compassion in Weekly Reflective Journal Entry ............... 83

Induction Experiences: PhotoVoice Experience Compassion ................................... 85

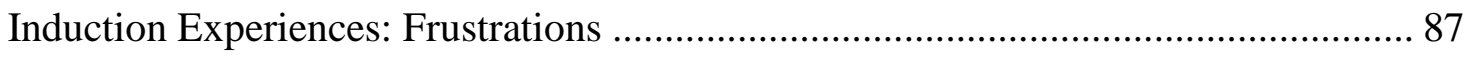

Induction Experiences: Weekly Reflective Journal Entry Frustrations ..................... 87

Induction Experiences: PhotoVoice Experience Frustrations..................................... 92

Summary of Frustration during Induction Experiences............................................. 94

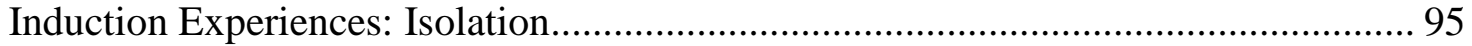

Induction Experiences: Weekly Reflective Journal Entry Isolation .......................... 95

Induction Experiences: PhotoVoice Experience Isolation......................................... 98

Summary of Isolation during Induction Experiences …………………………...... 98

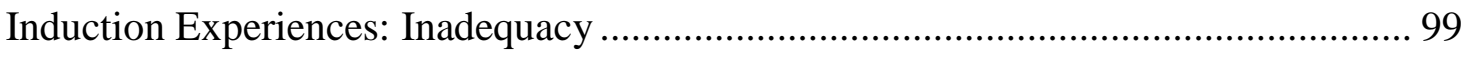

Induction Experiences: Weekly Reflective Journal Entry Inadequacy..................... 99

Summary of Inadequacy during Induction Experiences .......................................... 104

Closing Induction and Focus Group Session ............................................................. 104

Closing Focus Group: Compassion ................................................................... 105

Summary of Compassion in the Closing Focus Group............................................ 107

Closing Focus Group: Frustration...................................................................... 108

Summary of Frustrations in the Closing Focus Group .......................................... 110

Closing Focus Group: (from) Isolation to Tribe ………………………………....... 110

Summary of Closing Focus Group (from) Isolation to Tribe ................................... 111

Closing Focus Group: (from) Inadequacy to Self-Assurance.................................... 111

Summary of Closing Focus Group (from) Inadequacy to Self-Assurance .............. 113

Summary of Findings......................................................................................... 114

CHAPTER 5: SUMMARY AND DISCUSSION ……………………………......... 116

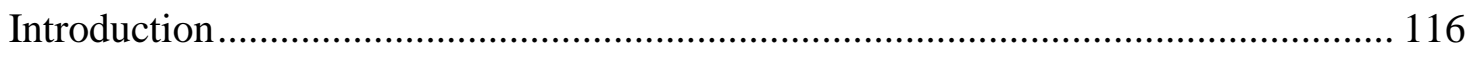

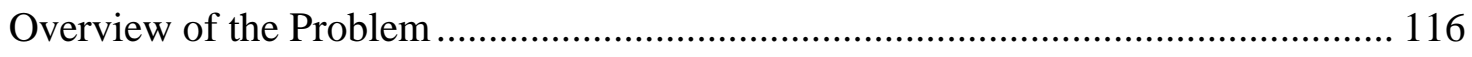

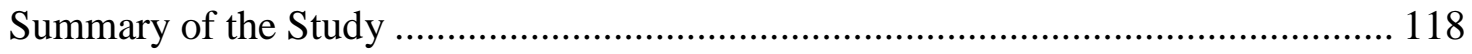



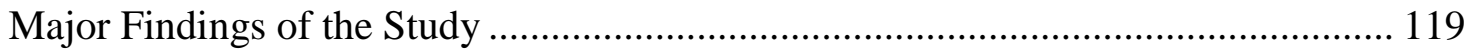

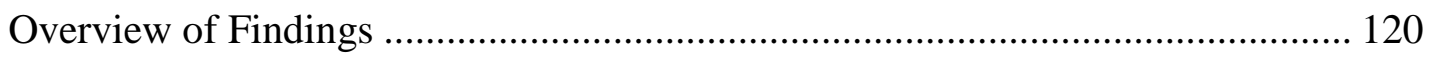

Conceptual Framework: Authentically Caring ..................................................... 120 
Conceptual Framework: Community Teacher................................................ 123

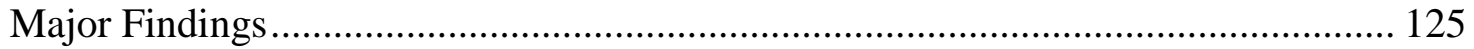

Major Finding Number One: Compassion........................................................... 125

Major Finding Number Two: Frustrations .................................................... 127

Major Finding Number Three: Isolation ...................................................... 128

Major Finding Number Four: Inadequacy .................................................... 131

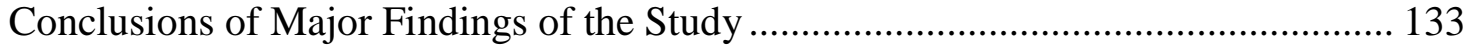

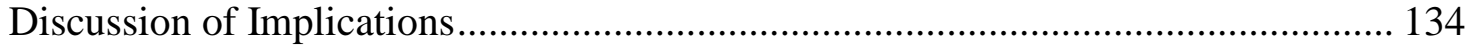

Implications for Policy Makers .................................................................. 135

Implications for Teacher Preparation Programs .............................................. 136

Implications for Urban School Districts .......................................................... 137

Implications for Prospective and New Urban Educators ................................... 139

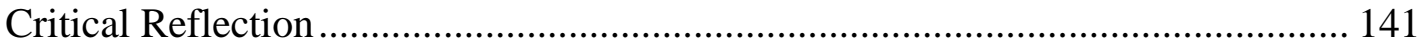

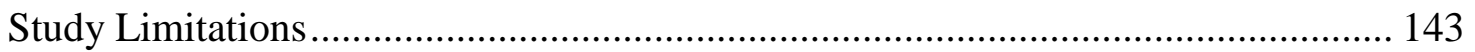

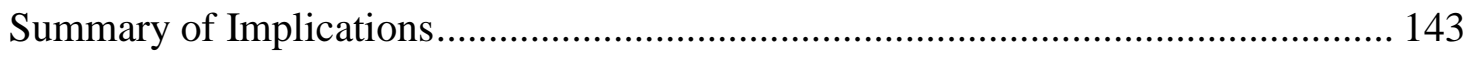

Recommendation for Future Research....................................................... 144

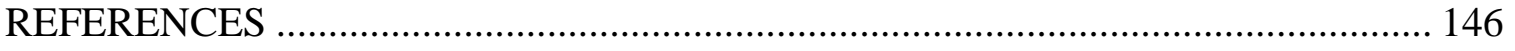

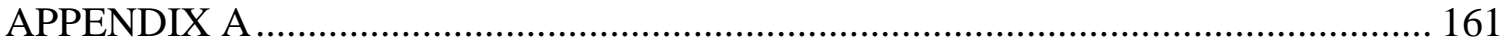

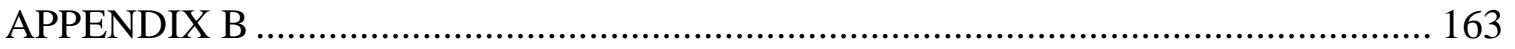

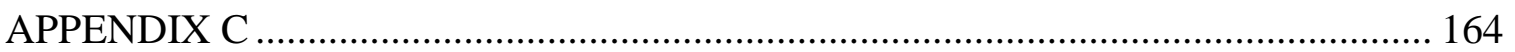

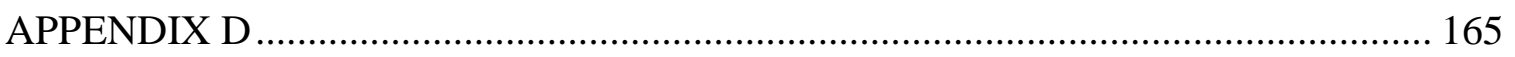

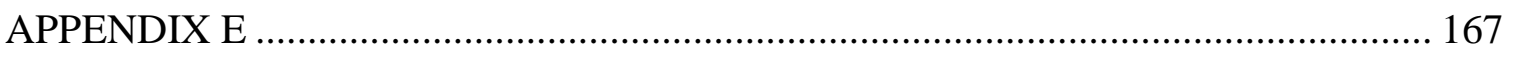

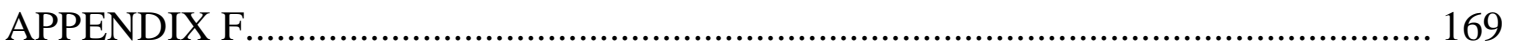

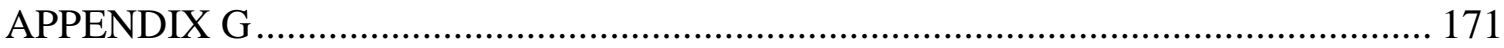

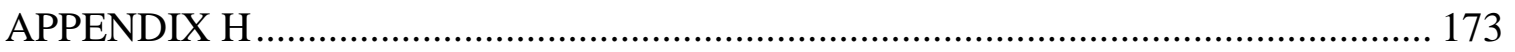

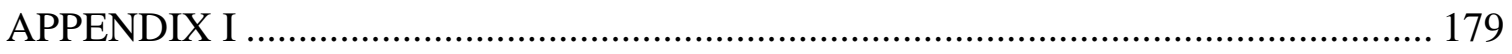

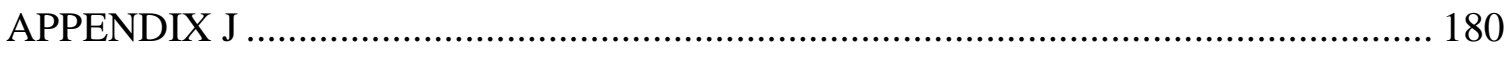

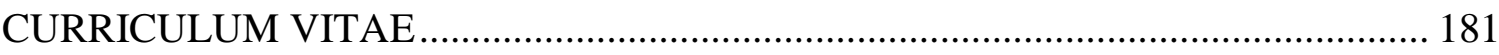




\section{LIST OF FIGURES}

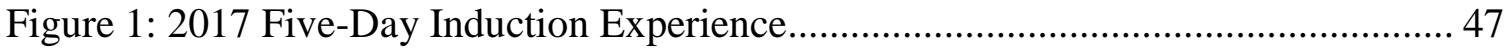

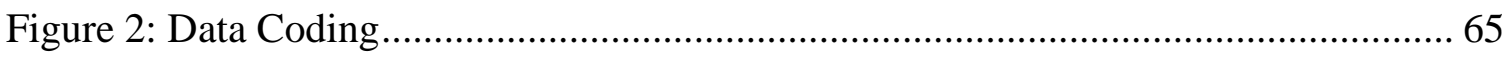

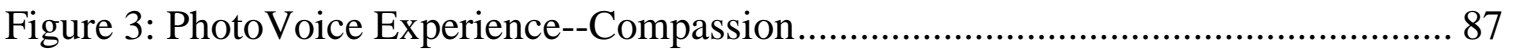

Figure 4: PhotoVoice Experience--Frustrations ............................................................ 93

Figure 5: Additional Reflective Thoughts from Participants ........................................ 114

Figure 6: Evolution of Themes Across Major Findings .............................. 133

Figure 7: Implication for Multiple Stakeholders ............................. 134 


\section{CHAPTER 1: INTRODUCTION}

Are there more than semantic differences between educators being ready to teach and being urban-ready to teach? Are there distinct differences between educators who graduate prepared to deliver best practice instruction to average public school students and those who possess those same qualities but also with an additional skillset that prepares them to meet the demands of our nation's most struggling urban schools? According to researchers like Haberman (1987, 2005), Murrell $(2000,2001)$ and Oakes, Franke, Quartz and Rogers (2002), there are specific skills or competencies beyond traditional pedagogical knowledge that educators who experience success in urban settings tend to display. This skillset extends beyond the general studies of most modern teacher preparation programs. Yet, there appears a lag in the willingness of some urbancentered universities to explicitly and intentionally seek to equip future teachers with these crucial add-on skills. In fact, the efforts of many promising programs designed to encourage success in urban teaching of the late twentieth century have been dismantled (Matus, 1999). I contend that there are indeed differences between ready and urbanready educators, and there is a need to intentionally equip future educators with those skills.

Some may question the logic behind the contention that there is a need to prepare urban educators differently than other practitioners. There are undeniable realities of urban communities that require educators to be otherwise informed and prepared to act 
appropriately based on an in-depth understanding of the complexities of surviving and thriving in urban environments. One such example is urban trauma and its prominence among urban children and their families. Abdul-Karim (2017) posits that these urban traumas occur as the result of disproportionate exposure to serious violence within the community and sometimes within the home. Additioinally, this researcher offers that these traumas are made worse by cultural biases related to unreliable access to quality physical and mental health care. These urban traumas manifest themselves as amplified emotional states, flashbacks, petulance and irritation, or rage aligned with the behaviors of post-traumatic stress disorders (Abdul-Karim, 2017). Supporting teachers-especially new teachers-in their efforts to teach students who have faced or are facing traumas is essential for the wellbeing of our most fragile population.

Further, developmental trauma disorder is the result of both chronic exposures to violent situations coupled with frequent disruption of primary caregivers in children (Stolbach et al., 2013). In their study, Stolbach et al. (2013) found a wide range of ways in which developmental trauma disorder adversely impacts children and categorized those ways as "affective and physiological dysregulation, attentional and behavioral dysregulation, self- and relational dysregulation, posttraumatic spectrum syndrome and functional impairments (Stolbach, et al., p. 6). These conditions that are the result of being on the downside of privileges — whether it is White privilege, religious privilege, two-parent home privilege or otherwise - undoubtedly influence the level of student readiness for learning.

In addition to disproportionate numbers of traumatized students, many urban schools may also face environmental challenges such as poor plumbing, limited 
instructional tools or supplies, and heavily taxed human resources. These schools are often positioned in economic and opportunity-deprived neighborhoods, making povertyrelated issues realities in the classroom as well. Not surprisingly, these schools often earn state exam scores condemned by the media for failing to meet minimal academic expectations, further perpetuating public perceptions of the hopelessness of urban populations (Morgan \& Kritsonic, 2008; Waxman \& Huang, 1996).

Camangian (2010) found that there are toxins plaguing urban communities that are impacted by economic development, politics, poverty, and limited resources. He went on to describe how these situational toxins create tensions within schools that manifest themselves as aggression and other harmful acts. Urban teachers must be prepared to strategically act and react with sensitivity to these and other urban actualities, remain mindful of the need for intentional self-care, and strive to meet academic expectations for students who need positively imagined and reimagined trajectories.

\section{Statement of the Problem}

"Hard-to-staff schools" (Morgan \& Kritsonic, 2008, p. 2) continue to present challenges for the public education system and, so far, efforts at addressing the issues have proven futile. Morgan and Kritsonic (2008) further explain that hard-to-staff schools exhibit characteristics such as a greater percentage of socioeconomically disadvantaged students, difficult teaching environments, school locations in areas some find less desirable, and low overall academic achievement within the student population. Helfeldt, Capraro, Capraro, Foster, and Carter (2009) found that one of the greatest challenges in education today is maintaining a highly qualified teacher presence and retention, especially in high-need urban schools. The features of these schools make hard-to-staff schools less desirable for teachers when other options exist. Still, these conditions persist 
in many high-need, urban schools across the country. In these schools where students frequently face challenges and traumas that would derail the personal and professional productivity of most adults, the realities of some urban children prove to be tremendous obstacles to the most eager of early educators to navigate.

\section{Teacher Shortage}

Given the state of conditions in high-need urban schools, urban school systems frequently find it challenging to fill classrooms with and maintain the presence of effective educators in their most struggling schools. While most teacher candidates graduating from teacher preparation programs do so with great enthusiasm, having successfully completed coursework, having passed teaching credential exams, and having been declared successful in field placements, these new teachers are often blindsided by the demands of teaching urban school populations (Green et al., 2011). Indeed, I have witnessed some teachers depart in revolving door fashion, surviving only days or weeks. The National Center for Education Statistics (2015) conducted a Beginning Teacher Longitudinal Study (BTLS) to explore patterns of early career teachers. The BTLS found that approximately 10 percent of the teachers who began their career in 2007 and 2008 were no longer teaching in 2008-09 and further report that in 2009-10, 12 percent were no longer teaching (Gray \& Taie, 2015). Others exit before reaching tenure status, spending five or fewer years in the classroom before abandoning the profession and their dreams of teaching (Ingersoll \& Smith, 2003; Nahal, 2010). I contend that those numbers are significantly higher in high-need urban schools. Ingersoll, Merrill, and Stuckey (2014) found that first-year teachers were leaving at an increasing rate, rising 34\%--from approximately 6,000 first-year teachers to about 25,000 between 1988 and 2008. The 
impact on students' learning may be predictable for some. The interruptions break consistency across schools and consume human and financial resources.

Additionally, when early career educators leave the school and/or abandon the profession altogether, classrooms filled with untapped student potential are orphaned and often thrown into chaos. Efforts to establish or reestablish a healthy learning environment often only yield a buffet of substitute teachers who simply attempt to hold order, as many are not certified educators and, therefore, not adequately equipped to advance student academic development. Additionally, schools do not receive the benefits of their monetary and professional investments in growth and development of the teachers who exited early in their careers (Donaldson, 2009). The cost of replacing even a novice teacher proves costly both economically and regarding effectiveness for schools that must invest in the recruitment and acclamation of replacements who must be given the latitude to grow into proficiency (Papay \& Johnson, 2012).

\section{Urban-Ready}

Effective urban educators must, in practice, build an urban-ready skillset to meet with success in urban schools. One may be led to question just what urban teachers must develop beyond the dispositions nurtured by traditional teacher preparation programs. Just what are the urban-ready, urban-competent or urban-effective teacher characteristics that allow some urban teachers to experience success in hard-to-staff urban schools? McKinney, Haberman, Stafford-Johnson, and Robinson (2008) codified 14 characteristics of teachers who tend to meet with success in urban schools based on Haberman's (2005) exploration of over forty years of research on the education of urban teachers. These urban educators are able to 1) guide the learning space, time, and opportunities, 2) be tenacious, 3) utilize strategies for success with at-risk students, 4) 
connect theory with practices, 5) professionally and personally orient themselves to students, 6) find the learning in their fallibility, 7) be emotionally and physically resilient, 8) be organized, 9) articulate teacher success, 10) articulate student success, 11) teach strategically and effectively, 12) establish a community where students feel they are a needed part, 13) carefully consider the material as it relates to students, and 14) teach with sensitivity to the realities of a sometimes violent society (McKinney, et al., 2008). These characteristics do not glamorize or make effective urban educators appear to be an exact science or path. To the contrary, they portray these educators as real, human, and flawed but fixed on a mission of stewardship. If these are the traits urban educators need, finding ways to nurture these traits may reduce urban teacher attrition.

In their quantitative study, McKinney, et al. (2008) found that urban-specific student internships alone were not significantly more likely to encourage future teachers to develop effective urban teacher characteristics than those who experienced traditional internships. They concluded that the short-term placement of student interns in urban Professional Development Schools (PDS) does not do enough to adequately prepare the interns to develop effective urban teacher characteristics. These researchers called for universities to do more to prepare urban educators for success in the classroom. I contend that the urban-specific internship may need to extend beyond the preparation program and into the classrooms of new urban teachers in high-need schools in the form of extensive induction experiences.

\section{Preparing Urban Teachers}

In another qualitative field-based study, Matus (1999) explored the impact on urban-specific coaching and seminars provided during student teaching that extended beyond the traditional structure of student teacher, cooperating teacher, and university 
supervisor triad. Matus' seven urban teachers found the urban-specific, yet evaluationfree support helpful and questioned why this thread of training and education had not been part of their on-campus work prior to student teaching. Many eager educators contend that their teacher education programs failed to prepare them for the demands of urban classrooms. Matus (1999) concluded that there was a need for urban-specific training as part of teacher preparation prior to student teaching and that university supervisors would benefit from specialized training in working with urban populations to better serve the student teachers they are supervising in those settings. Donaldson (2009) recommends that preparation programs assist new urban educators by fostering a commitment to the mission of urban education and providing future urban teachers with a realistic preview of the work prior to their decision to apply for, and certainly accept, a job assignment in the urban setting.

Ryan, Ensminger, Heineke, Kennedy, Prasse, and Smetana (2014) discuss the efforts of Loyola University as they worked to re-envision their teacher preparation program by linking their program both to the community and schools to nurture resilient and reflective educators. The goal of the updated program was to enrich the experiences of future teachers beyond the acquisition of credentials and employment in average schools. Instead, the new goal was to prepare educators with the finely honed knowledge, abilities, and dispositions that are effective when working specifically with urban communities through its children, families and schools. They explored eight key scopes of teacher education that include 1) approach, 2) framework, 3) research to practice, 4) stakeholders, 5) partners, 6) faculty roles, 7) teacher candidate growth, and 8) definition of success. Through an urban community-based refinement of each of these scopes, there 
was community, school system, and university investment in preparation of future urban educators. They crafted their list of 11 enduring understandings based on a backward design that included 1) social justice that seeks to empower vulnerable groups, 2) collaboration with varied stakeholders within and beyond the schools to encourage student success, 3) instruction that is aligned and designed to meet the needs of diverse students, 4) assessment of the effectiveness of both teaching and learning 5) policy understandings that allow for advocacy for students and families, 6) content knowledge and sound pedagogy for students with diverse needs, 7) diversity seen as an asset upon which to build with high expectations, 8) literacy explicitly positioned across learning contents and contexts, 9) the facilitation of safe and healthy environments, 10) theory informs decision-making, and 11) global perspective and mindfulness regarding student achievement. The program at Loyola and others like it are leaning on the teacher preparation programs across the country to be more responsive to needs of future educators and, in some ways, be more responsible for the success of these teachers.

I posit that, not in lieu of, but in addition to preservice student internships in urban Professional Development Schools (PDS), induction experiences within the first year in the classroom may serve to increase those effective urban teacher characteristics in new urban educators. Hoban (2005) argues the essential need for a theory-practice link in teacher preparation programs, yet there remains a gap between the work of these intentional programs in their theory and field practices for preservice teachers and the effectiveness of these new teachers when faced with the demands of urban classrooms in the weeks and months just beyond graduation and their placement in high-need schools. Exploring viable options for addressing the needs of new urban teachers must remain a 
priority for all stakeholders in public education if we are to make strides toward meeting the needs of our often most fragile students.

\section{Purpose of the Study}

Those invested in public education and education researchers have called for teacher preparation programs to do more to better prepare future educators for the work of high-need urban schools. Donaldson (2009) concluded that teacher preparation programs cannot and should not single-handedly shoulder the responsibility of preparing urban educators for the classroom. This researcher argues that principals, teacher leaders, and experienced teachers at the site level must adopt a tribe mentality in their determination to nurture young teachers as they develop their abilities to fully stand on their own, especially in challenging schools (Donaldson, 2009). He goes on to suggest a collaborative effort is likely to be effective "if educators at the school site and personnel from the preparation program work together to support new teachers" (Donaldson, 2009, p. 365).

In alignment with Ryan et al. (2014), Donaldson (2009), and the tribe mentality, I posit that the responsibility lies with multiple stakeholders. This study explores three instrumental stakeholders. First, any university positioned in an urban area and populating most of the public school classrooms in that school district with its teachers should bear significant responsibility for ensuring the urban-readiness and urbaneffectiveness of its graduates. In addition, the urban school district with a vested interest in the success of the teacher candidates they hire, and ultimately frequently place in highneed schools, must invest in these new teachers. Finally, teacher candidates seeking employment within an urban school system must do all they can to ensure their personal urban competencies (trauma-, poverty-, and culture-related) are as savvy, if not more 
savvy, than their traditional pedagogical skillset. Consequently, the university, school system, and teacher candidates must all approach urban classrooms and students strategically in order to begin addressing high teacher turnover rates if the goal is to truly yield more promising results with teacher retention and student academic and personal success (Gimbert, Desai, \& Kerka, 2010). This study explores all three sets of stakeholders.

To study collaborative stakeholder investment in new urban teachers that Ryan et al. (2014) discuss for preservice teachers and Donaldson (2009) suggests for new teachers, this study utilizes qualitative refractive phenomenological research (Abawi, 2012) to explore the essence of lived experiences of newly hired urban teachers participating in collaborative urban university and urban district provided induction experiences. This study also explores the participants' self-perceptions of their readiness for and effectiveness in urban classrooms during their first semester of teaching. This study adds to the research by providing a qualitative account of the lived experiences of new urban teachers who actively participate in collaborative induction experiences beyond an orientation-like phase that may take place prior to the start of the school year. Instead, this induction support is provided as new urban teachers establish themselves in their own classrooms for the first time.

The data sources utilized to explore the lived experiences of newly hired urban teachers engaged in this study include a researcher reflection journal, participant reflection journal, focus groups, and PhotoVoice (Mohammed \& Sajun, 2015). PhotoVoice is a participatory action research method that originated in China to give voice to marginalized populations (Maclean \& Woodward, 2013; Wang, Morrel-Samuels, 
Hutchinson, Bell, \& Pestronk, 2004). It is based on the belief that people are experts regarding their own story. Participants are charged with capturing images of their realities. They then use writing to provide context for the images and share them with the group to encourage reflective conversations. The group then codifies the collection of images and writings into themes. Sharing, discussing and reflecting on the images as a collective allows for a group approach for dealing with issues they may face (Wang et al., 2004). The use of PhotoVoice, reflective notebooks, and focus group data in a refractive phenomenology (Abawi, 2012) methodology allows for a deep analysis of the essences of the lived experiences of each of the study's participants.

\section{Conceptual Frameworks}

This study will be framed by Murrell's Community Teacher (CT) conceptual framework (Field \& Gutierrez, 2006; Murrell, 2000; Murrell, 2001). According to Murrell (2001), a community teacher is "one who possesses contextualized knowledge of the culture, community, and identity of the children and families he or she serves and draws on this knowledge to create the core teaching practices necessary for effectiveness in diverse settings" (p. 52). The CT framework was designed to address the need for situative collaboration between all stakeholders, increase the number of community teachers, and promote effective practices that meet the needs of the community it serves (Murrell, 2000). The CT framework presents a practice-oriented approach, in which community teachers feel called to give back to their community in meaningful ways, and actively work to transform the educational experiences of their students despite any inequities existing within the community (Murrell, 2000). Community teachers often live within their school community boundaries and are therefore more likely to be deeply 
invested in that community. Many community teachers may also be products of the education system in which they are employed (Murrell, 2000).

The study is also framed by Valenzuela's (1999) Authentically Caring (AC) framework. The essential elements of the AC framework are authentic connections, unwavering love, and a more complete or thorough understanding of others. The AC framework calls on teachers to view their relationships with students as connections that either subtract from or add to students' positive experiences in schools (Camangian, 2010; Valenzuela, 1999; Velliaris, 2016). Valenzuela (1999) argues, "Real learning is difficult to sustain in an atmosphere rife with mistrust" (p.7) and that vulnerability exists when educators engage only in aesthetic caring - caring linked primarily with instructional, non-personal components and, then, only as an afterthought if at all, the personal realities of students. Instead of aesthetic caring, AC calls on educators to draw and build upon the assets students bring to the learning contexts of school.

\section{Research Questions}

In an effort to explore the link between theory and practice for beginning urban educators who are provided collaborative, urban-specific induction experiences, I pose the following research questions:

1) What are the lived experiences of first-year urban teachers who participate in collaborative, urban-focused induction experiences?

2) What are first-year urban teachers' perceptions of the impact of the urbanfocused induction experiences on their self-efficacy as newly hired urban teachers? 


\section{Definition of Key Terms}

There are several key terms associated with this study that warrant definition. The following terms and definitions are presented to explain their use in the context of this research study.

High-need schools. This term is being used in reference to schools with a higher than average percentage of students living in poverty. The population includes higher than average number of students dealing with one or more adverse childhood experiences. High-need schools often also have greater diversity in the student population that may require additional support services to be prepared for school.

Newly hired urban teacher. This term represents teachers who have been hired for the first time by the large urban school district that is the setting of the research study.

Teacher resiliency. This term refers to a teacher's ability to calibrate and stabilize herself even when or just after facing adversity in the worksite. The resilient teacher continues to preserver in spite of the challenges that are sometimes inherent in high-need schools.

Teacher-student intersection of life experiences. This term refers to common prior life experiences that the teacher and student share. The term refers to shared significant life events or adverse childhood experiences that both the teacher and student have experienced that create a bond at personal, not necessarily academic levels.

Urban. This term has been defined in varying ways according to the context where it is used. For the purpose of this research study, the U.S. Census Bureau definition is being applied. An urban area is an area undergoing continuous development with a population of 50,000 or more and the urban clusters that surround that area (Johnson \& Johnson, 2015). 
Urban competence. Murrell (2006) and Oakes, Franke, Quartz, and Rogers (2002) have explored the tools and skillset required for educators to meet with success in highneed urban classrooms. For the purposes of this study, those competencies have been synthesized to include cultural competence, poverty-informed practices, knowledge of trauma-informed practices, and knowledge of the resources available within the community, and knowledge of the workings of neighborhoods as microcosms within the community.

Urban-focused induction. Induction experiences that extend beyond—not at the exclusion of - traditional induction experiences, urban-focused induction is designed to meet the needs of newly hired urban teachers. This induction includes experiences designed to nurture urban competencies in educators.

Urban-ready educators. Educators who not only possess sound pedagogical and other traditional essential teaching attributes, but who also have developed and continue to nurture those skills that increase their ability to connect with and successfully teach students from traditionally disenfranchised, disproportionately traumatized, more challenging populations are urban-ready. For the purposes of this study and beyond, this term remains fluid and will evolve as the attributes that garner success in urban schools continue to evolve.

Urban schools. For the purposes of this study, urban refers to the inner-city of a large, diverse city. The focal urban city of this study featured a population of over 616,000 in 2016 with a public school system of near 100,000 students. The urban schools of focus for this study are those servicing diverse, high-need students and are often hard to staff with experienced teachers. 


\section{Summary of Chapter}

In this first chapter, I have provided context for the challenges large urban school districts face with regard to hiring and maintaining teachers who are prepared to successfully lead the learning lives of students in high-need urban schools. This chapter highlights the call for teacher preparation programs to focus on preparing teacher candidates for today's urban schools. It also outlines the need for collaborative urban induction experiences that involve multiple stakeholders in an investment in new urban teacher success. In this study, I explored the roles occupied by the local urban university, local school district, and newly hired urban teachers themselves. Additionally, key terms have been defined to provide context for readers.

Chapter two presents a landscape of the literature found on the topic of urban teacher induction and urban teaching in general. The chapter positions the study within the context of the conceptual frameworks, Authentic Caring (Valenzuela, 1999) and community teaching (Murrell, 2010). The chapter more closely explores cultural competence, poverty-informed practices, and community-based teaching as instrumental skills for competent urban educators. 


\section{CHAPTER 2: LITERATURE REVIEW}

\section{Introduction}

In this research study, I explored the lived experiences of first-year urban teachers who participated in collaborative induction experiences with the support of multiple stakeholders. This study adds to the literature on the experiences of first-year teachers in urban schools and classrooms, informing the design of induction programs that work to mitigate teacher attrition. The study included the following research questions

1) What are the lived experiences of first-year urban teachers who participate in collaborative, urban-focused induction experiences?

2) What are first-year urban teachers' perceptions of the impact of their lived experiences in the urban-focused induction experience on their self-efficacy during the first semester of teaching?

In this review of the literature, I explore the challenges facing urban education in modern times, specific factors associated with urban teacher attrition, and the characteristics linked with urban teacher competencies that serve as pillars in this research study. This following section explores literature regarding the trials of urban education.

\section{Urban Educational Challenges}

There are challenges that public education in urban areas seem to accept as inherent in the landscape due to larger, persistent systemic problems, such as issues viewed as consequences of poverty, the increasingly diverse needs of increasingly diverse 
students, elevated principal turnover rates, and teacher attrition. With deeply rooted systemic issues plaguing public education, researchers explore solutions for addressing aspects of the array of challenges to move us closer to an educational system that meets the needs of the diverse population of urban schools. In many of these schools, there are students who have basic human needs that go unfulfilled on a regular basis. This population often includes a disproportionate number of students who have experienced or who are currently experiencing one or multiple life traumas commonly known as adverse childhood experiences or ACEs such as exposure of violence, varying forms of abuse, and illnesses (Bethell, Newacheck, Hawes, \& Halfon, 2014; Forster, Gower, McMorris \& Borowsky, 2017; Hunt, Slack \& Berger, 2017; Metzler, Merrick, Klevens, Ports, \& Ford, 2017; Wade, Shea, Rubin, \& Wood, 2014). The ACEs have an impact on the most eager and ambitious student to cross the threshold of the classroom on a daily basis. The results of the baggage these students carry are multifold and taxing on urban schools and, especially, the new urban teachers who often fill the urban school classrooms.

Large urban school districts are growing more diverse all the time (Fayne \& Matthews, 2010; Proctor, Rentz, \& Jackson, 2001; Smith \& Ingersoll, 2004). These school districts are often faced with a wide range of barriers to learning. Urban schools are populated with our most fragile students. These students require more of teachers than best instructional practices that are traditionally part of preparation programs (Dorman, 2012; Duck, 2007; Onore \& Gildin, 2010; Proctor, Rentz \& Jackson, 2001). Some are experiencing traumas that threaten their physical safety, and they exist in survival mode. There are also students who are starved for the social-emotional bonds that traditional 
families often offer. These students need schools and their teachers to care for more than their academic needs (Dods, 2013; Waxman \& Huang, 1996).

\section{Adverse Childhood Experiences}

According to Bell, Limber, and Robinson (2013), childhood trauma negatively impacts multiple aspects of the child's school experiences, including relationships with peers, overall communication skills, and, certainly, academic achievement. Sitler (2009) argued that traumatic life events overwhelm one's sense of meaning, connection, and control. For example, Martin (1991) found that school-aged children who suffer the trauma of homelessness, if able to attend school, then face the added trauma of moving from school to school and trying to become a member of already cohesive classrooms filled with often reluctant teachers who brace for challenging behaviors from the potentially traumatized student. This convoluted web leads to students suffering from academic challenges due to inconsistencies in instruction and home environments not conducive to successful academic lives. These conditions fuel sweltering rage, withdrawal, and depression in students who tend to exist in greater numbers in hard-tostaff schools.

There is a need for schools and teachers to provide safe spaces for students dealing with varying levels of trauma. Bell, Limberg, and Robinson (2013) describe two kinds of trauma. Type I traumas or acute traumas, are the result of a single event like natural disasters, terrorist attacks, or the loss of a loved one and are usually short-lived. Type II or chronic traumas are characterized as traumatic experiences that last over long periods of time and include abuse, neglect, chronic illnesses, and domestic violence (Bell, Limberg \& Robinson, 2013). Fostering resilience in traumatized students is essential to ensuring fragile students have an opportunity to find meaningful existence and purpose 
beyond circumstances (Waxman \& Huang, 1996). In their quantitative study of 75 resilient students (scoring at or above the $90^{\text {th }}$ percentile on math achievement tests) and 75 non-resilient students (scoring at or below the $10^{\text {th }}$ percentile on math achievement tests) in a large metropolitan area, Waxman and Huang found in their discriminant function analysis that factors such as motivation to achieve, clarity of rules, self-concept in social settings, task orientation, and satisfaction were most discriminant. This indicates that non-resilient students may not be intrinsically motivated to perform as traditional school encourages. Meeting with success in urban settings may require teachers to be able to nurture resilience in students who may feel polarized by their life circumstances.

While ACEs include issues associated with poverty, such as hunger and inadequate health care, there are also students who are starved for the social-emotional bonds that are often inherent in traditional families. The collateral damage of these ACEs manifest themselves in ways that tax seasoned educators in experienced schools. Let us imagine the impact on new educators who may not be aware of the prevalence of ACEs in urban schools, and in some cases, the number of students in their care for several hours a day who are living with ACEs. These students demand attention and supports from their schools, and their teachers, beyond those covered in traditional teacher preparation pedagogy. Traditional teacher preparation programs simply do not prepare teachers to meet the needs of these students. This qualitative study contributes research beyond existing works that acknowledge the need for a multi-stakeholder investment in new urban educators to positively impact their work with high-need urban student populations. 


\section{Poverty and Diversity of Students}

When one sets out to explore the challenges of urban education, it is difficult to find studies that speak exclusively about poverty without a diversity variable. Most studies explore challenges through the lenses of both those that are related to poverty and those related to meeting the needs of such a diverse population of students. Likewise, many studies that explore race and diversity rarely exclude factors related to poverty. In urban contexts, few would dispute that connections exist between studies of students living in poverty and studies of Black and Brown students. Milner and Laughter (2015), in a qualitative study, explored race and poverty independently but also the intersection of the two. Through the lens of critical race theory (Ladson-Billings, 1999), they set out to learn more about how policy and practice in teacher education may be reformed to ensure that students of poverty and those Black and Brown students increase their chances at success. These researchers noted that there is no certainty that Black and Brown teachers can effectively teach Black and Brown students, just as there are no automatic exclusionary factors that prove that a White teacher cannot teach these same students. For this reason, there is room for all teachers to engage in discourse around race, poverty, and the intersection of the two. Milner and Laughter (2015) also suggest that teachers puzzle through questions like "Does poverty manifest for White students in the same way as for Black students? How are these emergent manifestations similar and different? What is essential to understand about these similarities and differences as educators teaching in the public schools?" (p.344). While questions regarding race and poverty can be uncomfortable, these researchers are challenging educators to be comfortably uncomfortable as they brave tough conversations and reflections about race and poverty and the influences of each in school settings. 
It has been argued that money only really matters when you do not have enough to meet the basic needs of self and family; when money, or the lack thereof, is the obstacle that consumes your ability to think beyond the immediacy of meeting those basic needs, it matters. Many students and families within inner cities or urban communities face economic challenges that have an impact on student learning in educational environments (Milner \& Laughter, 2015; Ullucci \& Howard, 2015). Jensen (2009) defines poverty as "a chronic and debilitating condition that results from multiple adverse synergistic risk factors and affects the mind, body, and soul" (p. 6). Jensen's research also led him to classify poverty into these six categories, though not at all mutually exclusive, by context: 1) situational or temporary poverty that occurs after an often unexpected crisis, 2) generational poverty which exists over two or more generations within a family structure, 3) absolute poverty which occurs when there is a scarcity of basic day to day survival needs such as food, shelter, and clean running water, 4) relative poverty which occurs when a family's income fails to sufficiently accommodate average needs within society, 5) urban poverty occurs within areas of more than 50,000 citizens where overcrowding, noise pollution, inadequate services, and violence are commonplace, and 6) rural poverty within areas of fewer than 50,000 residents where the availability of viable employment, adequate resources are obstacles, as are single-parent households (Jensen, 2009). He sternly reminds us that poverty is not absolute, meaning different things to different people, but, within school contexts, poverty tends to manifests itself in higher incidences of inappropriate and disrespectful behavior, as well as frequently missed instructional time due to tardiness and diminished motivation. Those living in poverty are also disproportionately represented in the students 
who enter school settings linguistically deficient and low on exposure to non-academic learning experiences such as the theater, museums, and educationally rich vacations (Cuthrell, Stapleton \& Ledford, 2009).

Many researchers have conducted studies to examine ways educators - from teacher preparation programs to school districts and teachers themselves - may fill the gaps created when the urgencies, traumas, and weight of poverty overshadow the significance of educations for students who would benefit most. Ullucci and Howard (2015) presented their work on poverty in hopes that they could change the narrative on educating students of poverty from a deficit mindset perspective to one that acknowledges both the damages and promise of educating economically disadvantaged students. They explored mythologies related to poverty that impact student-teacher relationships and examined new perspectives for educating the impoverished child through the use of guiding questions to help teacher educators explore issues of poverty with preservice teachers. In their work, they posit that the impact of poverty on students is real and far-reaching, yet they challenge generalized beliefs that economic challenges directly correlate with problematic or unsuccessful academic outcomes. They note the resourcefulness, resilience, maturity, and leadership many of these students exhibit in spite of the living conditions of these students as well. They caution educators to avoid hyper-generalization of how poverty impacts students. Hyper-generalization may lead to victim blaming as these teachers tend to believe their teaching has little if any impact of student academic advancement. They suggest that a comprehensive, critical framework for understanding poverty must be approached without blaming students and their families. Instead, they suggest acknowledging the economic, social, and political 
conditions that impact these communities. Ullucci and Howard (2015) offer the following questions for thinking through this framework.

1. What are the systemic and institutional factors that impact poverty?

2. How does poverty impact students' lives?

3. What assets reside in low-income communities?

4. Do schools provide equal educations to children in poverty?

5. Can school eliminate poverty?

The researchers admit the density of puzzling through these questions but posit that work like this is necessary. They suggest that, while there are factors of poverty that schools cannot influence, the heavy-lifting of unpacking the factors that we can influence cannot be confined to a single class session within a broader class on urban education for preservice teachers. It is essential that teaching be context-specific as opposed to context neutral. Educators must avoid distancing themselves in egocentric ways that involve the othering of people living in poverty by illuminating self-perceptions of how we know better, do better, and are better than those we serve in gestures of gross marginalization.

Teachers must develop a deeper understanding of the implications of poverty on the lives of the students they teach. Researchers like Ullucci and Howard (2015) examined the work of teacher educators regarding students of poverty. They reasoned that it is critical for teacher preparation programs to be mindful of how they prepare future educators for teaching impoverished students.

Hammerness and Matsko (2013) used a case study design to examine the induction of new teachers who were part of the University of Chicago's urban teacher education program by intentionally considering the context of the specific school and 
students of the urban setting as the content for teacher learning. The contexts explored in the study included educational policy context, urban public school context, local geographical context, local socio-cultural context and children, classroom, and school context. These researchers utilized document analysis, an interview of the director of the urban education program, and a focus group with faculty and staff to explore what induction looks like when it takes context into consideration. They found that contextspecific support encouraged the development of new teachers' knowledge regarding economic, geographic, and cultural attributes of the district as well as local school knowledge of specific school curriculum, routines, and procedures. They found that context was the content for teaching and learning for the program's participants and suggest that this explicit, specific support may encourage increased longevity within the profession of education as well as potentially fostering retention within the school. Gaining context-specific knowledge increases an educator's ability to meet the specific needs of the population (Hammerness \& Matsko, 2013). An understanding of the context at the school level can be facilitated and nurtured by the school administration and other stakeholders.

As a result of their analysis of current practices, Milner and Laughter (2015) developed three policy recommendations. The first is a call to reform teacher education curriculum to emphasize race, including a deep understanding of what it is and how it is defined, conceptualized and constructed physically (skin color), socially (societal constructs), legally (landmark court cases), and historically (past treatment). The second reform policy recommendation calls for the reform of teacher education curriculum to emphasize the study of poverty. Milner and Laughter (2015) purport that poverty has a 
lingering effect on the social, psychological, and economic well-being of people.

Teachers would benefit from a more informed perspective of students living in poverty. Their third policy recommendation is for the reformation of teacher education curriculum to explore the nexus between race and poverty. Teachers would benefit from understanding how the number of Black and Brown people living in poverty is attributable to discrimination and racism and other marginalizing actions.

\section{Factors Associated with Urban Teacher Attrition}

Capraro, Capraro, and Helfeldt (2010) draw upon the work of Gless and Barron (1992) to assert that new teachers tend to transition through five phases during their first year in the classroom. Those phases include (a) anticipation, (b) survival/disillusionment, (c) rejuvenation, (d) reflection, and (e) anticipation of the subsequent school year. Many urban school districts are experiencing a mass exodus of teachers shortly after their entry into classrooms. While the anticipation phase brings great hope and excitement for the potential to have a lasting impact on the lives of children, the survival and disillusionment phase is where many new urban educators realize the magnitude of the job and are, in fact, not surviving. New educators often quickly become overwhelmed by the enormity of what they do not yet know and are not yet able to do well. Those in our urban high-need schools are often unable and/or unwilling to allow themselves to gain the experience needed to succeed. Instead, they often flee, leaving underserved classrooms. Especially in high-need schools, this often means high-quality instructional triage is critical for stabilizing often sinking academic ground.

Ingersoll and Smith (2004) found that many teachers vacate the profession after three to five years in the field. The challenges are exacerbated in urban settings. Urban school systems frequently find it challenging to staff their classrooms with certified 
educators in their most struggling schools. Those once enthusiastic, starry-eyed teachers find themselves completely overwhelmed by the realities of challenging urban classrooms (Green et al., 2011). In fact, I have witnessed those who resigned after only weeks or days. Others exit quickly, spending only a few years in the classroom before abandoning their teaching career (Ingersoll \& Smith, 2003; Nahal, 2010). Classrooms in hard-to-staff schools find themselves scrambling to recover when teachers have accepted the position only to abandon it without completing the year. The goal and intent of educating students is replaced by the schools' efforts to provide coverage by often uncertified substitute teachers or other uncertified personnel. Hard-to-staff schools continue to challenge the public education system and, so far, efforts at addressing the issue have not been able to help us avoid teacher shortages. According to Helfeldt et al. (2009), “preparing and retaining highly qualified teachers for our nation's schools is one of the greatest challenges facing American education," (p.1) especially in high-need urban schools. Hard-to-staff schools are less desirable for teachers when other options exist, yet these conditions persist in many high need, urban schools across the country. Given this picture of urban education, retaining urban teachers is a challenge that makes a great deal of sense, yet researchers are still looking for ways to better understand teacher attrition and to find viable solutions to retaining teachers who remain in the classroom long enough to reach a level of proficiency that translates to improvements in student performance.

Few would argue the challenges inherent when teachers do not remain in the profession long enough to reach professional maturity. There are times when the attrition of a teacher is what is best for students, the teacher, and school. In these instances, their 
retention would be detrimental to all parties. These organic instances are warranted if an ineffective teacher is holding on when unhealthy conditions exist and persist. However, when teachers depart hard-to-staff schools, they leave in their wake gaps that are difficult to fill. Brill and McCartney (2008) found that along with economic, institutional, and instructional costs, high teacher attrition is especially hard on low income and high minority population schools. The economic costs appraised by researchers vary, but Brill and McCartney (2008) estimate the range of 20 to 150 percent of a teacher's salary. This equates to between $\$ 2.2$ billion and $\$ 4.9$ billion dollars annually to find, recruit, hire, and train new teachers. The institutional costs also include those of community and collaboration. Constant or frequent disruptions in the school teams impact the school's ability to develop and present a unified, cohesive, and comprehensive curriculum. These researchers further claim that the impact hits twice as the money that was spent to train exiting teachers cannot benefit the students they leave behind, and there is the cost of training those replacements which also breaks cohesion. The instructional costs are realized by the idea that more experienced teachers are usually more effective with students than new or newer teachers (Brill \& McCartney, 2008). When thinking about conditions that high attrition presents to low-income and minority schools, Prince (2002) shared the following words from one administrator in her study:

I can't help where my school is. No matter what I do, I can't keep a staff in here. I'm lucky if I have a breathing body for every classroom, and the ones I end up with are the very bottom of the barrel. I'm not only in a bad part of the city, I'm in a school with a bad reputation in a bad part of the city... When I mention staffing they tell me I'm whining again. (p. 13) 
These administrators are working in conditions where the attrition rate was twice as high in other schools. This proves especially impactful on the learning of a fragile population who would benefit from the most experienced and successful urban educators. Brill and McCartney (2008) outlined the challenges presented when teachers choose to terminate their service at the classroom level.

Researchers are also looking more closely at the specific reasons teachers are exiting the classroom and have found those reasons to be varied in scope and nature. In teacher attrition literature, teachers are categorized as leavers, movers, and stayers (Freedman \& Appleman, 2009; Kukla-Acevedo, 2009; Olsen \& Anderson, 2007). Leavers are those who quit the profession. Movers either simply change schools (usually moving to a school with higher-performing students) or take on roles outside of the classroom but remain in the education field. Stayers are those who remain in the school where they began teaching. Kukla-Acevedo (2009) explored the mobility of 3,505 teachers in their first five years of teaching in a binomial and multinomial exploration of three workplace conditions - school climate, autonomy in the classroom, and administrative support. This researcher found that two of the three predicted conditions had a statistically significant impact on novice teacher mobility. Those two conditions are administrative support and the climate of the school. Surprising the researcher, autonomy did not have a statistically significant impact on novice teacher mobility. Kukla-Acevedo (2009) calls for additional exploration of both the movers and leavers to differentiate the response efforts to ensure high-quality instruction in classrooms.

Olsen and Anderson (2007) conducted a qualitative study of 15 novice teachers, those with six years or less in the profession. The study was designed to provide insight 
into why these teachers entered the profession, where they taught, why and how long they taught there, what compelled them to stay or leave their particular schools and/or the profession altogether, and what factors influenced highly qualified teachers to remain in schools of great need. At the conclusion of the study, they found that six of the fifteen stayed, six were uncertain, and only three left the profession. These researchers caution us not to bemoan leavers as their reasons sometimes include shifting to other positions within education that are still closely linked to their decisions to enter the field in the first place. They also found that the university had groomed teachers from the start to see education beyond the classroom perspective which may have contributed to the shifters. While there was a common desire among their participants for meaningful on-site mentorship and opportunities to interact with more experienced teachers, these teachers were mostly committed to the weighty work of urban teaching and saw themselves connected to the cause both inside and outside of the classroom.

In their review of the literature, Brill and McCartney (2008) found that the varied reasons for departures include class size and workload, student behavior, leadership and administration, facilities and resources, maternity, those who see teaching as a temporary profession, and poorly executed induction and mentoring programs. While they found teachers prefer smaller class sizes, few mention class sizes as a reason for their departure. Student behavior has proven a factor that influences teacher retention, cited as the second most common reason for leaving behind the most common reason—-teaching workload. Brill and McCartney (2008) present findings that align with Feng (2010) in his quantitative study that shows new teachers are often placed in the most unruly classrooms within hard-to-staff schools. Feng (2010) stated, "Within schools, there were differences 
in the kind of classrooms that inexperienced teachers were assigned. They were more likely to teach in classrooms with a larger share of low-performing, unruly students and students from disadvantaged backgrounds" (p. 312). These conditions produce stress for those new to the profession who have not developed the skillset necessary to manage some learning environments. Brill and McCartney (2008) also align with Waddell's 2010 qualitative look at teacher retention and asserts that the leadership and administration were reported as influencing teachers' decisions to stay or leave. Waddell (2010) found that three external influences associated with teacher retention. They include relationships with co-workers, students, and principals. Notably, the administratorteacher relationship and the provision of support to the teachers were identified as vital to teacher retention. Those who see education as a professional stop along the way also was described as a factor of retention — perhaps along the way to parenthood and therefore less than full-time work or a career switch. Finally, Brill and McCartney (2008) reason that ineffective and poorly orchestrated induction and mentoring programs cause more harm than benefit to new teachers. These studies seem to paint a cautionary picture for those invested in the retention of proficient teachers.

Encouraging new teachers to stay in the classroom to reach professional maturity or proficiency has been the focus of researchers. Relationships with students, co-workers and principals, self-efficacy, service, perseverance, and a sense of ownership are all factors Waddell (2010) found to encourage retention in her study. Freedman and Appleman (2009), in their mixed methods study, found six factors that influenced teachers to remain in high-poverty urban school. These were: 1) a sense of mission, 2) persistence and a disposition for hard work, 3) depth of academic and practical 
preparation for the work, 4) a reflective teacher-research stance or approach to the work, 5) opportunities for shifts within the profession and 6) ongoing support within a cohort and/or other teacher professional networks. Brill and McCartney (2008) found the literature to call for salary increases and incentives, professional development and a work environment that includes professional learning communities, and induction and mentoring programs as viable ways to address teacher attrition. All of these researchers encourage the education field to not see retention as a lost cause but as one that, if strategically and systematically addressed, will yield results that are more favorable for students.

Specifically related to my research study, Brill and McCartney (2008) call for an induction experience that includes preparing, orienting, and aiding new teachers in their actual practices. My study supports the transition of new teachers through this recommended process by providing a bridging experience from teacher preparation into the classroom. By involving those who prepare teachers (teacher preparation programs), those who orient new teachers (the hiring district), and the new urban teacher, my study sets out to provide the support needed for new teachers to meet with success in their actual practices once they assume the leadership of their own classrooms. While mentoring and induction carried out haphazardly or in overly aggressive ways encourage departure, mentorship and induction orchestrated effectively shows potential to influences new urban teachers' decisions to remain. The collaborative, field-based induction experience approach taken in this study seeks to facilitate conditions for early classroom success in hopes that early success will encourage more stayers to embrace the fulfillment of classroom teaching. 


\section{Secondary Trauma}

According to a growing body of research, secondary trauma is another cause attributed to teacher attrition. The research on childhood trauma positions children as products of their environment and experiences. The need for mental health care in schools is gaining popularity in urban school districts, including the district of focus for this study where leadership recently announced plans to include mental health counselors in every school. Just as attending to the needs of traumatized students is becoming a greater focus, some are noting the impact of caring for these students on those who teach and otherwise work with them. Researchers like Bride, Radey and Figley (2007), Sharp Donahoo, Siegrist and Garrett-Wright (2017), Ledoux (2015), Matus (1999), and Showalter (2010) are studying secondary trauma, compassion fatigue, or vicarious traumatization found in educators and other helping professions who work with traumatized populations. Bride et al. (2007) explain the connected terms in this way. It is now widely recognized that the indirect exposure to trauma involves an inherent risk of significant emotional, cognitive, and behavioral changes in the clinician. This phenomenon, variously referred to as vicarious traumatization (VT), secondary traumatic stress (STS), and compassion fatigue (CF), is now viewed as an occupational hazard of clinical work that addresses psychological trauma; a view supported by a growing body of empirical research (p. 155).

They have found evidence of educator exhaustion or fatigue related to an inability to separate the workings of educating children from the traumatic experiences of some children. Some researchers point to stark contrasts between novice middle-class teachers and the problematic conditions they face in some urban setting. 
Many of those novice teachers, who lack adequate urban teacher preparation, may be overwhelmed by the problems associated with urban teaching. The problems they will face, such as students' antagonism toward school, their lack of respect for themselves and their teachers, poor student discipline, high rates of student absenteeism, students with poor academic skills, large classes, and shortages of books and supplies—along with gangs, violence, drugs, weapons—can be traumatic for middle-class teachers who have had no experience with or preparation for working with poor, inner-city teenagers. (Matus, 1999, p. 37)

Such perceptions and realities persist and are often given wide-spread coverage by media outlets. In turn, this gives rise to greater negative connotations of teaching today for those exploring career options. This may discourage some people from considering the education profession or encourage those who choose the field to go to great lengths to avoid working with certain populations (Jacob, 2007). As a result, students and schools who need the best supports often do not receive it, thereby fanning already challenging flames. Closely linked to secondary trauma is compassion fatigue.

\section{Compassion Fatigue}

Some departures may be attributed to compassion fatigue. There is growing research on compassion fatigue (Bride, Radey, \& Figley, 2007; Sharp Donahoo, Siegrist, Garrett-Wright, 2017; Ledoux, 2015; Showalter, 2010). Compassion fatigue is found in many helping professions, such as health care and education. Showalter (2010) explains, Many of us in the helping professions are secondary witnesses to trauma on a regular basis. As witnesses and healers, we cannot help taking on the emotional pain of those we are privileged to know and care for. We collect bits and pieces of their trauma by exposure to their lives. We have pictures in our minds and intense 
feelings in our bodies at the end of the day. Those who are strongly empathetic may be most at risk (p. 239).

Compassion fatigue and secondary trauma lend explanation to the stresses those in helping professions, like education, carry as a possible consequence of the work. The negative impact of close work with people who have been traumatized in various ways may lead some educators to abandon the profession.

We are presented with the challenge of occupying our most fragile schools with teachers who are urban-ready - teachers equipped to effectively meet the needs of urban schools and student populations in today's educational landscape (Oakes, et al., 2002). Without changes to current systems, the near crisis status plaguing the teaching profession will continue to perpetuate a revolving door of eager educators who enter but abruptly abandon the profession. For these reasons, a closer look at the urban teacher, school and district characteristics, and new teacher supports are warranted (LadsonBillings, 1999).

\section{Conceptual Framework: Community Teacher}

Defining just what effective urban teachers need to meet with success in urban classrooms remains fluid as the needs of the student population evolve. Gimbert, Desai, and Kerka (2010) support Murrell's (2000) CT framework as they discuss a framework that includes community teachers and communities of practice where teachers work closely with and deepen their knowledge of the communities they serve, all to the advantage of students. They contend that this connection is critical to the development of successful urban teachers. Murrell's (2000) CT framework is multilayered-1) at the micro level of addressing the needs of individual learners, 2) at the meso level of the school community to nurture relationships in cultural, professional and social ways, and 
3) at the macro level to address the development of family, neighborhood, and institutional supports. He goes on to describe the community teacher as an urban educator with multicultural competence who may live in the community they serve, may be products of the school system they now serve, and may have had prior success working with youth within that community in some capacity. These community teachers view themselves as change agents and education as a critical key to success. Murrell (2001) goes on to define the community as a teacher who "develops the contextualized knowledge of the culture, community, and identity of children and their families as the core of their teaching practice" (p. 340). The community teacher is fully invested in and driven to have a positive impact on the community they serve. They are urban-ready as they see their connections with the community as essential to their success.

\section{Conceptual Framework: Authentic Teaching}

The authentic caring (AC) conceptual frame of Valenzuela (1999) contends that schools are primed in a position to either add to or subtract from the educational experiences of students. Anchored in work with Mexican-American students, she contends that teachers tend to expect students to care about school before the teachers care for them as people, yet students expect their teachers to care for them as people before they care about the academics of school. Valenzuela describes subtractive schooling as the position of the school to either add a second language and culture to the Mexican-American students or subtracting from their original language and culture. She contends that to suppress the original language and culture of a student is to subtract from their experiences of schooling. Instead, this researcher suggests authentic caring for the child's entire well-being by the teachers and other school adults. The authentically caring teacher and school care for the child first and care about the schooling next. The caring 
position has great influence over the students' perception of their ability to achieve at higher levels in school and beyond. Without authentic caring, she contends that these students may feel they are simply objects to be tolerated in schools (Valenzuela, 1999; Valenzuela \& Rubio, 2018). An authentically caring, urban-ready teacher and school environment create conditions that enable students to thrive.

\section{Urban Teacher Competency}

New teachers entering the field of education may have premature expectations that they will be hired to teach in classrooms that mirror their K-12 learning environments. The reality in urban communities is that many new teachers are hired to teach in the schools of greatest need, the schools that are proving hard to staff in the district. Urban competency was explored by Oakes et al. (2002) who posit that urban teachers need competence in understanding the urban cultures, the political economy within that urban culture, the organizational structure of urban schools, the community and social services systems in place to support the community, strategies for building on the social capital found within the community, and the ability to establish and grow alliances within the urban community. These researchers contend that effective urban teachers must be a total package of sorts — skilled both in quality classroom practices and committed to advocacy for democratic participation, equity, or access for students. The teachers are accountable to one another, their students, and the community they serve and work collaboratively for the common good. Context-specific learning is crucial to the development of urban competencies. These educators establish a sense of mutual trust between the communities they serve and the educator and, ideally, the educational institution. Oakes et al. (2002) called for deeper exploration of support for new urban 
teachers that connect schools, communities, and teachers as a strategy to increase teacher commitment to high-need urban schools.

\section{Urban Teacher Preparation}

Many researchers have found that while teacher preparation programs are aware of the need to make ready their teachers for the challenges and rewards of teaching in high-need urban schools, many fail to gauge preservice teachers' urban commitment prior to and throughout their teacher preparation experiences (Epstein, 2005). Taylor and Frankenberg (2009) posit that universities should help to ensure that aspiring teachers are self-aware of their urban commitment prior to entering the education field. This would aid them in gaining employment in a best-fit setting, and, ultimately, increase teacher retention. To illustrate, many teachers and teacher candidates fear and actively seek to avoid the unfamiliar. Taylor and Frankenberg (2009) found that newly hired teachers or teacher candidates feel uncomfortable teaching in environments that are unlike their own learning experiences. Jacob (2007) goes further to find that most teachers are more effective when working with students of matching race and ethnicity. The challenge this mindset presents is substantial as most educators come from stable, middle-class environments; a stark contrast to the urban dwellings from which many fragile students come. Gomez (1993) noted that, no matter their race, most teachers prefer working in predominantly white schools. This means even those who come from urban backgrounds and become educators still prefer to not teach in those urban settings. So instead of placing these teachers in classrooms where they frequently abandon the profession in short order (Ingersoll \& Smith, 2003), Taylor and Frankenberg (2009) challenge teacher preparation programs to be proactive, providing an alternate path for these teachers within the program or, and perhaps more humane and service-oriented, actively work to 
nurture urban commitment throughout their program. There are efforts being made to link urban schools with the community within which they are situated (Axworthy, DeRiviere, \& Rattray, 2016; Gimbert, Desai, \& Kerka, 2010). In their study of the University of Winnipeg and their efforts to connect Indigenous people and non-Indigenous people in their university town, Axworthy et al.(2016) found that the civic-minded approach of the university to connect its students with the community impacted policy and empowered the Indigenous community by nurturing its strengths. Gimbert et al. (2010) asserts that teachers in training benefit from a cohort structure embedded within the larger learning community. These efforts to connect schools with the community encourage collaborative investment in success.

Noel (2010) suggests work in critical identity theory to explore the power of privileges on urban schools and communities. Through her study of a single urban teacher preparation site, four theoretical frameworks were used to explore the connectedness of the preparation program with the community it served. The four theoretical frameworks were community strengths, theories of presence, trust, and critical identity theory. By collecting data from more common voices, like administrators and teachers, as well as those lesser explored, like parents, support staff, and community members, Noel (2010) was able to examine the layers and levels of community interactions that strengthen the connection between urban communities and the schools that serve them.

Schultz, Jones-Walker, and Chikkatur (2008) shared the results of a two-year ethnographic study investigating what happened when four new urban teachers adopted a listening stance. In a listening stance, the teacher is positioned within the classroom as 
both the informed who has answers to critical components of the classroom functions and also as a learner who is always learning and growing in skills and practices. They reasoned that when teachers are positioned in a listening stance, with both questions and answers, those teachers were able to listen to students and the strengths they bring to learning contexts, listen to the classroom rhythm and respond accordingly to protect the learning climate, listen to the lives of students beyond the school setting, and listen for the potential of home lives to be assets to school learning. They also posited that there is value in new teachers becoming savvy at navigating the listening stance for responsive teaching and mandates from the district that may fully align with the intuitive responses of a listening stance. They concluded that new teachers need negotiating skills and proficiency in explicit teaching in their professional toolbox to encourage retention. They suggest that future and standing urban teachers position themselves as activists who adopt a listening stance where they intentionally seek to actively listen to and learn from the lives of their students (Schultz et al., 2008).

Haberman (2005) suggests that urban teacher preparation programs shift their focus from one fixed on future teachers as their clients to one that focuses on the students these future teachers will ultimately serve as the clients. Many researchers are making attempts to bring clarity to just what it is that urban teachers need to meet with success in urban classrooms. So while the needs of the often most fragile population continue to change and often grow in their level of urgency, the sometimes elusive nature of what works for effectively preparing urban teachers for success remains a viable field of study.

Effective prospective urban educators require both a mindset and skillset beyond those built and nurtured in traditional teacher preparation programs. These tools and 
benevolent dispositions may be nurtured in preparation programs. Donaldson (2009) suggests the need for cultivating in candidates an urban commitment. This commitment implies an awareness of the magnitude of the work for its potential toughness and lasting life impact on the students. There is a level of charity or benevolence without condescending savior mindsets. Donaldson (2009) also advocates for a realistic preview of the work. The level of resilience and grit necessary for effective urban educators should be realized by new urban teachers long before accepting teaching positions in these schools.

Strawn, Fox, and Duck (2008) state, "If we are to prepare teachers to help all students achieve high levels of learning, a new paradigm must emerge in which active collaboration and university partnerships become the learning platform and create a cornerstone for classroom research" (p. 272). These researchers shed light on the impact of a structured, action research frame anchored by immersion experiences for preservice and newly hired teachers. They argue that such collaborative efforts between universities and school districts can lead to experiences for teachers that increase their self-efficacy and improve teacher retention rates in often high-need or hard-to-staff school populations (Strawn et al., 2008). Further research appears warranted to explore collaborative efforts within large urban school districts.

The structures researchers offer to frame collaborative university and school system preservice and induction experiences are varied. Snoeck and Struyf (2012) utilized a framework that included coursework paired with field experiences for secondary preservice teachers who are completely immersed in all aspects of beginning teacher responsibilities at the school level. In their research, teachers were placed as near 
the role of full-time teachers as possible while also provided coaching and mentorship in the field. This experience, or others like it, may align new teachers for successful first year experiences in urban classrooms. These new teachers will hopefully become effective urban educators, those Calbrese, Goodvin, and Niles (2005) describe as culturally responsive, seekers of small successes, encouraging to students, flexible, and caring. They also establish meaningful relationships with students, care about their students' well-being, and see themselves as change agents. The growth mindset is inherent in a classroom where teachers see these attributes as their own.

Several states have responded to the need for specialized preparation for urban populations by developing urban-focused teacher preparation programs. In 2001, Harvard University shifted to an urban schools focus through its Teacher Education Program (TEP), which seeks to better prepare its graduates for urban teaching. However, according to Donaldson (2009), graduates of this program were no more likely to remain in the profession than those educators from prior cohorts without the urban focus. This seems to suggest, at least in this limited study, that teacher preparation for urban schools is simply not enough to prepare and retain highly qualified teachers for urban classrooms. Donaldson (2009) goes on to suggest that universities alone are not able to provide and sustain urban educators in the profession. He recommends that we not look to teacher preparation programs as the lone bearer of responsibility for the retention of teachers. He suggests that more ownership should be taken at the school level "by principals, veteran teachers, teacher leaders and the like - to ensure that these teachers and their novice counterparts get off to a good start in these schools and the profession in general" (p. 
365). He goes on to conclude that urban teacher preparation programs are more likely to work with a university and school personnel collaboration in place (Donaldson, 2009).

\section{Summary of the Literature Findings}

Urban students are struggling and failing at staggering rates. Urban teachers are

fleeing the profession at disturbing rates. Urban school communities are suffering to overwhelming degrees. Urban school districts are posting shameful failure rates for their poor and Black and Brown students. The troubling headlines abound. For this reason, urban education reform policies must continue to reflect honest efforts at changing the narratives of many of urban impoverished and Black and Brown students. Teacher retention is taxing on urban schools. Teachers who depart create conditions that make it difficult for students to receive a quality education. This research study seeks to enter the conversation and the pursuit of solutions by addressing the needs of newly hired urban teachers. The questions of the study are as follows.

1) What are the lived experiences of first-year urban teachers who participate in collaborative, urban-focused induction experiences?

2) What are first-year urban teachers' perceptions of the impact of their lived experiences in the urban-focused induction experience on their self-efficacy during the first semester of teaching?

Through this study, I add to the existing research by exploring the lived experiences of new urban teachers who are supported for greater success through the collaboration of the university, school district, experienced teachers, and the newly hired teachers themselves. I advance that by finding ways to support new urban teachers, we will decrease the urban teacher attrition rates. 


\section{Summary of Chapter}

Developing prospective solutions to the urban teacher turnover challenge rests in the hands of a variety of stakeholders - from policy-makers to parents; from universities to school districts; from school administrators to teacher candidates and the community at large (Gimbert et al., 2010). It will require candid and brutal conversations and actions regarding the needs of our most fragile populations and just how much we are willing to do to address those needs. The challenge is massive but not insurmountable, if meeting the needs of all students is indeed what we truly desire to do (Ladson-Billings, 1999).

In this chapter, I presented literature related to challenges new urban teachers face. Poverty, diversity of student needs, and teacher attrition were explored as great challenges of urban schools. The Community Teacher and Authentically Caring were presented as frameworks for thinking about urban teacher preparation and induction to the profession. Urban teacher competency was introduced as a standard to which urban educators, urban schools, urban universities, and urban communities aspire. All material was presented to provide a context for the work of this study designed to understand the lived experiences of newly hired urban teachers as they are receiving and after they have received some collaborative supports.

Chapter three outlines the methodology of the study, refractive phenomenology (Abawi, 2012). The chapter also outlines the study process and each of the protocols. Data collection and analysis procedures are also presented. 


\section{CHAPTER 3: METHODOLOGY}

\section{Introduction}

In this study, I investigate the lived experiences of newly hired urban educators in the first weeks of their first year of teaching as they engaged in a collaborative induction experience. It is important to explore collaborative models of induction, if newly hired urban teachers are to meet with successful experiences in the ever-changing urban classroom (Gimbert, Desai, \& Kerka, 2010). In an effort to explore the link between teacher preparation theory and urban classroom realities for entering urban educators who are provided a collaborative, urban-focused induction experience, I explored the following research question:

1) What are the lived experiences of first-year urban teachers who participate in collaborative, urban-focused induction experiences?

In this chapter, I present the two conceptual frameworks of the study, study participant profiles, methodology, sources of data, and the data analysis protocol.

\section{Research Approach and Context}

There may be varying ideas about who should be tasked with ensuring the urbanreadiness of newly hired urban teachers or urban teacher candidates. Donaldson (2009) concluded that teacher preparation programs cannot and should not single-handedly shoulder the responsibility of preparing urban educators for the classroom. He asserts that principals, teacher leaders, and experienced teachers must do more at the site level to 
ensure new teachers are supported in getting off to a good start in challenging schools and the teaching profession in general (Donaldson, 2009).

I posit that the responsibility rests with multiple stakeholders. First, the local urban university that is supplying most of the teacher-candidates to that urban district should give significant consideration to the needs of the teachers they are graduating and the students their graduates will be charged with educating. In addition, the urban school district that is entrusted with educating the young to encourage a healthy future for its citizens should be fully committed to supporting the new teachers they hire, and, ultimately, continuously place in their hard-to-staff, high-need schools. Finally, teacher candidates committed to employment within an urban school system must actively engage in building and nurturing their personal poverty, cultural, and community competencies. Many teacher candidates have certain schools in mind when they apply to urban school districts, and rarely do these new teachers seek out a district's most challenging schools (Jacob, 2007). Once teacher candidates decide that they will apply for a teaching position in an urban district, they must seek to prepare themselves for the most challenging urban classrooms because their placement in these classrooms is a likely possibility. The first days or weeks of school, while likely nerve-fraying for any new teachers, should not serve as complete surprises for new teachers, if they are aware of this trend. Knowledge of the challenges would allow them to establish a mindset of active, intentional, and nimble pathways to success. Therefore, the university, school system, and teacher candidates must all approach urban classrooms and students strategically in order to begin addressing high teacher turnover rates if the goal is to truly 
increase the retention rates of teachers prepared to shape the future of the urban community through its children (Gimbert, Desai, \& Kerka, 2010).

\section{Demographics and Context of the Focus School District}

The focus school district includes a student population in its kindergarten through grade twelve program of nearly 100,000 students. The district includes more than 90 elementary schools that average approximately 480 students each. The schools of focus for this study are among the schools within the district that experience lower teacher

retention rates than average. The schools of focus within the study are $67^{\text {th }}, 82^{\text {nd }}$, and $89^{\text {th }}$ in their teacher retention rates among the over 90 elementary schools in the district. Appendix B further outlines the numbers within the district and represented within the study.

The focus school district's traditional new teacher induction experience, The New Teacher Institute, is typically held in the days just prior to the start of the new school year in a traditional professional development format and is required of all newly hired teachers to the district. The structure of the 2017 five-day induction experience is outlined below in Figure 1 (Colley, 2017). Priority Schools are defined as schools performing at the bottom five percent on state assessments. 

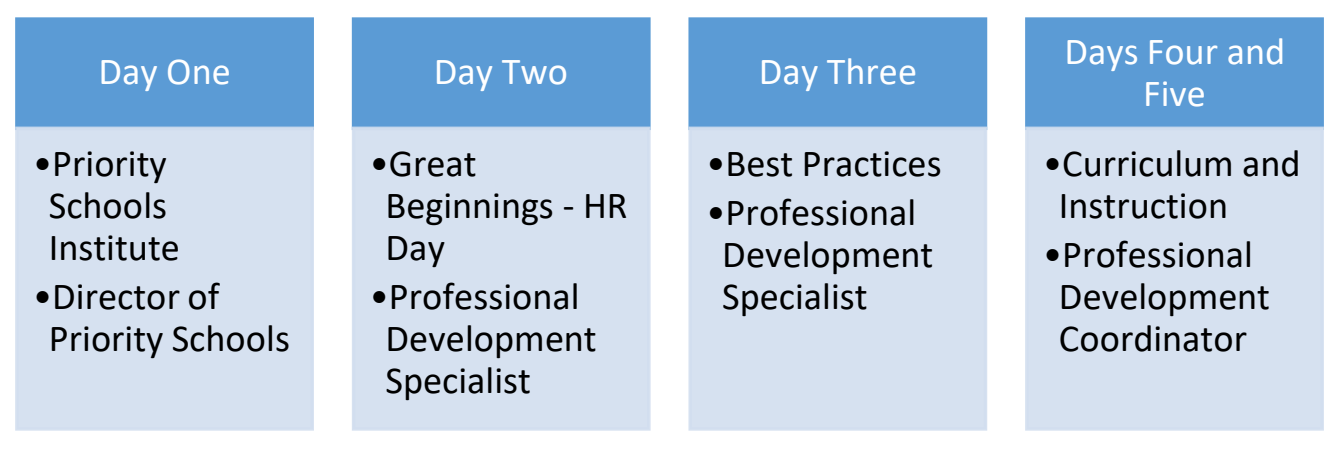

Figure 1: 2017 Five-Day Induction Experience

This research study put into place and explored an additional support for these new teachers once their first year in the classroom was underway through a collaborative new teacher induction model. The study is being conducted in a large urban school district of over 100,000 students that is facing increasing difficulty populating classrooms with teachers who are prepared to meet the needs of students in their classrooms and willing to remain in the education field for the extent of their career (Kentucky Department of Education, 2015). In fact, all three schools represented by teacher participants in this study have teacher retention rates lower than the district average rate of $87.9 \%$. One school's retention rate was $14.2 \%$ lower than this district average. The study provides its six elementary certified participants an additional fall induction experience that is structured to leverage the in-the-field experiences the new urban educators are having by providing urban-focused professional development provided by the local university, the urban district, and by the new urban teachers themselves. The structure of the designed professional experiences are outlined in Appendix $\mathrm{C}$ and include supports in cultural competence, poverty-informed practice, and community-based teaching. Professional experiences were facilitated by university and district stakeholders. 


\section{Refractive Phenomenology}

To further explore the collaboration of multiple stakeholders' investment in new urban teachers that Donaldson (2009) and Simon (2011) suggest, this study utilizes qualitative refractive phenomenological research (Abawi, 2012) to capture the essence of the lived experiences of newly hired urban teachers participating in collaborative induction experiences. This illuminates what happens when all three of these stakeholder groups invest in new urban teacher development.

Refractive phenomenology is a derivative of the phenomenology methodology. Modern phenomenological researcher Max van Manen (2007) poetically describes phenomenology as an artful way of seeing.

Phenomenology is a project of sober reflection on the lived experience of human existence-sober, in the sense that reflecting on experience must be thoughtful, and as much as possible, free from theoretical, prejudicial and suppositional intoxications. But, phenomenology is also a project that is driven by fascination: being swept up in a spell of wonder, a fascination with meaning. The reward phenomenology offers are the moments of seeing-meaning.... [T]he phenomenologist directs the gaze toward the regions where meaning originates, wells up, percolates through the porous membranes of past sedimentations - and then infuses us, permeates us, infects us, touches us, stirs us, exercises a formative affect (van Manen, 2007, p. 12).

Van Manen (1984) explains that phenomenology is also referred to as the study of lifeworld - the world as we experience it immediately. It "offers us the possibility of plausible insight which brings us in more direct contact with the world" (van Manen, 1984, p. 1). 
According to Porter and Cohen (2013), phenomenological research is traditionally approached in one of three ways-descriptive phenomenology, interpretive phenomenology, and hermeneutic phenomenology. Colzizzi and Giorgi are commonly associated with descriptive phenomenology which seeks "to explore and describe the essential structure of an experience in terms of phenomena" (Porter \& Cohen, 2013, p. 182). Interpretive phenomenology aims to examine shared practices and common experiences which are often taken for granted. Hermeneutic phenomenology features a combination of both descriptive and interpretive phenomenology and is commonly associated with the works of Cohen, Kahn, and Steeves and van Manen. The aim of hermeneutic phenomenology is to gain an understanding of how people interpret their lives and come to understand that which they have experienced (Porter \& Cohen, 2013). In their review of phenomenological research, Hein and Austin (2001) describe the hermeneutic structure progressing in these six steps:

Turning with commitment to an abiding concern; investigating the experience as it is lived; reflecting on essential themes; describing the phenomenon through writing and rewriting; maintaining a strong, oriented stance toward the question; and balancing the research context by considering parts and whole. (p. 9)

While Porter and Cohen (2013) describe how hermeneutic phenomenology has been used with regularity in medical studies, this study applies phenomenology to an educational setting with new urban teachers.

The chosen methodology for this research, refractive phenomenology, is a newer methodology based on hermeneutic phenomenology "through the use of interpretive filters taking individual depictions of lived experience and viewing, reflecting, redirecting 
and channeling these through a series of filters and reductive lenses until the 'Essence' of the phenomenon has been brought into view" (Abawi, 2012, p. 141). Refractive phenomenology explores the experience from multiple perspectives and is designed to facilitate new knowledge by bringing lived experiences together, synthesizing them, and then transferring them to wider contexts through its four phases:

(1) depiction - snapshots of lived experiences

(2) reduction to the essence - shifting filters to arrive at the essence

(3) interpretation into living knowledge - synthesis within the context of the study

(4) implications for wider contexts - synthesis related to contexts beyond the study

Depictions are a snapshot of the lived experience. This study will capture participants' depictions or snapshots through four data sources - solicited participant reflective journal entries, focus groups, and a PhotoVoice experience. An example of this four-step process as presented in this study includes the following steps. First, the new teacher creates a depiction or snapshot of a student who steals the classroom supply of crayons. Next, the researcher and/or the participant reduces the snapshot to its essence by the exploration of the event through a series of filters related to the research question. Those filters are both early-determined by the researcher and participants based on the need to explain interpretations of experiences and emerging filters that arise organically as the study unfolds. The early-determined lenses for this study were linked to the urban competency ideas outlined in the work of Murrell (2006) and Oakes, Franke, Quartz, and Rogers (2002). Those urban competencies included an understanding of urban cultures, 
the political economy, the organizational structure of urban schools, the community and social services systems in place to support the community, strategies for building on the social capital found within the community and the ability to establish and grow alliances within the urban community. The early-determined urban competency lenses or filters for this study were the focus of the professional development that participants were provided. They are (1) poverty-informed practices, (2) cultural competence, and (3) communitybased practices. Other lenses that emerged during data collection and initial data analysis — teacher-student connections and teacher self-care—were explored as well.

The sample event captured in the data of this study included a student who takes the entire class set of crayons. This event would be analyzed through the earlydetermined lenses of cultural competence, poverty, and any other relevant lenses to bring to light the essence of that experience. Then, interpretations involving a synthesis of the essence of the experience would be generated by the researchers. So reconsidering the crayons example, the researcher would have a more thorough, multi-dimensional understanding of themes that this event (and other experiences across the study) reveal based on research questions. Finally, the researcher considers implications for other contexts by viewing their findings in other and wider contexts. Continuing the example featured here, this phase may push the researcher to ask: how does this event of a student taking things that do not belong to her (and other experiences captured in the study) relate to other contexts (Abawi, 2012, 2013, 2015)? This methodology is well suited for this research as it allowed for careful examination of the lived experiences of newly hired urban teachers through multiple lenses. The methodology also encourages varied views of urban education that display its intricacies. 


\section{Phases of the Study}

The study was conducted in three phases. Phase one of the study involved the exploration of participants' lived experiences in their first weeks as urban educators. Phase two involved the exploration of the participants' lived experiences as they were provided with induction support and training in three urban competencies - cultural competence, poverty informed practices, and community informed practices. Phase three occurred at the conclusion of the induction experiences, after four weeks of specialized support.

\section{Participants}

A purposeful sampling selection process was used to invite participants into the study (Creswell, 2014). Schools with prospective newly hired teacher participants were identified by central office district administrators. Additionally, a district level administrator provided a list of schools with a higher than average number of newly hired teachers who would benefit from induction supports like those outlined in the research proposal. All of the newly hired elementary level teachers who graduated from a teacher preparation program within the urban area were provided a flyer inviting them to an information session titled Conversation Café where the details of the study were discussed. After hearing the details of the study, those interested were invited to enter the study. Six participants made the choice to engage in the study and all participants completed their commitment to the study. Each participant chose a pseudonym to maintain anonymity.

For the purposes of this study, newly hired teacher participants are defined as certified elementary school teachers from within the focus school district who are teaching their first full year as the certified teacher of record within an urban classroom. 
A brief description of each teacher participant's background and a quote from one of their weekly reflective journal entries during data collection of the study are featured here as an introduction of the group.

Lola is a first-year, first grade teacher hired at the beginning of the 2017-2018 school year.

Although so much went on at home both after and before school [for her], I still went into school with the attitude that I was ready to take on the day and teach the students to the best of my ability. It confirmed my thoughts and beliefs that sometimes even though we are going through so much at home, it is still more important to come into work because so many of our kiddos lack consistency and sometimes, their teacher is their only constant.

Lola is a twenty-something African American teacher who was recruited by the focus district from another state. She and her husband relocated to a suburban area within the urban city for the job opportunity. She is certified in kindergarten through fifth-grade elementary education and is currently teaching in a high-need urban school.

Kay is a first-year, first grade teacher hired at the beginning of the 2017-2018 school year.

This encounter has strengthened my belief in holding students to high expectations and proven to me that if the child knows you genuinely care about them and what's going on-then they will, in turn, care more about themselves. Kay is a twenty-something African American female teacher who was also recruited by the district from another state. She and her significant other relocated to a suburban area within the city for the job opportunity. She is certified as a kindergarten 
through fifth-grade teacher and a graduate from a historically black university. She is currently teaching in the same high-need urban school where Lola teaches.

Gretchen is a first-year, first grade teacher hired at the beginning of the 20172018 school year.

This was a significant event for me because it showed me how vulnerable Joe, and all of my first graders, really are. They are so easily influenced by so many factors in and out of school, and I feel responsible for guiding them away from wrong choices, but I also can only do so much.

Gretchen is a twenty-something White American teacher who holds certification from the local city university in kindergarten through fifth-grade elementary education and kindergarten through grade twelve in special education for learning and behavioral disorders. She lives in a suburban area within the urban city and is currently teaching at one of the district's public Montessori elementary schools.

Junie is a first-year urban teacher hired at the beginning of the 2017-18 school year.

On my way to work two months ago, I was crying out to God to take me out of the job placement I was in. I did not understand how this was where He wanted me to be-in an urban school that I was unfamiliar with and unaware of the deep needs. I was being selfish because God placed me somewhere that made me uncomfortable. Today, I cried on the way to work but for different reasons. I cried in praise to my Father who I know always had me. We moved to this area so that [my husband] could go to seminary. At first, I felt like my only purpose was to be the supporting wife. Now I'm seeing that God is working and growing in me as an 
urban educator that I truly thought I was incapable of being. I'm still working on my "urban readiness" to teach. However, I know I am walking among giants in my field that have opened my eyes to ALL of God's beautiful creation.

Junie is a thirty-something White American wife and mother of two who has relocated to the area as her husband continues his studies. She holds a Master's degree in education with an endorsement in middle school education for science and language arts. She and her family live in a neighboring state in a city commonly considered part of the metropolitan area of the urban school district where she works. She is a second-grade teacher in a high-need urban school.

Chayla is in her first full year as a teacher hired in December of the 2016-2017 school year.

This is the life of an urban educator. We put our best foot forward to get it stomped on, but then use our arms to hug the same child. If I have learned anything throughout this process, it is that there are others like me. There are others who cannot sleep at night because they love their students. We are the ones with cracks covering every part of our body, but continue to move forward so that we may super glue the cracks together on others. We realize that our tired bodies have a purpose. Every day our hearts and minds grow stronger as we help to change not only our students' future but the future of the world. I also know that these are the people that I myself can lean on. These are the wonderful men and women that will understand that I feel as though I may break into pieces. These are the human beings that will listen to all of my unbelievable stories. These are the people who get ME. If I have learned anything... it is that I AM NOT ALONE. 
Chayla is a twenty-something White American teacher who holds certifications as in kindergarten to fifth-grade elementary education and kindergarten through twelfthgrade learning and behavior disorders from the local city university where the study was conducted. She lives near the heart of the urban city and teaches grade five in a high-need public Montessori school.

Anne is in her first full year of teaching after being hired in December of the 2016-2017 school year.

Knowing that this experience could build such a strong community and relationships with my students makes me want to do more. Seeing how many books were out there to make students feel like they are a part of the classroom, like they can relate to other experiences, and they can relate to their teachers makes me excited to dig in and use these books more in my classroom.

Anne is a twenty-something White American teacher who holds certification as a kindergarten through fifth-grade elementary teacher from the university located in the city where the study was conducted. She lives in a diverse suburban area within the urban city and teaches first grade in the same public Montessori school where both Gretchen and Chayla teach.

\section{The Multi-stakeholder Induction Experiences}

Participants in the study engaged in a four-week induction experience after their first three months in the classroom during their first semester of teaching. Participants received professional development over the course of five face-to-face sessions. The sessions include a six-hour opening session, three two-hour sessions, and one six-hour closing session. Appendix C outlines the professional development sessions including the focus for each session and the district and/or university session leader. The sessions are 
aligned with the early-determined refractive phenomenology filters of the study: povertyinformed practices, cultural competence, and community-informed practices.

\section{Session One: Culture, Poverty, and Community}

Over the course of our four weeks together, teacher participants were engaged in professional development experiences and coaching conversations facilitated by university, school district, and peer stakeholders. Day One brought a cultural competence in education presentation from Nyree Clayton-Taylor, Kentucky's 2018 Elementary Teacher of the Year and experienced district educator. She shared her methods of using hip-hop based educational practices to connect with and grow the knowledge of students in her high-need urban school. Teacher participants were captivated by her intentional teacher-researcher moves to ensure students discover a path from their lives to the content of schools. There was also a professional conversation on poverty-informed practices facilitated by Dr. Larhondalyn Mathies, a school-based Goal Clarity Coach within the district and co-founder of a district initiative called Color Brave that seeks to build the capacity of the district to leverage cultural diversity to the benefit of all. Teacher participants were encouraged to challenge their own preconceived notions of students living in poverty. Finally, the session included a professional conversation on community connections with Missy Callaway, district Goal Clarity Coach and Doctoral Candidate at the local university. Missy challenged the new teachers to find a tribe, a support system that will feed their professional spirit, lift them when the challenges arise, and celebrate the joys. This call to act gave a nod to the notion that new teachers are not victims of the challenges of first year teaching. Instead, they were called to actively pursue supports that can fuel their growth. 


\section{Session Two: Cultural Competence through Literature}

Our second session, a two-hour session, featured professional development at my direction. We focused on the use of literature to organically infuse cultural competence in the classroom. I have amassed quite the collection of what I have come to call Close-toHome children's literature after realizing that it is not enough to simply have illustrations in the literature within the classroom that reflect the physical diversity within our classrooms. In fact, it is true that one having the same skin tone, body size, or age of a character within a text does not automatically make that text or character relatable to the reader. It is certainly a step in the right direction after the golden days of Dick and Jane, but I have found that this outward resemblance is simply not enough. Close-to-Home texts are those texts with which I connect because of universal emotions and experiences. For example, I may find greater comfort and connection with a character who has suffered the loss of a family pet no matter the color of that character's skin because I have connected on an emotional level. So, the teacher participants and I explored an extensive library of diverse texts that feature varied life experiences that our urban students may find relatable. Teacher participants were encouraged to comb their classroom library to ensure all students found themselves, their life's journey within the books.

\section{Session Three: Community-Based Resources}

The two-hour third session focused on community-based resources and was facilitated by Social Worker and Adjunct Professor in the School of Social Work at the local university, Harvetta Broadway-Ray. She shared a list of numerous community resources designed for support of the underserved in the city. Teacher participants were surprised by just how many resources there are available and their lack of knowledge 
regarding those resources. She provided contact information for many of the agencies and provided insight into how teachers can utilize those resources to support the whole child. We also spent time watching the 2015 TedTalk featuring Dr. Nadine Burke on adverse childhood experiences. Teacher participants shed tears as some came to realize that they are more like their traumatized students than they had realized. It was a moment of awakening when these new teachers saw their lives as mirrors that actually reflect connections between their lives and the lives of their students, instead of from behind the window of comfort perspective that distanced them from lives like the urban students they were teaching. This shifting of perspective served as an emerging lens in the study, one of teacher-student intersections of life experiences.

\section{Session Four: Poverty-Informed Practices}

Our fourth session, another two-hour session, featured poverty-informed

discussion facilitated by experienced urban educator, Shelli Barber. Shelli shared an article on the topic and encouraged teacher participants to be intentional in their reflective practices to ensure we consider the whole child, not just the many school, district, and state obligations that can sometimes pressure us into behaviors that damage children in the name academic advancement. The reflective discussion allowed teacher participants to openly think with colleagues about challenges related to poverty that are beyond the control of the teacher and the school.

\section{Session Five: Closing Culture, Poverty, and Community}

Professional learning and development for the six-hour session included Dr. Detra Johnson, Professor at the local university, and Dr. Jean Anne Clyde, former Professor in the teacher preparation program at the local university. Each shared her passion for the work of teaching teachers who work in high-need schools and the challenges new 
teachers face at a personal and policy level. Dr. Johnson spoke specifically about urban teacher effectiveness as it relates to both cultural competence and poverty-informed practices. Her passion resembled a sermon that included a call to act in culturally aware and competent ways. Dr. Clyde spoke specifically about connecting with the students, their families, and the urban community we serve through a process she discovered and developed with a team of teacher-researchers that included myself with a strategy called subtext (Clyde, Barber, Hogue \& Wasz, 2006). The subtext strategy was fitting for this study as it challenges those who engage in it to shift to perspectives unlike their own, walk around in that other perspective, and thoughtfully respond to the conditions of the scenario presented. For the purposes of this study, Dr. Clyde engaged us in using scenarios found in urban settings to move beyond our own perspectives to consider perspectives different than, and sometimes at odds with our own. Both professional development experiences fueled reflective and hopeful conversations among participants.

\section{Data Collection}

As the study was conducted, data collection methods included PhotoVoice, participant reflective journal entries, and questions during focus groups which were designed to intentionally pave the way for participants' truths to be made visible to others. Their experiences were more thoroughly studied in the data as the refractive phenomenology methodology examines their truths through multiple lenses.

\section{Focus Groups}

Focus groups are designed to allow the voices of a group to be heard as part of a collaborative conversation. Krueger and Casey (2002) define the key characteristics as 1) a group of carefully selected participants, 2) gathered to interact in a comfortable setting, 3) in a conversation facilitated by a skilled moderator, and 4) later strategically analyzed 
for reporting of the data that resulted. The careful execution of focus groups as the opening and closing of this study allowed for examination of the lived experiences for the study participants.

After confirmation of commitment to the study, participants took part in the first of two focus groups to gather initial insight regarding their self-perceptions of their urban competencies - culture, poverty, and the city's urban community. Appendix D outlines the focus group structure and presents the questions I presented to participants in our opening and closing focus group experiences. The first focus group session was recorded using two audio devices and lasted just under 77 minutes from opening to closing. I facilitated the second focus group at the conclusion of the study after participants engaged in the collaborative induction experience, and it ran a course of just under 88 minutes from opening to closing. This session was also recorded by two audio devices and featured the same discussion questions as those used in the opening focus group to allow for content comparisons during the analysis of data. Recordings of both focus group sessions were transcribed by Rev Transcription service for the purposes of analysis.

\section{Participant Reflection Journals}

Participants generated three solicited reflection journal entries during the study. Each entry was typed by the participant and emailed to me over the course of that week. The entries were generated in response to a two-part general prompt designed to encourage rich descriptions of key lived experiences and reflection on those experiences. Participants were asked to reflect on an event over the course of their lives as new urban educators and to reflect on the significance of that event as it is related to urban education. They were then asked to describe, without emotions, the event from that week. 
Next, they were to analyze the event and their reactions to or thoughts about it, wonderings, and challenges related to it. Finally, they were to discuss whether that event confirmed or discounted their prior perceptions of what it meant to be an urban educator. The document in Appendix E outlines the journal entry structure participants used during the study (Janesick, 2013).

Each of the entries from the participant reflective journals were analyzed according to traditional coding of emotion coding, holistic, and pattern coding (Saldaña, 2016). Entries were also analyzed through the early-determined and emerging filters of refractive phenomenology—cultural competence, poverty-informed practices, community-based teacher, teacher-student connections, and teacher self-care.

\section{PhotoVoice}

The study also included a PhotoVoice (Carlson, Engebretson, \& Chamberlain, 2006; Mohammad \& Sajun, 2014; Wang \& Burris, 1997; Wang et al., 2004; Zenkov \& Harmon, 2009) experience for data collection. A participant-developed PhotoVoice gallery experience is a multimodal qualitative data collection tool that utilizes group content analysis of varied artifacts produced by participants as they chronicle, through the use of images and words, lived experiences during the study. During our third session, participants were presented with the PhotoVoice project and asked to capture images of two significant events in their lives as new urban educators using a readily available device such as a cell phone. They were given a due date for the images to allow enough time for me to have images developed for our PhotoVoice experience that was scheduled for our fourth session together. The images were sent to me digitally. The "artist" of each image created both a title and an artist's statement that gave the image context, like that of a museum piece which includes a placard. The artists' statements were to be brought to 
our fourth session for the event. I printed each image as an $8 \times 10$ at a local photo center. During our fourth session, those images were placed on display for a PhotoVoice gallery along with their artists' statements or placards. The group analyzed the gallery of photos; then, the group arranged them into themes or other categories they noticed based on the collective conversations in a process called codifying (Palibroda, Krieg, Murdock \& Havelock, 2009). PhotoVoice also positioned each newly hired teacher participant as an active participant in the co-construction of their own knowledge and the knowledge of others as they made sense of the lived experiences (Chio \& Fandt, 2007; Strier \& Shechter, 2016).

The images are presented in Appendix G for your observation. These images and the placards generated by the participant-artist were displayed in a gallery where all participants were encouraged to use sticky notes to respond to the gallery. Next, we sorted the images into themes that seemed to exist across the images. When images were sorted and reshuffled, the group established themes. This experience offered fertile ground for conversations about each individual participant's realities and connections within and across those realities.

\section{Data Analysis}

There was a multi-dimensional approach to the analysis of the data collected in this study. The first round of data analysis involved traditional qualitative coding methods found in the works of Miles, Huberman, and Saladaña (2014) and Saladaña (2016). The varied coding structures guided the exploration of themes present within and across the data. First, focus group data was transcribed by Rev and read through for general content. Then, focus group transcripts, participant reflection journal entries, the researcher reflection journal entries, and PhotoVoice data was coded as follows: first 
cycle coding methods that included emotion coding and holistic coding. Emotion coding labels the emotions experienced or recalled by participants or inferred by the researcher within the data. Holistic coding, a macro-level coding method, is designed to allow for the absorption of the overarching content by applying a single label or code to a large unit of data as opposed to line-by-line coding and analysis (Miles et al, 2014; Saladaña, 2016).

The second and third rounds of data analysis examined the data through refractive phenomenology lenses. I read through each piece of data through each of the refractive lenses. The data was explored through both those early-determined filters and filters that emerged over the course of the study. The first refractive read of the data was through the lens of cultural competence. Each event or description was considered through the lens of culture. Next, the data was read through a lens that considers how it was informed or impacted by poverty and poverty-informed practices. This process continued for each of the remaining lenses of community-based teaching, teacher-student connections, and teacher self-care. After each viewing of the events, descriptions, or artifacts through specific refractive phenomenology filters, the data was reduced to allow me to arrive at the themes or essence of the experiences of the study participants. The data was then interpreted to provide understanding of the lived experiences of participants (Abawi, 2012). Figure 2 presents the coding activities of this study. 


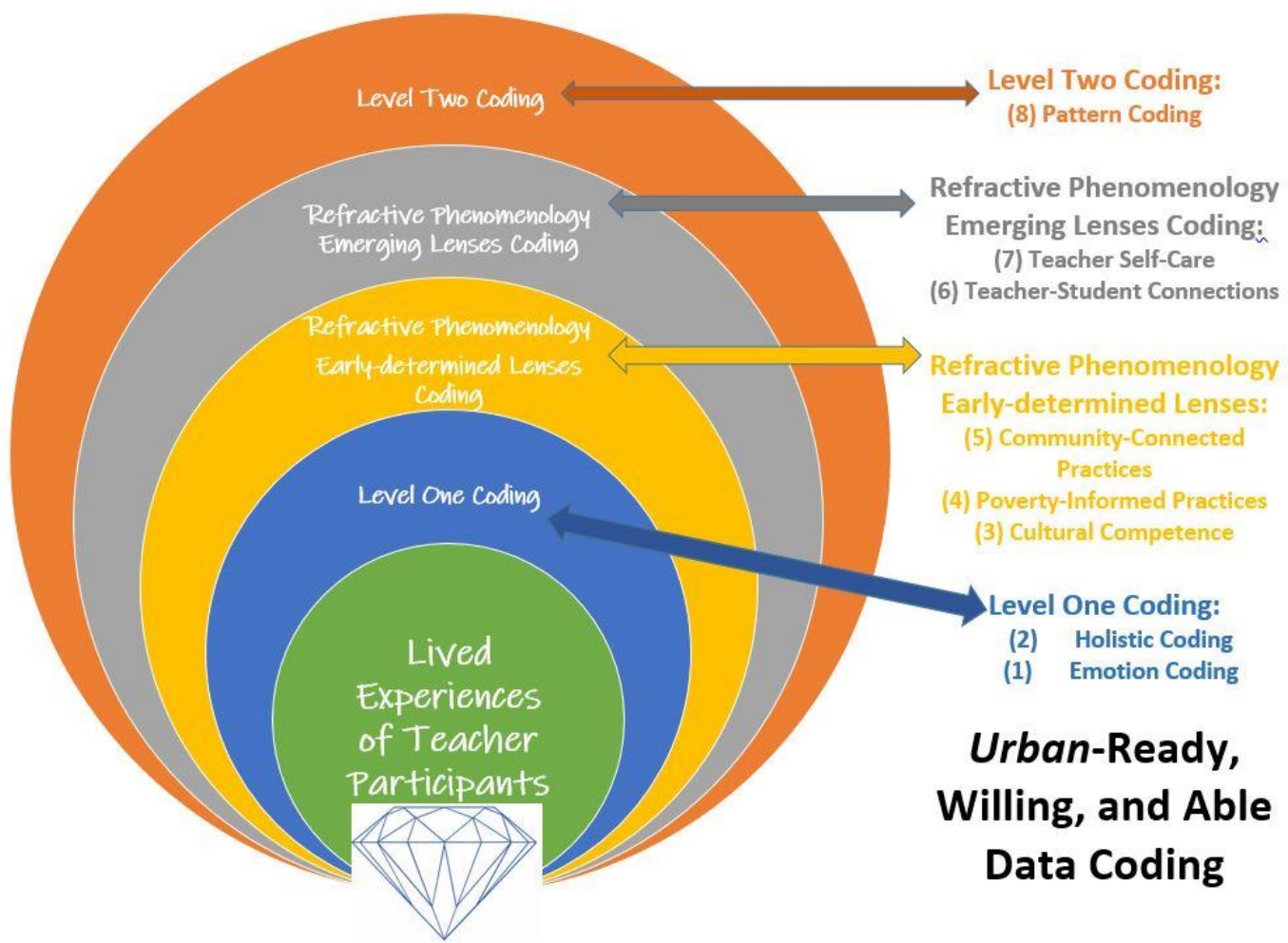

Figure 2: Data Coding

\section{PhotoVoice Data Analysis}

The PhotoVoice participatory analysis used for this study was codifying, the process of sorting the gallery of images into various categories as determined by the artists-participants (Palibroda et al., 2009). This group analysis took place as a collaborative research participant group. Photos were taken of the various ways participants sorted the gallery to allow me to place-hold the varied themes described by participants. The themes that unfolded in the participatory analysis and subsequent reflection contributed to the understanding of the essence of the participants' lived experiences. Emotion, holistic, early-determined filters, emerging filters, and pattern coding were conducted on the images in the gallery as I considered the themes and 
discussion of participants in the PhotoVoice process. The themes that emerged in this researcher analysis were considered in addition to the themes determined by the group.

\section{Pattern Coding}

The full collection of data and themes were grouped into a smaller number of key themes that surfaced over the course of the study. These patterns were considered in the context of the lived experiences of new urban educators in high-need schools. They were also considered in broader contexts beyond those conditions of new urban teachers in high-needs schools (Abawi, 2012; Miles et al,, 2014; Saladaña, 2016).

\section{Validity and Trustworthiness}

Triangulation of the data in refractive phenomenology occurs through the collection of data from multiple sources: multiple participant voices, the collection of data over time, and group participatory analysis. Member checking was built into this study to help ensure that the data reflects the participants' perceptions of their lived experiences and increased the credibility of the study. Each participants' own thoughts and words provided the initial data. Study participants were entrusted to collaboratively define and influence their realities. Fetterman (2017) explored transformative education by looking at empowerment evaluation and Freirean pedagogy. Through this work, people empower themselves through cycles of reflection and action. They perceive and reflect on their realities through dialogue with others and are then empowered to act in ways that impact that reality (Fetterman, 2017). Study participants engage in this type of collaborative work and were then offered the opportunity to review the data of the study and the analysis to ensure their ideas were presented as they intended. This look at the data, both as pieces and as parts of the holistic picture, increased credibility as well. The use of traditional coding methods and the multiple lenses inherent in refractive 
phenomenological research facilitated the examination and reexamination of the data through multiple lenses which increased the validity of the study. Also built into the study was regular informal peer debriefing with a fellow doctoral candidate and teacherresearcher and other urban educators to discuss progress, further process the events, and to consider relevance. The dependability of the study was achieved through the responsive and reflexive nature of the refractive phenomenological study. Without fixed, preconceived notions of what participants might experience, I was able to respond to what the data was revealing as opposed to forcing the data into only preconceived notions established before the start of the study. I utilized the reflective journaling process and the multiple analyzing lenses of refractive phenomenology to also gain confirmability. These many layers of analysis provided structures for the study to maintain the integrity of the data-allowing the stories of these new urban teachers to speak to their realities.

\section{Summary of Methodology}

This study used refractive phenomenology (Abawi, 2012) to explore the lived experiences of six first-year urban teachers in high-need schools as they were provided additional induction support from multiple stakeholders. The results of support from university and school system employees as well as the new teachers themselves as part of professional development experiences was examined in detail. The use of focus groups, weekly reflective journal entries and a PhotoVoice experience provided the data for the study. The use of traditional coding methods as well as those integral in refractive phenomenology allowed for a deep dive into the data to arrive at the essence of the lived experiences of these educators. 


\section{CHAPTER 4: FINDINGS}

\section{A Personal Journey}

It is simply carbon, an essential element of human life - a natural creation born in the mantle of the earth under conditions of both high pressure and extraordinary temperature. Lava eruption forces it near the surface where it is mined from the grip of a hardened rock coat. Diamonds are arguably the most valued precious stone. They dazzle. They sparkle. They shine. Diamonds testify to the journey of the carbon from the mantle layer to the adoration of many in its life as fine jewelry. Yet, there is also the darker side of the diamond story - a blood-thirsty side that is paved by greed, foreign invasion, and human indifference. While not physically traumatic nor as dark, my teacher-researcher journey mirrors the journey of a diamond as it bears witness to both the beautiful and blemished sides of life. My beautifully tempestuous journey not only led me to this study, but it informed many aspects of the research.

\section{Purpose of the Study}

Donaldson (2009) established the argument that preparing new urban teachers for the demands of urban classrooms is the responsibility of multiple stakeholders. He, in fact, found greater success when teacher preparation programs and the hiring school district collaborate to support these new educators (Donaldson, 2009).

In this study, I further explored Donaldson's work calling for a collaborative support system for new urban teachers-bringing together the urban university, urban 
school district, and new urban teachers themselves - as I posited that new teachers must actively and intentionally invest in their own professional growth and development as urban educators. I called on new urban teachers to nurture their own growth because of the common practice among many urban districts that places new teachers in the most challenging urban classrooms. I proposed a notion that lays equal onus of new teacher professional growth at the feet of the new urban teachers. The new teachers' intentional investment is critical to their success and ultimately that of their students. In this study, I explored the lived experiences of these new teachers who were provided collaborative supports that not only included but featured the new teacher as a key contributor to his or her personal success or lack thereof.

To study the collaborative stakeholder investment in new urban teachers that Donaldson (2009) suggests, I utilized qualitative refractive phenomenological research (Abawi, 2012) to explore the essence of lived experiences of newly hired urban teachers participating in collaborative urban university, urban district, and new teacher participant facilitated induction experiences. The diamond proves a worthy metaphor for this refractive phenomenology methodology as it explores a phenomenon through varied lenses or from multiple perspectives. Just as a diamond catches light differently depending on the light source, the angle from which the lighting source originates, and the position of the eye beholding the jewel, refractive phenomenology positions and repositions the researcher to see multiple angles of its "diamond" phenomenon from multiple perspectives. In the study, I also explored the participants' self-perceptions of their readiness for and effectiveness in urban classrooms during their first semester of teaching - yet another way to explore the phenomenon. By increasing or sharpening 
supports for new urban teachers, there may be an increase in their staying power as urban teachers in hard-to-staff, high-need urban schools.

\section{Research Context and Positionality: Researching Self}

Milner (2007) charges education researchers to "engage in processes that reject the exploitation, misinterpretation, and misrepresentation of people and communities of color" (p. 395). I would like to broaden his charge by suggesting that researchers reject exploitation, misinterpretation, and misrepresentations of every population they study. Researchers and those who participate in them should be able to rest easy in knowing that their work has been conducted with sensitivity to and careful consideration of their participants' positions. Calculated misrepresentation of any group is not acceptable, so if researchers adopt a clean hands stance, misrepresentations, exploitation, and misinterpretation can be minimized. This stance is valid for any group being explored in a research study. Yet researchers are human, so their prior influences and experiences, to some extent, impact the way data is interpreted. Keen awareness of the researcher's personal influences should be acknowledged, not ignored. Yet still, intentional efforts should be made to avoid misrepresentations of research subjects. Researcher awareness and intentional efforts to avoid subject exploitation increases the quality of the study.

Specific to hermeneutics that umbrellas the refractive phenomenology stance, Hein and Austin (2001) found in a review of phenomenology literature that hermeneutics "researchers cannot bracket and place aside implicit assumptions and perspectives that are embedded in their own biology and history; rather, they need to acknowledge them and make them explicit" (Hein \& Austin, 2001, p. 9). Additionally, they found that this stance recognizes that full understanding of the phenomenon is not possible, therefore saturation is not possible. Consequently, as researcher, I worked to intentionally lay bare 
my perspectives and prior experiences that I feel are impossible to bracket or exclude from the context of the study.

My selection of the research topic as well as the research structure, practices, approach, and its overall agenda were influenced by several personal cultural experiences. Just as the gaze upon a diamond is influenced by perspective and lighting source, personal experiences continue to shape the educator and researcher I am today. I am an African American female, a child of poverty, an urban dwelling educator, an experienced urban teacher mentor and coach, a single, older mother of minority children who attend the urban school district of focus, and I am personally a product of both the urban school district and urban university where the study is situated. These roles offer perspective and encourage my investment in urban teacher quality. The use of refractive phenomenology methodology positioned and repositioned me in the interpretation of the data to encourage a broad view of the data in ways that honor my positionality while also examining other perspectives.

I grew up as the youngest of six in an impoverished two-parent household where I ultimately became a first generation college graduate. My school career began in this urban district in the infancy of the district's controversial busing student assignment plan. As such, I experienced teachers who were not equipped or lacked enthusiasm to meet the needs of the district's poor, inner city youths. Their training may not have included experiences with the impoverished or culturally diverse students, and they may have felt put upon by the shifts in the diversification of the school's population. I am also the product of two nurturing parents who knew little about ways to ensure school success. Due to these early desegregation experiences, under-informed parents, and perhaps other 
factors, I was retained in grade one - a failure. I was led to teaching by this mildly traumatic experience, and it fuels my desire to invest in urban competencies in teachers throughout the district. So, I was the African American, impoverished, urban student this district serves in high-need schools.

As an educator, new teacher mentor, and instructional coach in a high-need, hardto-staff urban school within the district of focus in the study, I have witnessed firsthand, year after year, the implications of teachers who enter urban schools in our district illprepared to meet the challenges that are seemingly inherent in hard-to-staff schools. Some leave the profession after only a few years. Others struggle to make it through their first year before bowing out of the district and/or profession. Most tragically, I have experienced those newly hired urban teachers who abandon their teaching position, students and the profession in only days or a number of weeks. Beholding this trend and sometimes trying to rescue orphaned classrooms full of students by filling in as classroom teacher is heartbreaking. It always leaves me to wonder what might have happened if there were a strategic plan designed to provide supports to these budding educators that encourages a different outcome — an outcome that includes wins that lead to a career educator who is primed for continuous learning and growing. So, I am truly invested in their success, which often correlates to success for their students and for me as an instructional coach.

As the mother of two young children of color who are currently attending one of the hard-to-staff schools in the district of focus, I am fully committed to doing everything I can to ensure my children's success. That everything includes investing in the success of teachers. Additionally, I am a product of the teacher preparation program at the 
university where this study is situated. My perspective as a graduate of the teacher preparation program at the university provides insight into the pedagogy provided by the university. That everything, therefore, also includes investing in the teacher preparation program of the focus university. I want the university I love, and will be a four-time graduate of at the completion of this degree, to shift its perspective upon the urban school district and to refocus its lens to best prepare new urban teachers for the enormity of the career. While urban universities must prepare their graduates to work in varied environments, current trends indicate that their graduates will be hired in high-need schools. Given the likelihood of this reality for its clientele, I posit that creating a program that prepares them for a high-need school's reality will be more supportive of these new teachers as they gain the traction needed to increase their staying power as career urban educators.

Milner (2007) asserts that researchers engaged in the work of research are simultaneously researching the participants and themselves. I am positioned as a researcher-participant who speaks from both an insider perspective as an urban educator in a high-need school, a parent of African-American children in a high-need, hard-to-staff school, and as a teacher-researcher learning from the experiences of others.

My ideas align with Milner (2007) when he suggests that researcher knowledge of the community or subjects under study is critical to the study, yet the person or persons who conduct the study and the experiences and perspectives they bring to the study must be fully explored. I concur that minimally biased studies can be and are conducted regularly in the field of education when researchers are intentional about ensuring good faith, nonaligned, self-aware understanding of themselves and of those they are studying. 
My researcher position is one of great overlap with those involved in my research. As an African American urban educator whose daily work involves the coaching of new and seasoned urban educators, my study is intertwined with the community. Two of the participants in the study are also African American. This presents further overlap in the community of focus and my position as researcher. I am also intimately familiar with the community of students these educators serve as all participants work in the urban district where I am both a parent and an employee of more than two decades. Additionally, three of the research participants are new urban educators in the hard-to-staff school where I once served as an instructional coach. This organically provides deeper understanding of the community these participants serve and of the experiences the new urban educators face as they enter classrooms in these schools. This up-close connection encourages my researcher perspective to be one of an informed stance regarding the community of students the teachers serve and the teacher participants who were studied. My positioning within the community and district make me cognizant of my perspective as researcherparticipant, but it also increases the relevance of perspective- and lens-shifting inherent in the refractive phenomenology methodology.

Consideration of my researcher responsibilities also requires that I explore potential ethical issues. Specifically, there is an obligation to ensure the work reflects responsible exploration of the research questions and reporting of the findings. As researcher, there is a responsibility to consider the environmental and societal consequences of the study (Brydon-Miller, 2012). As this concept relates to this study, I made an effort to present the information with honesty as well as sensitivity. Challenges existed within the experiences of study participants, yet the study was designed to 
encourage growth and improvement despite those challenges and without lingering emphasis on problems. By adopting this stance, challenges are acknowledged but are not glamorized or overemphasized. Instead, a solution-oriented, growth-mindset stance encourages efforts to find viable solutions.

\section{Study Themes}

Through the shifting of perspectives and lenses, I explored the lived experiences of new teachers in high-need urban schools, and four primary themes tell the story of the journey these teachers experienced. Those themes are compassion, frustration, isolation, and inadequacy. These themes emerged because there was a significant evolution in their frequency or intensity over the course of the study. The shifts in these themes may speak to the impact of the induction experiences provided to the study participants. Looking at these teacher participants' lived experiences over the course of the study through their own voices allows us to better understand their journey, and, more importantly, what might be done to support and nurture new teachers in hopes that they will remain in the profession. I present their journey in three phases-first, as presented during our opening focus group at the start of the study and several weeks into their first year as urban teaches in high-need schools, next, during the study as induction supports were provided from varied stakeholders as evidenced through weekly reflective journal entries and the PhotoVoice experience, and, finally, in the closing focus group as the study concluded. Their journey is presented here, beginning with the opening focus group.

\section{Opening Focus Group Session}

Six teachers and I sit around a large conference table in the meeting room located on the local university campus early that fall Saturday morning where breakfast snacks are served as a token of appreciation for their willingness to engage in a six-week study 
above and beyond their professional commitments in their schools. The energy in the room feels like positive reluctance as they appear willing to cooperate because they showed up early on a frosty Saturday morning, yet not quite sure of much beyond the intent of the study to provide support for new urban teachers in high-need schools. There is also an air of beginning of the year teacher fatigue that I have come to recognize over the years in myself and others. After settling in around breakfast beverages, and warm welcomes, all agree to be both audio and videotaped for the purposes of the study.

The focus group protocol (Appendix D) was designed to unearth participants' personal perceptions of and experiences related to 1) the term urban, 2) the impact of poverty in education, 3) cultural competence, 4) urban communities, and 5) selfassessment of urban-readiness. It was my hope that these categories of questions would allow for a deeper understanding of the lived experiences of the new teachers with some specificity related to these categories. The questions and protocol were designed to invite narrative response and connected conversations among participants as I acted as facilitator.

The group conversation began as each teacher participant introduced herself by sharing their pseudonym, their age in decades, certifications they hold, the neighborhood in town that they call home, and information about whether their K-12 education was public, private, or some other category. (While each participant shared their legal names with one another, they were asked to create name tents containing pseudonyms and to address one another using those pseudonyms during the recording of the focus group conversation.) Next, I asked participants four questions designed to encourage them to share their perceptions of urban education. The questions asked that they define the term 
urban, name the greatest challenges facing urban education, identify the greatest benefits of urban education, and categorize the attributes of competent urban educators.

\section{Opening Focus Group: Compassion}

Teachers who engaged in the study offered a rich collection of narratives which speak to the compassion that brought them to education and keeps them coming back to the work that, at times, might leave those without intimate details of the work cringing under the weight of the demands of these new educators. In spite of the enormity of the work, these teacher participants, time and time again, demonstrate the heart they bring to the lives of students. In the opening focus group Anne's compassion for the work was clear as she reflected, I think you just have to be dedicated because it's not easy. One thing doesn't work, so you have to be willing to keep pushing and willing to keep trying new things; not just to give up and to just walk out on the kids. You have to be dedicated to be there every day, trying something different, doing something different just for those kids. Resilience serves urban educators well, as does the fortitude to approach each day in search of success. These traits draw them to work on a daily basis to do a job that they find significant.

Similarly, Junie's compassion shone as she expressed her concerns for students who go without food or a warm bed for resting. They are not getting food at nighttime, so they're not falling asleep. They're in situations where they're sleeping on the floor with maybe a blanket over them. It's not a safe environment that they're in, so then they can't fall asleep. For those who have never had to go without things they consider basics, it is difficult to imagine students in their care who are living without those things. Junie was not without awareness that these realities exist, but having students in her care who live 
without food and bedding made this reality close and really personal for her and others in the study.

\section{Opening Focus Group: Frustration}

Teacher participants spoke candidly on their perceptions of each question, dovetailing one another with stories of their experiences from within and beyond their classrooms. The weight of their challenges as new urban educators was evident in the ninety-minute discussion. Chayla's explanation of her frustrations seems to encapsulate those felt by other participants as they discussed the greatest challenges facing urban education. I've been thinking about my students and how there are so many different emotional problems that are stemming from home that I have trouble finding the resources. There are students who have divorce. There are students who are in a foster care setting. There are students who have parents coming out of prison. There are students where uncles got shot, aunts got shot, and someone got cancer. There are only a certain number of kids that can be on a caseload for a counselor, and so I feel like a lot of times I'm picking and choosing which ones are the worst problems. The way Chayla expressed the enormity of the work that is not directly related to addressing the instructional needs of the students was indicative of other comments expressed during the conversation. Chayla and others shared insights that illustrate the scope the work these new educators face.

Kay's frustration was coupled with disappointment as she described student behaviors and the lack of support for dealing with them. One of the biggest challenges is some of the behavioral concerns, but further complicating the behavioral concerns is a lack of support for the behaviors - a lack of support in the classroom. There are known extreme behaviors or extreme developmental delays that need more attention than one 
classroom with a teacher can provide. I feel like that's a large deficit—to not have another hand or some type of resource or support in the classroom to help out with those extreme behaviors. Kay's frustration with student behaviors and developmental deficits left her feeling unable to meet the needs of the other students in her class.

Gretchen also captured frustration, but in one example, her frustration was anchored in her lack of context for teaching students whose lives appear distinctly different than her own. She stated, A personal struggle for me with teaching in an urban setting is that I didn't grow up in an urban setting, and to some degree I can't relate to all of my students. The things that they're going through, the struggles that they've had, I certainly didn't experience as a child. And some of it, I have never seen firsthand until I'm in the classroom dealing with it, so that's hard for me. If I'm who they spend all day with, and I cannot relate to them, then it's kind of like, 'How can I make progress with that if we're just not on the same page, and I haven't been prepared to deal with some of the issues that are coming up?' Gretchen felt the urgency to connect with students, yet felt so unlike them that the differences overwhelmed her.

These three examples reflect a sampling of the more than forty narrative incidents within the focus group that capture the frustrations these new teachers were feeling at the start of the study. Those frustrations were rooted in student behavioral and emotional needs, scarce support from administrators and families, and frustrations with feeling disconnected from the realities their students faced. The frustrations left them questioning how they could wade through all of those obstacles to arrive at a place and space where they could teach the content. The weight of the work was indeed a frustration for teacher participants. 


\section{Opening Focus Group: Isolation}

The opening focus group discussion also captured how teacher participants felt isolated in the work. Two examples presented here reflect isolation when dealing with extreme behaviors and isolation from colleagues whose expertise could help these new teachers gain traction. Kay expressed, As a first-year teacher, I'm having behaviors that are dangerous where I'm fearing for my own safety, and I'm finding out that there's little to nothing that can be done. That child is going to stay in your classroom pretty much regardless. I feel like the university should have made me aware of policies and things just so I would know that that child is going to be in my classroom regardless. They kind of make it seem like, 'Oh, if someone is being violent every day or doing this or doing that, there's no way they're going to be in the general education classroom. 'In reality, that's not true at all. Kay's feeling unsafe and unable to ensure the safety of her students was both heartbreaking and familiar to others in the group. Such behaviors were at one time isolated in nature, but are more commonly accepted as normal in high-need urban schools. The need to support these teachers who are facing similar issues is evident.

Lola also felt isolated in her work as new urban teacher. She found herself wishing she had been afforded a more realistic view of what she might ultimately face as a new urban teacher in a high-need school. She expressed, Sometimes I think it would have been a better experience if we had at least been able to observe or help out in a classroom with a teacher who had only been teaching for a year or two. We're not going there necessarily to learn everything from them, but I do think it would be a better experience to just know and be prepared for some of the things that we are going to face and going to be challenged with. Lola, and others who agreed, felt that being able to spend time with teachers struggling to find traction as newer urban teachers as well as 
time with seasoned teachers would have better prepared them for reality. Having experiences with only seasoned educators made working with high-need populations appear too easy. They felt that working with a teacher still stretching to figure it all out would have better prepared them for the most challenging part of their work. They reflected on a longing for a more balance preservice teaching experience that might have better prepared them for the work.

\section{Opening Focus Group: Inadequacy}

The opening focus group discussion also shows the level of teacher participants' feelings of inadequacy as new urban educators. They spoke candidly of feeling like failures in the work. Presented here are three examples. Anne stated, I think that with a changing society, and just the way that students are being raised, and the family lifestyle we didn't really grow up with, and trying to learn that on top of learning to be a new teacher, and not being fully prepared to deal with some of the things that are thrown your way-I think that is a huge struggle. Anne's thoughts captured the sense that she and others were barely treading the waters of new teacher with waning muscle strength and fatigue increasing.

Gretchen also spoke of feelings of failure as a new urban teacher. She stated, There are so many needs that we need to meet and we want to meet. There are just not enough resources to do it. So every choice that we make on what we are going to spend our time, our energies on still feels like kind of a failure because you're not going to be able to get everything done that you want to. Gretchen's comment speaks to feeling that no matter how much they gave, the efforts of these new teachers would never meet their definition of success. These feelings seem reasonable thinking in the minds of those who ultimately walk away from the profession within the first weeks of teaching. In fact, in 
many instances beyond this context, people who feel no possibility of success often opt for cutting their losses sooner than later. Supporting teachers feeling this way may allow for the time and space for development past the most difficult parts.

Junie also expressed feelings of inadequacy as a new urban educator as she reflected on her personal urban-readiness. She shared, I'm one of those people who thinks worst-case scenarios, so I had a worse image in my head for the first week of school than I experienced. So the first week of school, I was pretty pleased. But it's a very transient community, and so since then, I've had more students come in with behavior issues. You have that settling period of the first week of school when everybody is getting to know each other, and it's not as extreme. Now, the behaviors are really starting to pop up. I feel like I'm handling it better myself, but I'm not urban-ready. It's going to be a few years before that happens, and even then, I don't know. Junie's trail of thinking about the movement of behaviors and students in and out of her classroom are reflected in the way she describes her thoughts in the moment. All of the movement, changing, and challenging behaviors led her to conclude that she is not urban-ready and is unsure whether it is even a level or goal possible for her to reach. Junie's thoughts as well as those of Anne and Gretchen provide examples of the self-doubt these teacher participants expressed during the opening focus group. Their participation in the study would helpfully provide supports that would sustain their determination to continue to pursue success for themselves as teachers and ultimately for the learning of their students.

\section{Open Focus Group Summary}

The teachers spoke candidly about the challenges they faced as they sought to navigate the unfamiliar territories of high-need urban classrooms. The feelings of compassion, frustration, isolation, and inadequacy left me with a sense of urgency to 
enhance and nurture successes and support them in navigating the struggles they faced. My power to remove any of the obstacles they faced regarding student behaviors, school or family supports, their preparation program experiences, or any of the challenges is impossible. I did feel, however, called to provide supports that might give them the tools necessary for developing skills that would allow them to learn and grow as educators with the capacity to teach in spite of challenges. In the next section, I discuss these same themes of compassion, frustration, isolation, and inadequacy as participants engaged in the multi-stakeholder experiences provided as part of the induction support.

\section{Induction Experiences: Compassion}

\section{Induction Experiences: Compassion in Weekly Reflective Journal Entry}

The weekly reflective journal entries are filled with narratives that capture the compassion of the teacher participants. In her first entry, Lola wrote about her decisionmaking on a day when an emergency or sick day off would have been warranted due to issues with her car and a chemical spill in her home that made her and her husband physically ill. These conditions did not, however, keep Lola home that day. In an excerpt of her reflection she stated, Although so much went on at home, I still went into school with the attitude that I was ready to take on the day and teach the students to the best of my ability. Sometimes even though we are going through so much at home, it is still more important to come into work because so many of our kiddos lack consistency, and sometimes, their teacher is their only constant. Her dedication to being there for her students while setting aside her personal comfort and needs is inspiring. The selflessness of the teacher participants was heartwarming for me as researcher.

Another example of compassion from the reflective journal entries is one from my researcher journal. It was after the community-focused session which included a Ted- 
Talk from Dr. Nadine Burke-Harris (2015) focusing on childhood trauma and adverse childhood experiences (ACEs). The Ted-Talk conversation includes an invitation to the primarily medical professionals in the audience to reflect on their own experiences to consider personal adverse experiences. Like the participants in the audience, we considered our own adverse childhood experiences. In the discussion after the video, Junie shared, through tears, the realization that she is not so unlike her students; the same students that she felt at stark contrast with based on outward appearances. This teacher was more like her students than she had ever dreamed and, therefore, expressed an understanding of her students that had previously eluded her. Other participants chimed in when we shared our own ACEs and the students in our classes who share those same ACEs. Their lives were more closely aligned with the lives of their students than they had conceived. Participants were able to imagine the possibility of happy endings for their students who, to this point, they had not imagined attaining successful adult lives - the odds were simply too low. The challenges were too great. This single experience seemed to instantly transform the approach or professional positioning of these teachers. Suddenly, thoughts of the school to prison pipeline for their students was at least loosening, if not fully disconnected to reroute to a beautifully possible pipeline of successful adult times. This resonated with me because we are able to care more and better position students for success when we do not see them as so unlike ourselves. Sympathetic views were replaced with empathetic views that could not only have compassion for students, but they could also see potential for the students they were feeling were hopeless for better adult lives. That is powerful positioning for those shaping the future of children. 


\section{Induction Experiences: PhotoVoice Experience Compassion}

The PhotoVoice experience took place in week four using images captured during week three of the induction experiences. Once the gallery was displayed, the group buzzed as they exchanged understanding nods about some images. Then, some perched themselves in front of certain images that seemed to captivate them just as you might see someone taking in a piece of art in a museum that moves them in certain ways. There were moments of silence folded into moments of discussion. Once we pulled the gallery from the display to manipulate them into themes, the need for the protocol questions was replaced with genuine conversation regarding the collection of experiences. The teachers were providing and guiding their own understanding of their realities as new urban teachers.

The PhotoVoice experience demonstrates the teacher participants' level of compassion for the work, their students, and the school. Figure 3 presents three examples of the compassion of teacher participants. In the entry titled, What You Need, I captured an older substitute teacher on the floor where she was giving her all to de-escalate a student who was determined to defy her every request. After the student dropped to the floor in the hallway and refused to move, this invested substitute joined him in an effort to give this student what he needed to get back on course so that learning could continue. Gretchen's entry titled, Roots, eloquently captures her reflections on the need to trust that the work she and other educators do to nurture the growth of students may strengthen the roots, but that time is required for the flower to reach maturity and fruitfulness. The compassion is evident in her hope that, in time, her students will blossom. Finally, Chayla's entry titled, From the Heart, illustrates the compassionate understanding and 
perhaps sympathy she feels for students who are dealing with challenges she, and others, would find unbearable.

The three entries from the PhotoVoice experience that are presented here in Figure 3 serve as examples of the compassion participants felt for students and for the work. Compassion in the experience was also evident in the discussion that took place as part of the sorting and conversation around the themes of the piece. Participants compassionately sympathized and empathized with the challenges of their individual and collective lives as new urban educators in high-need schools. Through their words and artifacts, the compassion felt as though it was resting in their hearts and minds, and this appeared to drive them in the work, not from the work. It seems an essential characteristic of urban educators with longevity in the field.

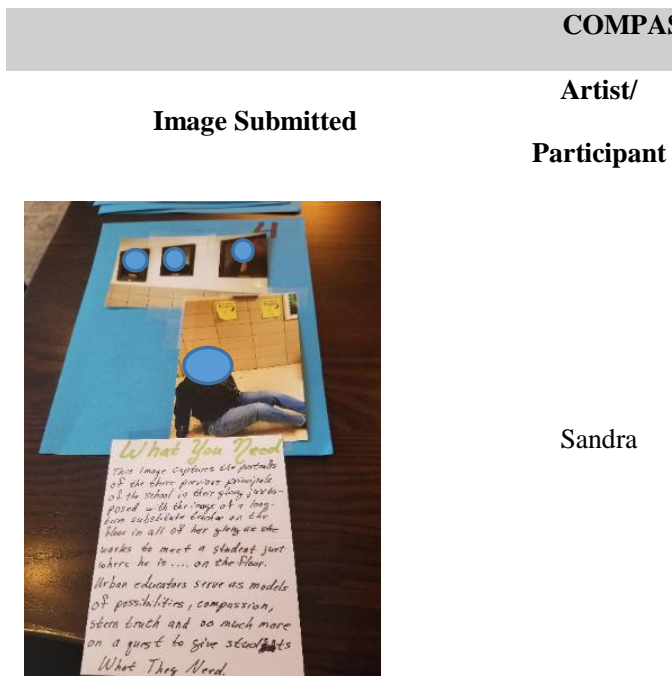

COMPASSION FOR THE WORK

\begin{tabular}{ll} 
Artist/ & \multicolumn{1}{c}{ Artist's Statement } \\
Participant & \\
& What You Need \\
& This image captures the portraits of the three \\
previous principals of the school in their glory, \\
juxtaposed with the image of a long-term substitute \\
teacher on the floor in all of her glory as she works \\
to meet a student just where he is...on the floor. \\
Sandran educators serve a models of possibilities , \\
compassion, stern truth, and so much more on a \\
quest to give students What They Need
\end{tabular}

This image captures the portraits of the three

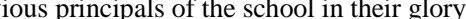
to meet a student just where he is...on the floor. quest to give students What They Need 


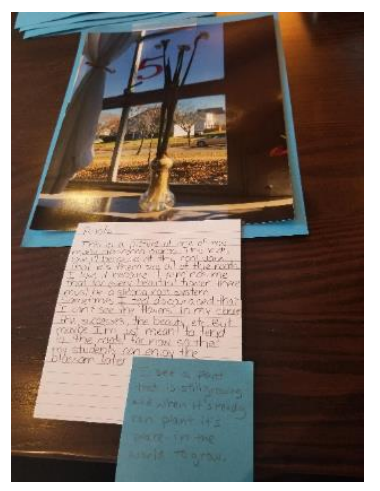

This is a picture of one of my many classroom plants. The kids love it because of the cool vase that lets them see all of the roots. I love it because it reminds me that for every beautiful flower, there must be a strong root system. Sometimes I feel discouraged that I can't see the "flowers" in my class - the successes, the beauty, etc. But maybe I'm just meant to tend to the roots for now so that my students can enjoy the blossom later.

Chayla

From the Heart

This is a student's depiction of a heart. It shows the hardships that she and those she loves deals with. It is how we cope as people. Students in an urban setting deal with more than many could imagine. She draws and uses her artistic ability to express herself. that is still growing, and when it's ready, can plant its place in the world to grow.

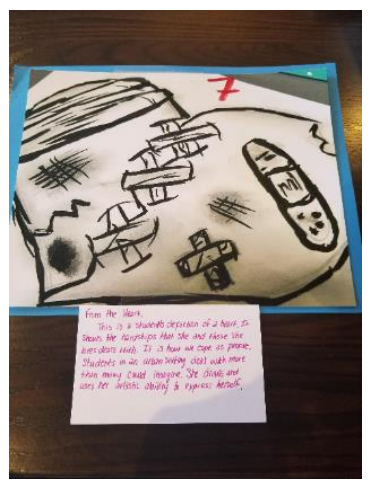

Figure 3: PhotoVoice Experience--Compassion

\section{Induction Experiences: Frustrations}

\section{Induction Experiences: Weekly Reflective Journal Entry Frustrations}

The induction experiences allowed for shared experiences designed to deepen the connections among the new urban teacher participants. Their engagement with the various stakeholders created a support network, but it did not eliminate their frustrations. The reflections from our first week were still largely filled with frustration. Excerpts of three of the weekly reflections will be presented here to provide context and to allow the participants' voices to tell the story of their experiences. First, Anne's reflection illustrates her frustration with an elusive parent who is beyond reach until that parent is forced to reach out for something she feels is urgent, but is completely unrelated to schooling. This frustrates Anne as evident in an excerpt of her reflection presented here.

This past week I was in the middle of teaching and received a phone call from a parent. I was glad that they were calling, but I quickly became frustrated. The parent was 
only calling to ask me to see if their child stole money from them this morning. I was able to briefly talk to the parent on this call and to get their new number, but the only communication with them was something that was not benefiting their child.

I felt so frustrated that the parent's priorities were different than mine. I felt that the parent could call me during my instructional time for something they needed, but they could not answer at any other time. This family contains ten children, and I am aware that the mother works two jobs, so I know that she is not always able to answer the phone or be very involved in each one of her children's lives. It takes a lot of willpower out of me to put myself in this family's situation and not to get frustrated as soon as something happens.

Anne's frustration with the parent who placed great emphasis on reaching out when matters were important to her, but was out of reach at every attempt to reach her regarding the child was evident. While Anne ultimately reflects with some understanding of the challenges this mother must face, she found herself frustrated with this and other parents' priorities.

Anne was not alone in her frustrations. Chayla was also frustrated, but her frustration was rooted in interactions with students. In her reflections on the events that unfolded, Chayla began to realize the degree to which she controls the weather in her classroom — her ability to escalate or de-escalate a situation was becoming clearer to her. In the entry, Chayla describes a thirty minute window of time in one of her days. Her goal was to pick students up from lunch, escort them back to the classroom, and begin teaching a lesson. This seemingly routine and innocent series of events took a negative turn when students who were left behind to assist in cleaning the cafeteria retuned a full 
thirty minutes later and students who were not granted permission to get a drink from the fountain derailed the entire lesson through intentionally disruptive noises and accusations that Chayla did not care about them. Here in an excerpt of her journal entry, we see her frustration with students and herself for not recognizing opportunities to deescalate the situations.

They truly believe that when they are being redirected, it is because someone is out to get them. It is extremely interesting to me, as all of these students hug on me and trust me throughout the day, but once they are in trouble, they distance themselves completely. They immediately react by saying, 'You don't care about me anyway. You want me to fail,' or 'You don't believe in me anyway.'

The students who ran to the water fountain would most likely have reacted much more appropriately if I had confronted them quietly and informed them of why we do not act in such a way. Also, the way that I reacted to the three girls coming into my classroom was completely uncalled for. This shows me that I MUST make sure I am being proactive in all of my behavior management and not reacting with emotion. When I reacted with emotion, the students reacted with their own emotion, and it was counterproductive to the learning for the entire classroom. The only way my students will grow is if I am constantly developing alongside them.

Chayla's reflection on the events in this entry could, on the surface, appear negative. However, the level of reflection and ownership of the turn of events in this approximately thirty minute window of time show her maturity and growth, and it gave me hope for this new teacher working to make meaningful connections with students and to be her best self as she grew with her students. 
Gretchen, perhaps the most reflective of all participants, shared frustrations regarding sensitive events in her first weekly reflective journal entry regarding a student who was beginning to display unhealthy and uncharacteristic behaviors. Upon investigation, she learned that the child's mother had just returned to work after the birth of his first sibling, and that the boy was spending time after school with not-much-older cousins - sometimes without the supervision of an adult. She also learned that one of these older cousins had been caught touching her student inappropriately the prior summer. These conditions, quite naturally, disturbed Gretchen who went on to describe her frustration with the child's fascination with following inappropriate behaviors in school. The older cousin, who is also a student at the school, cheered his young cousin who had just received a behavior referral to the office after hitting a student he had recently been punished for touching inappropriately. Gretchen felt frustration with her inability to rescue her student from conditions that she felt were unhealthy for him. (Please be aware that, while sensitive, the authorities in the school were in contact with all appropriate agencies regarding the child's welfare.) Here I present an excerpt of Gretchen's entry.

Watching Joe's interaction with his cousin really worried me. I fully agree with Joe's mom that his cousin is a bad influence, and I wish that he did not spend so much time with him. It is clear from Joe's behavior that he greatly admires his cousin, and I also feel that his cousin is abusing him —at the very least his power over him as an older kid, but also potentially physical/sexual abuse. After seeing how Joe lit up from seeing his cousin's approval of him sitting in the office, I fear that he will continue to escalate his behavior to try to get back in the office and gain more approval. It is like the first grade 
version of street cred. So many things in Joe's life are stacked against him right now, and I can understand why his behavior took a turn. But I don't have control over those factors, and I cannot fix them for Joe. And if his cousin's affection and approval is what he wants, I am worried that getting in trouble is the way Joe has found to get what he wants.

This was a significant event to me because it showed me how vulnerable Joe and all of my first graders really are. They are so easily influenced by so many factors in and out of school, and I feel responsible for guiding them away from wrong choices, but I also can only do so much. I feel very conflicted about how to approach Joe's situation now. His behaviors have been getting steadily worse, now that he is hitting other students, I cannot put their safety in jeopardy to avoid giving Joe what he wants. I also feel compelled to talk to Joe about good and bad influences, but I don't know how to do that in a way that won't feel like I'm badmouthing his cousin. The situation does make me feel more sure of the fact that in an urban setting, many students need more social/emotional education than elsewhere because they cannot/are not receiving it at home.

While this entry was difficult to read, Gretchen's frustrations with feeling powerless to affect immediate resolution to the student's circumstances reflects the emotion pull new urban educators face. Hired to teach, these educators, many of whom are fresh out of college, are wrestling with far more than the academic wellbeing of their students. Issues such as this one do not have fast and easy solutions. There are many factors to consider, yet beginning urban educators like Gretchen must shoulder these 
issues while also managing to learn and grow as practitioners. That is a heavy load for any educator — seasoned included—but especially for those new to the profession.

Anne's frustration with a parent, Chayla's frustration with students, and Gretchen's frustration with limited power to rescue a student and family she feels are in desperate need are the most compelling of weekly journal entries from week one. Their voices allow us a front row view of their experiences, and, for me, highlight the need to provide supports if we are to hope for careers longevity for these educators.

\section{Induction Experiences: PhotoVoice Experience Frustrations}

The frustrations evident in the PhotoVoice experience include several images that seem to fit in two distinct categories - frustrations with the workload and frustrations with student behaviors. Presented here in figure 4 are the images in those two categories along with the artists' placards and written feedback from the group.
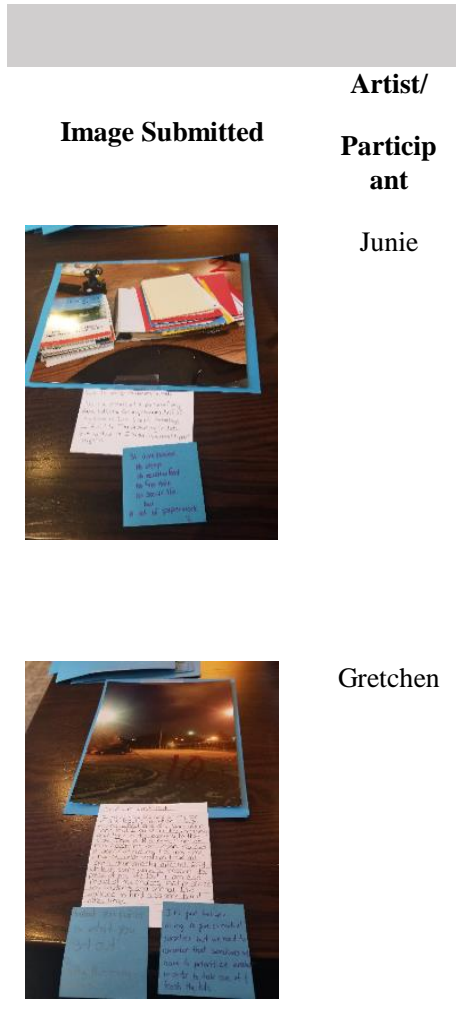

\section{FRUSTRATIONS WITH THE WORKLOAD}

Artist's Statement

Data-Driven or Drowning in Data

This is a picture of a portion of my data collection for my students. Half of my class is Tier II to III. Some days I feel like I'm drowning in data but without it, I know I could not support my kids.

Gretchen

\section{First In, Last Out}

I took this picture of my car in the faculty lot when I was leaving school on day. More often than not, I arrive in the morning and leave in the evening to this view. This is a reflection of how becoming an urban educator is dominating my lifemy time, finances, relationships have all been dramatically affected. And while in some ways I mourn the pieces of my life lost, I am also proud of my choices that prioritize my students and school. I'm
Feedback from PhotoVoice Discussion

So overloaded.

No sleep.

No healthy food

No free time

No social life.

But

A lot of paperwork.

It's great that we're willing to give so much of ourselves, but we need to remember that sometimes we have to prioritize ourselves in order to take care of and teach the kids.

What you put in is what you get out. "Going the extra mile" 
working to find a balance, but it takes

time.

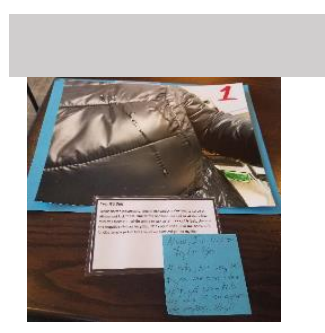

\section{FRUSTRATIONS WITH STUDENT BEHAVIORS}

Lola

\section{Yes, it's Spit}

While on the playground, one of my students decided to curse at other and kick them. Due to the behavior, he had to sit out a few minutes from P.E. While trying to talk to him about his behavior and the negative choices, he yelled, " $f^{* *} k$ you" and spit at me. Most of it landed on my jacket, but a small amount did get on my face.

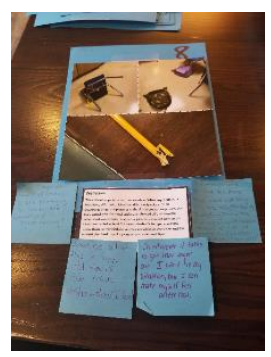

Lola

\section{Destruction}

This collage has pictures from various days. When angry, upset, or frustrated, different children found their only release to be destroying things and property in the classroom. In one picture, you see a pencil with the metal and eraser chewed off and then the pencil itself was chewed. In another picture, a student went to our closet and pulled at least six different students' backpacks out and threw them. In the third picture, you see a kicked over chair and the student also tried to pick up one of our tables and flip it.
Now, I've Got to Fight You!

Not really, but very little angers me more than spit, yet some kids feel this is an appropriate response. Why??
Imagine what this 6 year old feels like inside \#showhowIfeel

Do whatever it takes to get that anger out. I can't fix my situation, but I can make myself feel better.

Even though her heart is broken, I see her trying to care for the wounds but is there healing?

This reminds me of the emotions that come out daily. Why are they throwing everything? Why the fascination with broken pencils? Is it all so someone finally notices them, And is this their only voice?

But you have to teach them that you work to supply bins. Students are able to get what they need if they are missing supplies to complete an assignment successfully. While I was out of the classroom, one of the students took the entire bin of crayons by putting them in his backpack and taking them home. The rest of the class was very upset that they no longer had crayons to use. The student's reasoning for stealing the crayons was that he did not have any at home and wanted them. Some students in an urban society have learned that in order to get something you want or need, you can take it. earn what you want.

Crayons were not meant for drawing. They are meant for throwing.

\#FindingBrokenCrayonsEverywhere

$\underline{\text { Some }}$ and sometimes it's not economic poverty-related

Figure 4: PhotoVoice Experience--Frustrations

Junie and Gretchen created pieces for the PhotoVoice experience that place hold

the frustrations of the workload involved in their experiences as new teachers. Junie's

entry in the PhotoVoice experience, both image and artist's statement, captures the

realities of paperwork in today's urban classroom. She and others in the group discussed 
their surprise regarding the amount of paperwork, beyond merely planning for instruction, involved in teaching. Gretchen's entry speaks to her efforts to find a worklife balance that, to date, had eluded her. These entries and the subsequent conversation around them demonstrate the frustrations these new educators face in their efforts to not only do it all, but to also do it all well. The sense of barely treading water presents itself in their experiences once more.

There were also frustrations in the PhotoVoice experience related to student behaviors. Both of Lola's entries and one from Anne provide context for these behaviorrelated frustrations. Lola's first image of the spit on her coat at the hands of a student epitomized her frustration with extreme student behaviors. She also provided a collage entry that captured destruction of the classroom at the hands of an angry student. Anne's entry spoke to her frustration with a student who chose to take the community supply of crayons, leaving the class without any. Each of these entries provide examples of the types of incidents that frustrate teachers, especially new urban educators who have not yet developed de-escalation skills.

\section{Summary of Frustration during Induction Experiences}

The participant weekly reflective journal entries and entries shared in the PhotoVoice experience provide a glimpse of the frustrations teacher participants felt during the study. The way they share their struggles provides other stakeholders with a deeper understanding of what makes being a new urban teacher in a high-need school difficult. With intimate knowledge of their struggles, stakeholders are better positioned to support them through the most difficult parts. 


\section{Induction Experiences: Isolation}

\section{Induction Experiences: Weekly Reflective Journal Entry Isolation}

There were two weekly reflective journal entries that provide insight into the turn of emotions regarding the seclusion teacher participants began to experience over the course of the study. There appears a metaphorical squaring of the weary and worn shoulders and linking of the arms in the words of these entries that capture the sense of connectedness that began to grow. Lola's second entry demonstrates her growing connection with her students as part of a classroom community that she began to nurture through literature. She seemed to bask in the connections and delight her students felt with the text she read. She also demonstrated pride in her growing teaching craft facilitated through her connection to this new teacher experience. An excerpt of her entry is presented here.

The children loved the story and so did I. This is so significant because children need to see themselves in the books we read, and this gave them an opportunity to do so. It motivated me to choose literature that my students could better relate to. I am definitely working on a way to weave close to home literature within my lessons, not only for the students, but for myself.

Lola's entry captures the excitement she felt with the newfound knowledge of the power of literature to connect students to one another, teachers to students, and teachers to other teachers. Knowledge is powerful. Lola's entry captures just how she is beginning to see ways to draw her classroom community closer together, and it captures her joy of feeling empowered as a new educator who has connected with new tools through connections with other educators. 
Chayla's third and final weekly reflective entry speaks to her turn from feeling isolated and inept toward feeling part of a community of educators empowered to change the world. She and others in the study began to feel a sense of connection as a group of educators committed to giving their all for students who need them. The sense of tribe began to emerge more clearly in the group. Chayla's entry captured the narrative of a student who frequently took things that did not belong to him. On the day she describes, he took her cell phone and wallet case containing credit cards, identification, and other important personal items. The phone was returned, but the wallet and all of its contents were gone. Her entry mentions her disappointment, but largely expressed her worries for a student who was coming from a place in his life where he felt this choice was wise. Her concern for his path in life overshadowed her disappointment. Presented here are her reflective thoughts on the realization that the work is tough, but she is not alone, and she is willing to remain dedicated to her calling to be her best for students.

Linda Poindexter once explained, 'One small crack does not mean that you are broken. It means that you were put to the test and you didn't fall apart.' This is such a short phrase, yet it represents so much of what is urban education. It not only represents our students, but it represents us as teachers. [Teachers] put away their emotions in order to become the best advocate, the best mentor, the best educator possible. Consequently, these same advocates, mentors, and educators become a victim of the illness, Post Traumatic Stress Disorder.

Ifelt the need to share these understandings, as I have recently begun to comprehend the magnitude of our situation as urban educators. Initially, I had the thought that I was alone in my struggles - that my students acted as they did in the 
hallway and in the classroom, because my management as a teacher was off. Maybe I was too close to my students? Or maybe I didn't connect with them as I thought I had? The stress was overwhelming, but I did not want to share this with anyone, as it was almost like as admission of defeat. It felt as if I expressed my worries to another teacher or supervisor, that I was lessor, or that I was not competent. Not until I was placed in a group of new urban educators, did I learn that I am not alone. Through learning new techniques and sharing with others, I learned that my struggles are not uncommon. I learned that it is ok to have these issues, and that everyone has them. I learned that brainstorming and sharing with other teachers can actually be the most therapeutic experience possible.

This is the life of an urban educator. We put our best foot forward to get it stomped on, but then use our arms to hug that same child. If I have learned anything throughout this process, it is that there are others like me. There are others who cannot sleep at night because they love their students. We are the ones with cracks covering every part of our body, but continue to move forward so that we may super glue the cracks together on others. We realize that our tired bodies have purpose. Every day our hearts and minds grow stronger as we help to change not only our students' future, but the future of the world. I also know that these are the people who I, myself, can lean on. These are the wonderful men and women who will understand that I feel as though I may break into pieces. These are the human beings who will listen to all of my unbelievable stories. These are the people who get ME. If I have learned anything... it is that I AM NOT ALONE. 


\section{Induction Experiences: PhotoVoice Experience Isolation}

The PhotoVoice experience discussion is the best evidence of the group's growing turn from isolation toward connected community. The entire experience could be metaphorically described as the bundle of fragile sticks in the Kenyan proverb that says: sticks in a bundle cannot be broken. Those sticks, those new urban educators, collectively represented strength. These new urban educators were empowered in spite of the struggles because they were coming together to share trials, knowledge, and experiences as a bundle. For example, as the group sorted the images from the gallery, at one point they placed the two images together, explaining that they were connected by

theme. When I asked them to explain the grouping, they described how the of mounds of paperwork often leave them to be the first cars in the school parking lots in the morning and the last cars to leave at night. They all agreed that this was a familiar, undesired scenario. While there was evident frustration with this far too common reality, the comradery they shared as they discussed it caused me to reflect on the comfort they felt in understanding that this frequent occurrence was not a reflection of their weakness as new educators, but dedication to the work.

\section{Summary of Isolation during Induction Experiences}

The teacher participants seemed to shift in their feelings of isolation as they experienced the induction experiences of the study. The opening focus group featured Lola and Kay describing how they felt alone in their struggles with student behaviors and isolated as they wrestled with the realities of urban teaching that are in contrast to their teacher preparation experiences. The sense of loneliness as educators shone through the words of many participants. These entries that were shared during the induction experience are not without frustration over numerous challenges, yet there is a turn 
toward kinship as part of a tribe of educators committed to learning and growing together by sharing struggles and gifts with one another.

\section{Induction Experiences: Inadequacy}

\section{Induction Experiences: Weekly Reflective Journal Entry Inadequacy}

While the induction experiences seemed to be having an impact on the new urban teachers' sense of isolation over the course of the study, there were still multiple incidences that bring to light their feelings of inadequacy because of the enormity of the work. One such example is found in Junie's third weekly reflective journal entry. In it, Junie describes how her newfound knowledge of close to home texts led her to a local used bookstore where she combed the shelves for books that would resonate with students. While there, she encountered a woman who pegged her as an educator, but the woman then asked what books she would buy for her own children. Junie was struck by the fact that her own children, who love to read, never entered her mind on this shopping trip. This helped Junie to reflect on our experience on teacher self-care and the importance of not neglecting the other important parts of her life.

Junie's entry provided us with the reflective thoughts of an educator who feels she must give her all professionally, but she has somehow lost focus on her own children. Junie and other new urban educators struggle to find a healthy balance of being their best as a new educator while maintaining their presence and effectiveness in their personal lives. This juggling can certainly be a source of stress for new educators that is hard to manage.

Gretchen provides the second example of the feelings of inadequacy new urban educators in high-need schools may face. Again, Gretchen is thoughtful when faced with a difficult scenario that unfolded in her classroom. The sensitive content is presented here 
in Gretchen's own thoughts and words and is the only weekly journal entry presented in its entirety to reflect the gravity of the experience.

My reflection this week is about an incident that occurred last Thursday during school. The class was in the middle of afternoon work cycle, with most students working independently at their seats while I had a small group with me at my table. My students have the freedom to be up and around the room as needed so they can get the work and supplies they need. Several students were getting up to come tell me that a male student in my class, we will call him Jim, said he had a cigarette he was going to smoke at wellness. The first two students who told me this are students who frequently lie and make things up about others, so I told them to get back to work and did not think much of what they were saying. However, after a very trustworthy student came and told me the same thing, I called Jim over to my table.

I asked Jim if he had brought anything to school that he shouldn't have, and he said no. As he denied it, I saw that he was using his hand to cover his pocket. I asked him to show me what was in his pockets, and he quickly displayed all of them except the one his hand was protecting. I told him I needed to see what was in that pocket, and he said nothing, so I reached out and thumped the pocket with my fingernail. I could immediately feel there was a lighter inside, and I told him he had to give it to me. He did, and I asked him if he knew what a lighter does. He said that it was for smoking cigarettes. I told him that was true, and that lighters make fire. I explained that making a fire at school would be very dangerous, so I was going to keep the lighter and he should not touch one at home again. He agreed, and I sent him back to work, setting the lighter aside on the shelf behind my desk. 
About ten minutes later, I had two students in quick succession come up to me again saying that Jim had a cigarette as well. I called him back over and told him I needed the cigarette, too. He swore he didn't have it, but I pointed out that he wouldn't have brought the lighter to school if he didn't have something to use it on. He then quickly told me he had given the cigarette to Joe (from my last reflection). I called Joe over as well and asked him if he had the cigarette, and he said yes and quickly handed it over to me. It was a small, brown, and squished nub of what had once been a hand rolled cigarette. I took it from Joe and told him to wash his hands.

When I had sent both boys back to work and released the students from my small group, I began examining the nub. I sniffed it and used my fingernail to pull open the end a bit, and upon seeing the contents were green realized that it was not tobacco but marijuana. I called the office and asked for assistance because I had confiscated inappropriate materials. A few minutes later the principal, vice principal, and behavior specialist were all in my room. I turned over the lighter and nub to the principal and explained how I had gotten it and who had brought it in. She took the items, Jim, and his backpack to the office. About an hour later, the counselor came in and watched my class, and I was summoned to the principal's office. There was a police officer there who had tested the nub and confirmed that it was marijuana. I gave a statement to him for his records, and the principal explained that because there was so little substance in the nub and Jim is only six years old, there wouldn't be a police report, but we would need to file a CPS report. She also told me that the reason that the boys' bathroom had been shut down earlier that day was because someone had been smoking in there before school. 
This event broke my heart. When the situation began and I thought we were talking about an actual cigarette, I didn't think it was a big deal at all-Jim might have slipped a cigarette out of his mom's pack that morning and brought it to school to look cool. Jim lives in a single parent household with his mom and two brothers. His father passed away last summer. He and his brothers are all such sweet and caring boys, and I never had any trouble out of Jim at all. He is a model student when it comes to behavior. After it came out that he had brought drugs to school instead, I was overwhelmed with a lot of feelings. I'm wondering where he got it, who he is around who smokes it, if that person smokes in front of him and calls them cigarettes. And in a lot of ways, I feel angry at this person--angry that they are exposing Jim to drugs, that they are careless enough to leave them laying around where the kids can take them, and that this person potentially smoked on school property.

But more than the anger, I feel worried for Jim, his brothers, and his mom. I have only spoken to Jim's mom once, at the open house the week before school started. Since then, I have tried to contact her several times, but she doesn't answer her phone or return calls, and she never responds to notes in his folder. I haven't been bothered by that because Jim is such a good student that I never have anything urgent to discuss with her anyway, and I know she must be busy and stressed after becoming the sole caregiver for her family. I don't know if the drugs belong to her, but if they do, I find it pretty easy to empathize with her even if I don't approve. She is a recent widow, raising three very good boys on her own. Maybe that's how she copes with what's going on in her life, and if it is, then I don't feel it's my place to judge her for that. And maybe it isn't hers at all. Maybe it belongs to another family member, a friend, or someone who drives the boys to 
school. I really have no idea who all Jim interacts with outside of school. But I know that no matter whose it is, there will now be a CPS investigation. I have no experience with CPS. This was my first report filed. I'm scared that Jim is going to be removed from his home, his mother, possibly his brothers because he brought something to school he shouldn't have, and I turned it in. I feel responsible for disrupting his life, and for betraying his trust in a way. He had to be interviewed by the police officer, he would, no doubt, have to talk about what happened when he got home, and social services is going to become present in his life. And in his six year old eyes, that might have all happened because his teacher got him in trouble.

Now I don't know how to act around Jim or how to address what happened with his mom if I ever hear from her. When he came to school on Friday morning, he seemed like his normal self, so I acted normal too, and I guess everything is fine. But it's still weighing heavily on me. I also wonder if his mom will contact me and acknowledge the situation. The principal called home so I wouldn't have to, but I don't know how that conversation went. I keep trying to guess how she and Jim's brothers feel about the situation and me, and I just can't guess. I've also been thinking a lot about how when we talked about filing CPS reports in college, I always thought that it would be a pretty easy thing to do-like it would feel like a good deed, because it would only be in situations when a child was being neglected or abused, and I was helping them. Now I see that in reality, nothing about the situation is easy. Even though I understand that I had to turn in the items, and I had to file a report for Jim's safety and well-being, a part of me also wishes that I didn't do it because I feel so guilty for Jim and his family and the consequences that might come. I think this is an example of how my expectations for 
teaching in an urban school were both accurate and so off base. I knew I would have to deal with CPS reports, and neglect, and abuse, and illegal actions. I didn't know that I would be so conflicted over those things and where the line really falls between being not-the-best parent and being a danger to your child.

Gretchen's emotional and compassion-fatigued recount of the events that unfolded in real time, the subsequent legal components, and the reflections presented here serve as a prime example of the connection she and others educators experience. If not balanced with sufficient self-care, these experiences can fray the emotional health of educators and fuel feelings of inadequacy. This and other experiences over the course of the study reminded me that teaching in high-need urban schools is not for the faint of heart. This is true even for new urban educators who must quickly develop the ability to find ways to be effective even in times such as these when they are conflicted by the need to follow protocols and law with compassion and professionalism—all while avoiding feelings of helplessness and inadequacy.

\section{Summary of Inadequacy during Induction Experiences}

The words of Junie and Gretchen provide us a deeper understanding of the conflicting feelings these new urban educators faced over the course of the study. While they appeared to be more united in the work, they were also still dealing with feelings of inadequacy to effectively do all they were being called upon to do. This signals to me that support for them is crucial to the hope that they will remain in the profession long enough to develop maturity in their craft.

\section{Closing Induction and Focus Group Session}

On the final day of the study, teacher participants returned to the location of our opening focus group. The energy was different as the interactions mirrored those of both 
colleagues and friends. We had cried together, laughed together, learned together, and grown together as a tribe of first year urban teachers working in high-need schools and those who support them. The focus group questions mirrored those of the opening focus group in an effort to explore a before and after comparison of the themes that might emerge. Just as I have done with the opening focus group and induction experiences, I present here, a look at participants' lived experiences as they relate to the themes of compassion, frustration, isolation, and inadequacy. Through these themes, we will be able to compare participant perceptions prior to the induction supports of the study and after the induction experiences.

\section{Closing Focus Group: Compassion}

Coding of the closing focus group demonstrated greater compassion for students' lives. There was understanding of the challenges their young students and their families faced. In their excerpts Anne, Lola, and Kay show their authentic care and compassion for their students. Anne describes compassion for a student who copes with extreme poverty by deflecting attention onto others. She stated, I know that my student in poverty doesn't want others to joke on him because he smells or because his shoes are broken or anything like that. So he jokes on others first. We just have to be aware of that and know why they are doing it. Even though the six year-olds don't understand why he's joking on them, he's doing it because he doesn't want it to come back on him. As a result, Anne was working with the student on building relationships instead of simply punishing him for bullying behavior. Restorative practices are more compassionate, by design, than punishments. Anne's thoughts capture the compassion in her approach to the student's behavior. 
Lola spoke of the importance of slowing long enough to hear from students. The hustle and bustle of the school day, the tight schedule, the paperwork, the monitored instructional minutes, the disruptive behaviors, and the herding of twenty-four little bodies through the classroom and school may cause us to lose sight of the fact that sometimes students just need to be heard. In her example, Lola describes a student who queried whether she had watched the nightly news the previous evening. She admits to a flipped quick "No, I didn't watch the news," in her response, and proceeded to set the course for the day's activities. What happened next is presented here along with Lola's reflection on the need to show compassion for students dealing with things far heavier and arguably more important than our curriculum. She stated, She waited for me to respond more, and I just didn't. And she said, 'If you had watched the news, you might have seen that somebody got shot, and you would know my cousin got shot.' I was thinking, what do you even say to that? So I stopped what I was doing, and we sat down and had a conversation about it. Sometimes the questions kids ask you may seem insignificant, but it is of the utmost importance to them. So you really have to take a step back and pay attention to what they're asking because you may think it's completely unrelated, but it really means everything to them. Lola's compassion for young people coping with traumatic experiences shows the importance of slowing down to care for the whole child, not just their academic needs. Sometimes their hearts need nurturing as well.

The final example from the closing focus group discussion was shared by Kay who described the compassion she feels for her students. The example she shared was about a student whose behavior that week on the surface was defiant and disrespectful. Kay suspected that there was something amiss and attempted to connect with the student 
by asking about whether something was bothering her. The student refused the conversation and continued the escalating behavior. Kay later discovered the trauma that spurred the child's actions. She described, Over the weekend, she was with her dad, and he was arrested. So the police put her in the back of the car with him and took her to the station where she waited for hours. They had to find out who her mom was and wait for the mom to get there. So she spent several hours in the police car and at the police station with her father, and she's six!

I would be upset too. And so when I found out, I talked to her about it. I would have never known if the other teacher hadn't told me. Kids are not always going to come and tell you why they're acting out. You never know what they've been through over the weekend. It really could be anything. So I think it shows in some of the behaviors. The compassion that came with Kay's understanding of the events of the weekend was also rooted in personal connection as her father had also spent time incarcerated in her childhood. The ability to connect with the lives of students, both the joyous and painful, brings students and teachers closer in ways that nurture a community where learning of content occurs in spite of and sometimes through the connections made in the classroom community.

\section{Summary of Compassion in the Closing Focus Group}

Urban teachers in high-need schools must ensure their efforts to educate their

students are not marred by indifference to the personal lives of students. Students are not all the same. People are all different, but all of us are largely shaped by our experiences and the things we have been exposed to. Many of us are jarred by the trials some of our students face, yet it is through compassion that these new urban educators are guided to seeing the whole child, not just the data being monitored by those on the periphery. The 
compassion, of course, must not become an excuse for lower expectation. Instead, compassion coupled with direction allows for caring for the whole child.

\section{Closing Focus Group: Frustration}

I wish there was a way to remove the frustrations new urban teachers in high-need schools face, but the reality is there no such magic. However, I believe there are ways to lessen those frustrations. There was a noted difference in the coded narratives of frustration in the opening and closing focus group. In fact, the number decreased from over forty incidences in the opening session to fifteen in the closing session. Presented here in the words of Chayla, Junie, and the group in a rapid-fire exchange, are examples of the continuing frustrations they were facing.

Chayla expressed frustration with the cycle of poverty and the connection of poverty to gangs and gang violence. She expressed, I have students in my classroom who have recently had family members die because of gang violence. So I think that gangs are also a problem. They start because people are looking for money, because they are looking for easier money. Many families are really struggling because they don't have the income from the lost gang member. Chayla's frustration with the cycle of poverty, and more specifically, its impact on her students' lives is evident. While wishing there was an easy solution, the magnitude of the challenge is one that Chayla understands to be one of the factors beyond her immediate control.

Junie expressed frustration regarding gang violence later in the discussion. She was frustrated by student fascinations with violence and guns and connected this fascination with negative outside influences in the urban community. She discussed her students' fascination with using classroom tools and manipulatives to gleefully build guns. Junie's frustration regarding behaviors she assesses to be gang-related is evident in 
her statements. The thought of these influences on her students are clearly disturbing to her.

The most heated expressions of frustration during the closing focus group were found in the discussion regarding how new urban teachers should be prepared for the demands of urban classrooms. The conversation included pointed feedback for teacher preparation programs. They advocated for teacher preparation programs that expose students to a more realistic preview of the work they will ultimately face. Lola stated, Put them in difficult classrooms while they are still being taught. Do not always put them in a room with somebody who has been teaching for 35 years with four Master's degrees and a Doctorate. That is not what they will see, deal with, and experience every day. Sometimes they should put them in a room with somebody who has been teaching for six months and has a really difficult class. If they can handle that, they would have a few strategies to help them deal with it later. I feel like I went in so unprepared and with such a lack of understanding of what I would actually be experiencing in an urban classroom. Chayla continued, It's almost unfair to put them in classrooms where the teacher knows exactly what to do all the time, and the kids are on point all the time. Then you get there, and it's like a bomb goes off. Anne reflected, I'd rather fail when I have another teacher right there helping me to solve it instead of waiting until I'm on my own. Instead of just teaching one lesson and then sitting back down or just observing, make it realistic. Finally, Gretchen added, There was such disparity between how much time I thought I would have to put into everything. I don't teach any lessons like the ones I taught in college because it's not realistic. I think the workload was misconstrued. While their frustrations and suggestions were genuine, they lightheartedly shared the advice for 
programs preparing future urban teachers that more closely align with the reality of their lived experiences of the first year.

\section{Summary of Frustrations in the Closing Focus Group}

Chayla and Junie's frustrations with gang-related issues their students faced and the collective frustration with feeling ill-prepared for the work of urban teaching are examples of the fifteen examples of frustration present in the closing focus group. These frustrations voiced in the narratives of the closing focus group were noticeably lower than the more than forty narratives of frustration coded in the opening focus group. This may indicate a shift in perceptions regarding the work as viewed through more confident perceptions. While many possible reasons for the shift exist, I suspect teacher participants were gaining additional experiences in the classroom coupled with the support of the induction experiences. This may have contributed to the shift in thinking.

\section{Closing Focus Group: (from) Isolation to Tribe}

This theme is one that emerged as significant because it captured one of the greatest turns teacher participants experienced from the opening focus group, through the induction experiences, and, finally, to the concluding focus group. The teachers spoke candidly about the connections they now feel with one another and as part of the teaching profession. In some ways, they seemed to shift from feeling like outsiders, unworthy of the title of teacher, to a close focus group where they felt they were earning and finding their way. Kay expressed, Meeting everyone here, listening to their experiences, and just realizing that every teacher goes through similar things in urban schools, you come to realize that you're not that bad. Understanding that her struggles were not unique to her and not a reflection of her failures gave Kay confidence that growth was possible and that struggling is part of the process. 
Chayla contributed these thoughts. I have to say that the reason I feel more urban ready now is because I got to talk to other teachers who were like me and I understand that, I'm not abnormal — that my class is not. And that, other teachers have the same issues, and we're all trying our best. Everyone struggles. I think until I met you guys, I didn't really understand that.

\section{Summary of Closing Focus Group (from) Isolation to Tribe}

More than their struggles with the paper workload, more than attempting to manage the severe behaviors of some students while simultaneously attending to other students, more than learning how to navigate their decision-making between the academic and social and emotional needs of students, these teacher participants found comfort in the realization that they are not alone in their struggles. Instead, they seemed to take comfort in knowing that in their journey they were not alone. They learned that while many discouraging events had occurred, they were not condemned to lives of mediocrity in their profession. In the tribe, they had discovered that it is okay to grow into the work and to be patient with themselves as they strived to figure things out. This connection also spurred the turn from feelings of failure and inadequacy to growing confidence.

\section{Closing Focus Group: (from) Inadequacy to Self-Assurance}

The level of self-efficacy in the teacher participants shifted significantly over the course of the study. Some of the early narratives left me wondering whether they would continue through the first semester. Merely listening to their stories troubled me. While there were successes, the level of emotional fatigue left me to wonder whether they would give themselves and students the benefit of learning and growing together. The shifting of their self-efficacy by the closing focus group had me hopeful that they would 
indeed continue to show up and give their all to students. For example, Gretchen and Anne shared insights regarding skills she and other successful urban educators should possess. Gretchen discussed the importance of flexibility and being able to adapt to the wide variety of situations that are going to appear in your classroom and not freezing or panicking or feeling like you can't handle it. I think the longer you teach, the better equipped you are to say, 'Okay, this is something new, but I'm going handle it. That is really important. Anne added, You can never give up because you really don't develop it. In a way, it just happens. So if you give up, you'll never develop it. You just have to keep going back every day, and keep giving it your all. Both Gretchen and Anne shared determined and confident feelings that push them to give it their all, to show up each day without giving up. The teacher participants were beginning to trust that their process of growth was part of a natural progression, not the case of their initial thoughts of personal inadequacy and failures.

The most profound evidence of the evolution of the teacher participants' from inadequacy to self-assurance is found in the closing focus group when I asked once again whether they were urban-ready. In the opening focus group, the only response close to confident was Gretchen's who argued that showing up meant something---it meant she was committed to being there and giving her all. All of the others proclaimed that they were not urban-ready and were not confident that they would ever be ready for the work in their high-need urban schools. Those thoughts changed significantly when asked the same question in our closing session. All six of the teacher participants declared their readiness. Excerpts of their responses are presented here. Gretchen's confidence remained as she stated, I am ready because I go to school every day, and I'm doing what I 
can. I'm trying to get better and, that's it. That's all I can do right now. So as long as I keep doing it, I'm counting myself as ready. Kay added, My original answer was no, but I'm going to say Yes, I am now. I'm not saying I'm a perfect educator, but I'm ready to go in every day, and do what needs to be done on that day. Junie expressed, I am, because I'm motivated to go in, and I want to help these kiddos out. Chayla shared, I originally said No because honestly, I felt inadequate in dealing with all the issues. I didn't have those same issues. But, I think that I am an urban-ready teacher because I understand who my kids are. We're not perfect, by any means, but we are doing the best that we can. Anne contended, The first time I said that you're never ready, you're always learning, you're always growing. You'll never be fully ready to be the best ... perfect, but I do think that I'm giving it my all. I do think that I'm learning and growing every single day. Lola concluded, In the beginning, I definitely didn't think I was ready, but as time has progressed, you have no choice but to be ready. You learn a little bit more each day, becoming more and more prepared. Their thoughts, in their own words, reflect the arc in their thinking.

\section{Summary of Closing Focus Group (from) Inadequacy to Self-Assurance}

From Gretchen and Anne's comments about flexibility and never giving up, to

proud declarations of urban-readiness, we hear the reflective tone of newfound selfefficacies of new urban teachers in high-need schools. Their comments left me encouraged that they might indeed stay to course. At the conclusion of this focus group, I was not concerned that they would join the ranks of new urban teachers who abandon their classes in the middle of the school year. This gave me hope that with the investment of multiple stakeholders, new urban teachers can, indeed, become career educators ready, willing, and able to meet the challenges they may face. 


\section{Summary of Findings}

Figure 5 captures additional thoughts from participants as they reflected during our final session together.


Figure 5: Additional Reflective Thoughts from Participants

The coding of the data collected over the course of the study allowed for a close examination and synthesis of the lived experiences of the new urban teacher participants 
working in high-need schools. Through multiple lenses of analysis, four themes surfaced as significant in their ability to allow insight into their experiences. Those themes are compassion, frustration, isolation that evolved into a sense of tribe, and inadequacy that evolved into self-assurance. The challenges teachers face in high-need urban schools is tough to comprehend if you are not living it each day. For that reason, I felt it was critical to present evidence of these themes through the real experiences and narratives of the urban teacher participants. The goal is not to shock or depress readers, but the goal is to provide honest depictions of their realities and to consider what might be possible when new urban teachers are supported by stakeholders as they are transitioning into their new roles. Providing support for these teachers may reduce the teacher attrition rates in urban school districts and may also improve the school experiences of students and teachers. 


\section{CHAPTER 5: SUMMARY AND DISCUSSION}

"A fundamental step in this challenging of structures is to think about new ways for all education stakeholders-particularly those who are not from the communities in which they teach - to engage with urban youth of color. What new lenses or frameworks can we use to bring white folks who teach in the hood to consider that urban education is more complex than saving students and being a hero?"

Christopher Emdin

\section{Introduction}

In this final chapter, I summarize the study by presenting an overview of the problem that inspired the investigation, revisiting the study questions and summarizing the research design, and presenting a summary of major findings as they relate to current literature. Finally, in the conclusion of the chapter, I discuss implications of the study and recommendations for future studies.

\section{Overview of the Problem}

Kaiser's (2011) study of beginning teacher attrition found that 10 percent of public school teachers who began teaching in 2007-2008 were no longer teaching in 2008-2009. That rate increased as 12 percent of these teachers were no longer teaching in 2009-2010 (Kaiser, 2011). In their review of several studies, Ingersoll, Murrell, and Stuckey (2014) found a 41 percent cumulative attrition rate among new teachers. These numbers shed light on the difficulty high-need schools face in their efforts to hire and 
retain teachers who are able to provide quality instruction to students. For example, Jacob (2007) found that the Chicago public schools receive an average of ten teaching applicants for each available teaching position, yet many of those applicants are interested in working in specific schools—schools they perceive to be appealing, and their desires usually do not include the most challenging schools.

Once hired, maintaining those teachers is a problem. Daukas and White (2010) found that nearly 1,000 teachers leave the profession each school day at a financial and human resources cost that greatly impacts students, especially students in high-need schools. Many educators who do find themselves teaching in high-need urban schools struggle to meet the demands of the classroom without the support and resources they feel are essential to success. In their disaggregation of the data among movers who take on other positions or change locations within the field and leavers who leave the profession completely, Ingersoll et al. (2014) found that those hit hardest by teacher turnover are those serving high-poverty and high-minority students in both urban and rural areas. These and other researchers cited job dissatisfactions related to leadership, salary, voice in decision-making, student behaviors, and lack of resources as reasons teachers used to explain their departures (Helfeldt et al., 2009; Ingersoll et al., 2014; Kukla-Acevedo, 2009). Simon and Johnson (2013) reviewed six recent studies and found that teachers did not attribute factors related to students as the leading cause of their exit. Instead, they found that teachers credited unfavorable working conditions that make teaching and learning difficult for encouraging their departures. These teachers valued social elements of the job such as collegial relationships, healthy school culture, and supportive leadership (Simon \& Johnson, 2013). When these social conditions are absent, 
educators are more likely to leave. Perhaps increased efforts to address the concerns these researchers discovered will slow the mass exodus and encourage more new teachers to seek out and remain committed to working with high-need urban schools. This study was designed to explore ways to support and retain such new teachers.

\section{Summary of the Study}

Researchers have explored the conditions necessary for teachers in hard-to-staff schools to meet with success. Donaldson (2009) purported that multiple stakeholders invest in the support and development of teachers in challenging urban contexts. He posited that both the school district and teacher preparation program must invest in the development of competencies of urban teachers. In an effort further explore the impact of providing new urban teachers with the support of multiple stakeholders, this study used Abawi's (2012) refractive phenomenology methodology as well as Authentic Caring (Valenzuela, 1999), and Community Teacher (Murrell, 2000) theoretical frames to pose the following research questions.

1) What are the lived experiences of first-year urban teachers who participate in collaborative, urban-focused induction experiences?

2) What are first-year urban teachers' perceptions of the impact of their lived experiences in the urban-focused induction experiences on their self-efficacy during the first semester of teaching?

These questions were designed to explore multi-stakeholder support, and these questions extend existing studies by counting new teachers themselves as critical stakeholders responsible for nurturing and facilitating their own success and that of other new urban teachers. 


\section{Research Design Summary}

This study was conducted in a large, urban school district situated in the southeastern part of the United States. The school district serves as the public educational institution for near 100,000 kindergarten through grade 12 students annually. In 2017-18, the district fell short of meeting the state goal for annual yearly progress and was designated as needs improvement by the state board of education. Six first-year urban educators participated in the study during the 2017-18 school year. Each teacher was working in a school identified by the state as needs improvement. One of the three schools where teacher participants were working was a state focus school which means that state level supports were present and guiding the leadership within the school. The five-week study provided participants with one Saturday, six-hour professional experience at the start of the study. There were three two-hour subsequent professional learning cohort experiences. Finally, there was a closing six-hour professional learning and conversation session to conclude the study.

\section{Major Findings of the Study}

All six of the study participants completed the school year in the same position they held during the study. At the time of publication of this study, four of the six teachers were still teaching in the same high-need school where they were teaching during the year of the study. While all expressed deep concerns about their working conditions during the study, all participants remain committed to growth and development as urban educators as evident in their continued employment at their current schools. Some have reached out to reconnect with me for varied reasons as well. These educators who continue working in high-need schools give me hope that with care, these educators may become career educators. 


\section{Overview of Findings}

Through this study, I have posited that new urban teachers in high-need schools must not just be pedagogically ready to teacher, but they must also be urban-ready or at least willing and able to position themselves to develop urban-readiness or competence. Over the course of the study, four theme surfaced. The four themes that best capture the lived experiences of teacher participants are compassion, frustration, isolation that evolved into a sense of tribe, and inadequacy that began to grow into self-assurance. These themes provide significant details of participants' lived experiences and how their experiences evolved as they engaged in the work of the study. Their compassion for teaching was evident from the start, but their compassion for students as individuals and for the work of being urban teachers grew. Their frustrations remained a constant throughout the study but narrowed in their intensity and frequency within their narratives as the study progressed. Their frustrations focused less on student behaviors and focused more on systemic issues with their preparation programs. The feelings of isolation were greatest at the start of the study, but those feelings of isolation began to fade as the camaraderie and kinship within the group increased. Finally, feelings of inadequacy for the work were pronounced at the onset of the study but lessened significantly as teacher participants began to see value in their personal and collective struggles. Each of these themes serve to give us insight into the realities these six new urban educators faced in their high-need schools and how the support they received from invested stakeholders and provided to one another seemed to help improved their self-efficacy in the work.

\section{Conceptual Framework: Authentically Caring}

Valenzuela (1999) found that the relationships teachers foster with students in the context of schools either add to or subtract to the academic experiences of students. 
When students feel their teachers care about them as people before focusing on content, students feel valued and are more successful in school. On the other hand, those who feel their teachers focus almost exclusively on content lessen their ability to be successful in school. None of the teacher-participants in this study boasted of giftedness as urbancompetent educators. In fact, their brutal honesty about their competence over the course of the study illuminated their understanding of the need to grow. In spite of sometimes seemingly harsh criticisms of self, the teacher participants were service-oriented and believed a huge part of their job to be caring for their students. Authentic caring rooted them in much of the work in spite of their self-disclosed shortcomings.

New teachers are often especially focused on their purpose of employmentteaching content in the name educating students. Some teachers may lock their focus on this mission without considering the whole child. These teacher participants were working with students who required a focus beyond the academics. As a result, the teachers approached the work from a caring place. They were able to sympathize with students, some of whom faced life circumstances that the teachers, and most others, would find unfathomable. They also understood the potential impact of a quality education on the lives of students. It is through a caring heart that they approached their work to find balance between authentically caring for students as whole people and for their specific academic nourishment. Just as Valenzuela (1999) suggests, teacher participants seemed astute to the reality that their interactions with students were critical to student success. Their realizations of the magnitude of the non-academic elements of the work were jarring, panic-inducing, and filled them with self-doubt at times. In the face of the challenges, each participant found their work meaningful and critical. 
Further, participants avoided false empathy that positions a person to convince themselves and others that they know all about the challenges other people face. In this perceived understanding, some act in ways that further perpetuate the conditions marginalized populations face (Delgado, 1996; Warren \& Hotchkins, 2015). Instead, teacher participants cared for students with whom some of the teachers became genuinely empathetic as they discovered that they shared teacher-student life experience intersections that they had not considered before. For example, Junie, Kay, and others realized just how connected they were with their students when exploring adverse childhood experiences. Teacher participants identified with the impact of loss, divorce, and incarcerated parents. Upon the realizations that they were more like their students than different, teacher participants were genuinely empathetic about the adversities their students faced. They realized that if they were able to become successful as college graduates and educators, their students were not doomed to non-productive lives. Instead, success and happiness were possible. This shift encouraged some participants to look at students as though they were still on a trajectory toward life success in spite of obstacles. When one can not only sympathize, but empathize with the journey of another, we are better positioned to operate from a space of authentic caring. Teacher participants in this study found that space.

Each participant demonstrated an understanding that they must authentically care for students and that misguided care could subtract from the learning and lives of students just as Valenzuela (1999) theorized in her Authentic Caring contextual framework. Teachers who care authentically are also aware of the need to connect with the communities they serve. Several times over the course of the study, teacher participants 
were torn between protocols and perceived concepts of right and wrong and what their hearts were feeling for students they authentically care about. For example, Gretchen's conflicted reflections about what is truly right and wrong when she began to fully consider the scope of the challenges her students' families faced in an effort to provide the best normal they could manage for their children. She and other participants came to realize that things are not always what they seem and that when they consider circumstances and authentically care for their students and families, they are better teachers for their students and, in some cases, better people. When teachers genuinely care for their students, they recognize that they must care for their students as people, first and foremost. They are then able to bring the academic and personal lives of students together in ways that add to, not distract from, the school experience.

\section{Conceptual Framework: Community Teacher}

Community teachers are invested in the communities where they work. These teachers feel called to serve with the goal of elevating the lives of students. Many of them have attended area schools. They have knowledge of the culture of the community, and they use what they know to bolster their effectiveness with a diverse population (Murrell, 2000). While the teacher participants of this study do not perfectly meet Murrell's description of community teacher, the evidence of each of the participants' pursuit of these traits became increasingly evident over the course of the study. The most evident of Murrell's traits was feeling that one is called to serve as an educator. All six participants expressed the pull to serve and nurture the development of the underserved in the community. Other traits emerged and began to grow over the span of the study.

One area participants noted as a needed focus was knowledge of the community they are serving. Though not well-versed in the assets and resources within the 
neighborhoods initially, teachers worked to learn more. The focus school system maintains a student assignment plan that attempts to ensure diversity through a bussing system that moves students within and across neighborhoods. This means the student body of each school was made up of students and families from across the large urban area. Students arrived to school in a variety of ways-walking, riding in vehicles, or riding in buses. So, even though they did not live in the neighborhoods where their schools were located, participants worked to develop relationships with the school community. They began to realize that there are assets within the neighborhoods and throughout the community that could, in reciprocal ways, support one another to the benefit of the whole child. They started connecting with neighborhood resources to support the needs of their families. For example, Gretchen's efforts to help families connect with child care services and Lola ensuring families were connected with programs that send students home with groceries over the weekend to ensure they are able to eat while away from school.

Teacher participants were also intentional in their efforts to enhance their cultural competence by seeking to understand the cultures of the school community. They sympathized, and in some cases, empathize with the realities some families faced. Their reflections showed their keen understanding that success on the academics within schools was only possible if connections were established with students beyond academics. The planning for and delivery of culturally sensitive texts in Kay's unit on diverse families, Junie's trip to the used bookstore to find diverse texts that students could connect with, and Chayla's reflection on the diverse lives within a single culture as she noticed distinct differences within the African-American culture are examples of these teachers' 
commitment to inclusive classrooms where cultures were celebrated and leveraged for teaching and learning, not feared.

Participants had not solidified all of the characteristics of Murrell's (2000) community teacher framework. However, they were developing as educators who felt called to serve an underserved community, who were working to connect with the community, and who sought to capitalize on the culture and other assets of the community to the advantage of the students and the community as a whole just as Murrell suggests. Reflective practices and self-awareness as they continue to grow as new urban educators may move them to continue efforts to act in community conscious ways where they value the assets within the community or funds of knowledge they bring (Moje et al., 2004; Moll et al., 1992). This may include connecting with local churches, community centers, and other neighborhood organizations to support one another in efforts to serve and support families.

\section{Major Findings}

The following major findings and conclusions of the study are discussed as they relate to current literature:

\section{Major Finding Number One: Compassion}

Compassion drives many of the thoughts and actions of new urban educators.

Conclusion. Urban educators in high-need schools sometimes face high-poverty, diverse, traumatized students among their student population. These students have needs of schools that extend beyond academic nourishment. They require nurturing of the whole child. They need teachers who authentically care for them as people, not just vessels into which knowledge is distributed by enlightened teachers. Valenzuela's (1999) Authentic Caring frame was evident in this study. Teachers set aside the pursuit of 
educating students when there was a need to care for the individual. Their hearts and the genuine ways they cared for students were evident in their reflections. When Junie and Chayla recognized the implications of children who find it difficult to sleep due to unpredictable and sometime unsafe sleeping conditions, instructional content took a backseat. Gretchen gingerly approached the conditions that led to a call to both the police and Child Protective Services but recognized that situations are not just clear-cut or black and white. Junie and Kay connected with students in new ways as they realized they had common traumatic life intersections that brought them to tears. Compassion led participants to see that there are conditions and circumstances that require heart-led responses and interactions.

The teacher participants were compassionate as new urban educators. Their actions align with Äärelä, Määttä, and Uusiautti (2016) who conducted a qualitative study of high-need students who in their teen years found themselves incarcerated in the country of Finland. These researchers sought to discover the attributes their participants felt were critical for caring educators. Participants suggested the following ten caring teacher qualities. They include the following: 1) they are present; 2) they listen; 3) they thank others; 4) they work collaboratively with families; 5) they teach everyone, not just the easy ones; 6) they find enjoyment in the work; 7) they forgive when mistakes or bad choices are made; 8) they welcome students back to school and express care when they are absent; 9) they are courageous enough to intervene in bullying incidences, and 10) they take care of themselves (Äärelä et al, 2016). By authentically caring, the teacher participants in this study were also present as Lola was drawn to work on a day when taking the off was justifiable. Participants showed kindness through de-escalating 
interactions with students who were on tirades that destroyed a classroom or subjected them to being spat on as Lola presented in her PhotoVoice entries. They truly listened to connect beyond the surface with students and families in an effort to meet the needs of the whole child. The participants in this research study demonstrated compassion throughout the study as they authentically cared for students.

\section{Major Finding Number Two: Frustrations}

Frustrations run high with new urban educators, but these frustrations decrease when the new teachers are supported as they learn and grow.

Conclusion. Teachers in hard-to-staff schools with populations that include students of greater than average socioeconomic disadvantages, lower student achievement, often lesser sought after geographic school location, and challenging teaching conditions are faring worse. In fact, fifty percent of teachers depart these hardto-staff schools within the first five years by way of resignations, transfers or retirement (Ingersoll \& Smith, 2003; Morgan \& Kritsonis, 2008). Compiling the challenges is the pattern of the most skilled teachers opting to transfer from high-need, urban schools to schools they anticipate will be less challenging (Costelloe, 2006; Simon \& Johnson, 2015). Many teachers in high-need schools may find themselves frustrated by their working conditions. This theme was evident in the teacher participants of this study.

Teacher participants were often frustrated for a variety of reasons. The workload was taxing. The PhotoVoice images of piles of paperwork and departures in the dark of night captured their frustrations with the elusiveness of work-life balance. The student behaviors were also a concern. The PhotoVoice images of the teacher's coat who had been spat upon by a student and the collage of broken pencils and toppled chairs capture their frustrations with student behaviors. There was also frustration with their teacher 
preparation programs regarding their perceived readiness for the work seemingly inherent with high-need schools as documented in their discussion during the closing focus group.

The frustrations of this study's participants align with what researchers are finding. Teachers are leaving the profession at alarming rates largely because of frustration with working condition that include but extend far beyond student behaviors (Helfeldt et al., 2009; Ingersoll et al., 2014; Kukla-Acevedo, 2009; Simon and Johnson, 2013). Daukas and White (2010) compiled five factors that positively impact teacher retention. Among them are some that are evident in this study's participants' frustrations. According to Daukas and White (2010), teachers are more likely to stay in the profession if they are able to 1) collaborate with colleagues to engage in professional works; 2) engage in professional learning experiences; 3) have autonomous experiences; 4) have productive interactions with supportive leadership; and 5) have some voice in the facilitation of student learning. The participants grew less frustrated as the study progressed as they collaborated with colleagues and engaged in professional learning that was custom designed to support new urban teachers in high-need schools. Perhaps this level of support will encourage their retention beyond current trends for new urban educators.

\section{Major Finding Number Three: Isolation}

Isolation that is common among new urban educators in high-need schools

decreases when new urban teachers connect in non-evaluative ways with a collaborative network of support.

Conclusion. New teachers often experience isolation which may cause them to feel uneasy, lonely, and inadequate (Prilleltensky, Neff, \& Bessell, 2016). This stress challenges their ability to learn and grow in favorable conditions. This current study 
provided a protective factors that Prilleltensky et al.(2016) found to counter isolation. This study was set up to provide a network of support for these new urban educators that served to combat some of their feelings of isolation. They were, instead, a collective, not easily broken by the challenges of first year teaching in high-need urban schools.

Initially, participants in this study expressed feeling as though they were alone on their journey as first year teachers. They felt their struggles were unique and singlehandedly reflected their worth as educators. For example, Junie beautifully expressed the transformation of mindset she underwent when she wrote of how she had first resisted and bemoaned her position as an urban educator. While participating in the study, she came to realize her potential as a qualified urban educator who had room to grow but also gifts to share. Not unlike participants in this study, Charner-Laird, Szczesiul, Kirkpatrick, Gordon, and Watson (2016) found in their study of 17 novice teachers that isolated teachers felt as though they were on their own, unprepared to be effective in their work, and were discouraged about the realities of the job. These same researchers found that novice teachers found collegial interactions to be supportive even when not directly linked to critical discourse (Charner-Laird et al., 2016).

Ostovar-Nameghi and Sheikhahmadi (2016) reviewed research on teacher isolation and the impact of collaboration. They posited that collaboration is essential to teacher learning. They further found that push-in and top-down professional development are no longer the standard. Instead, professional development involves teachers collaborating to learn from one another's experiences in exercises of reciprocity where practice, not theory, improves practice. They also cautioned that these collegial interactions or collaborative experiences should not be forced as such efforts may lead to 
artificial congeniality (Ostovar-Nameghi \& Sheikhahmadi, 2016). Participants in this current study found benefit in connecting with one another as new urban educators and in the company of others who are willing to invest in their success. Their learning experiences through PhotoVoice and other professional conversations provided opportunities for the group to learn from one another in practical, not theoretical ways. Participants were both the beneficiaries of the experiences of others, and they were the facilitators of the learning of others as they both learned from and shared their experiences.

Context-based, personalized learning is found in the situated cognitive theory that Bowe, Desjardins, Covington-Clarkson and Lawrenz (2017) describe as the concept that social and environmental factors within a learning environment are critical components of the learning processes as they directly impact how well one learns. In other words, context matters. Meeting new teachers where they are with relevant, context-specific professional growth and development are endorsed by the Every Study Succeeds Act (ESSA) of 2015 just as the situated cognitive theory purports. This study provided new urban educators with this type of context-specific, situative learning. Such supports work to decrease feelings of frustration and isolation, and they are designed to increase teacher self-efficacy.

The research literature of Oakes et al. (2002) influenced the findings. These researchers assert that novice educators are able to learn and grow when 1) engaged with one another and experts on tasks that are meaningful to all, 2) part of a group allowing for the development of broader collective knowledge because of multiple perspectives and ideas, 3) engaged in dialogue especially around artifacts where they are challenged to 
argue and defend their positions as well as entertain the ways others see things, 4) developing emerging identities as knowledge around the work increases and evolves, and 5) mutually engaged in common tasks that allow them to develop socially accepted knowledge that spurs further learning. These principles are relevant to the teacher participants of this study who found kinship as they engaged in experiences as a collective, shared learning and meaning-marking around artifacts in the PhotoVoice experience, and experienced emerging identities as they considered the personal urban competence.

Over the course of the study, teacher participants who were brought together by a common context confided in and leaned on one another for strength on their journeys. The simple conception that we are stronger and smarter together than on our own aligns with the work of Bandura (1997) who introduced the notion of collective self-efficacy as the theory that a collective can gather, organize, and act in ways that lead to the successful execution of common goals. Likewise, Bandura's work aligns closely with the Kenyan proverb where the sticks provide collective strength that eludes each when working independently. Simply, there is strength in numbers.

\section{Major Finding Number Four: Inadequacy}

Feelings of Inadequacy among new urban educators in high-need schools decrease and collective and personal self-efficacy increase when growth is nurtured in a risk-supported environment.

Conclusion. Those who feel isolated may also feel ineffective or inadequate in their work. Teacher participants in this study frequently questioned their worth as educators. One such example within this study includes Kay and Lola who, with a racial and cultural background similar to many of their students, found themselves surprised by 
the challenges they faced with a largely African American population. They felt the cultural prior life experiences should have carried influence in the classroom. When they discovered this was not the case, they questioned their capacity or personal proficiency as urban educators. Prilleltensky et al.(2016) found that feelings of inadequacy was another personal risk factor that new teachers face and suggested professional development and non-evaluative mentoring experiences as protective factors to counter those feelings.

Bandura's (1989) social cognitive theory speaks to personal agency one accesses to influence life factors. This theory asserts that one contributes to their own circumstances in self-elevating or self-impeding ways (Bandura, 1989; Schunk \& DiBenedetto, 2016). Teacher participants in this study began to increase their selfefficacy as their understanding of personal agency developed. In the opening and closing discussions regarding their personal urban competency, there was an evolution of thought. Teacher participants moved from almost exclusively claiming their lack of urban-readiness to a positioning of greater agency and self-assuredness in their practices as urban educators. Each were humble enough to not claim expertise, but they grew in their ownership of a personal ability to influence that urban competence. This image provides a visual that captures the evolution of themes over the course of the study. 


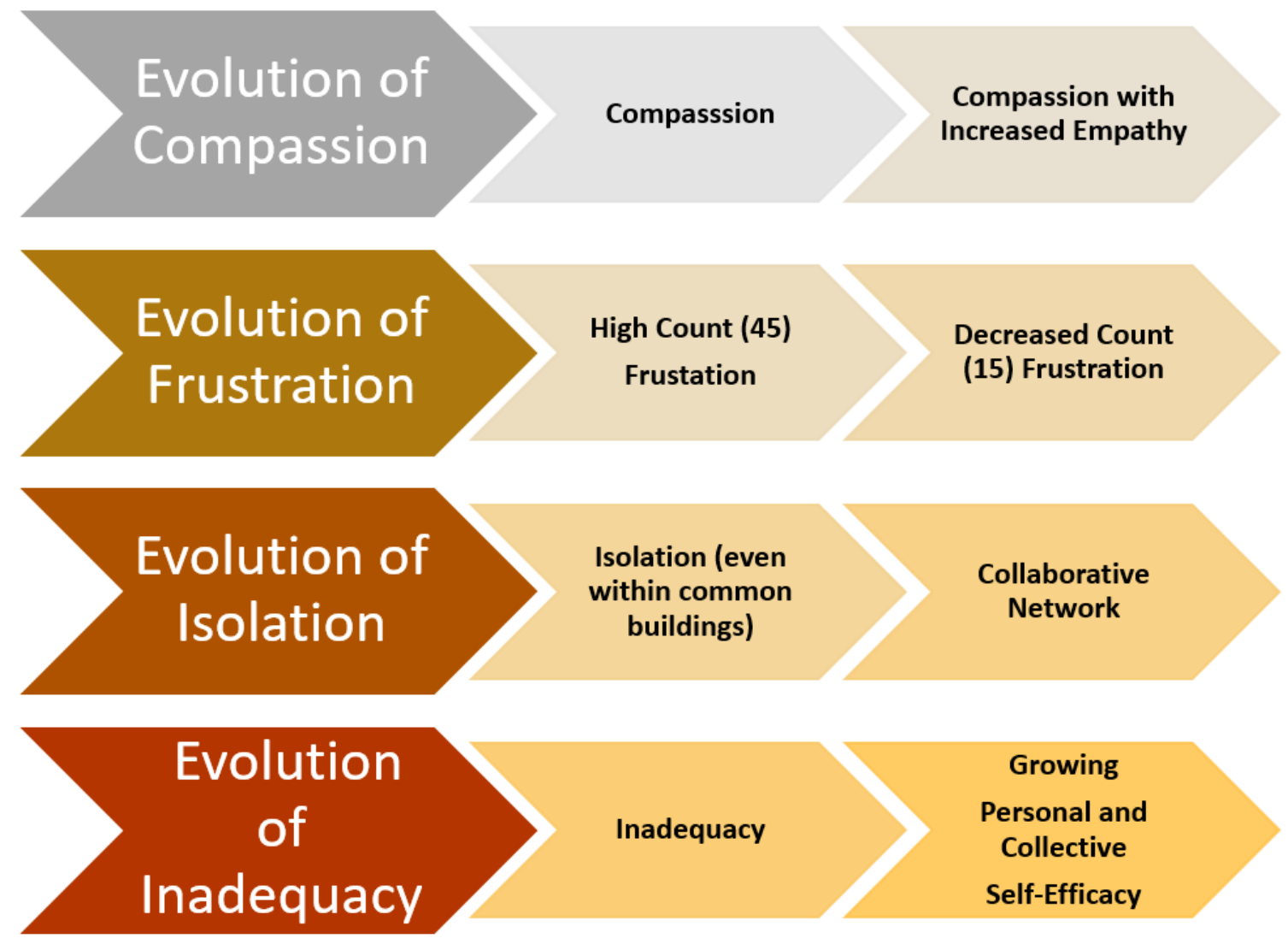

Figure 6: Evolution of Themes across Major Findings

\section{Conclusions of Major Findings of the Study}

The outcomes of this study examining the lived experiences of new urban teachers in high-need schools indicate that collaborative supports from invested stakeholders have the potential to influence the retention rates. In this study, new urban teachers demonstrated great compassion as they demonstrated authentic care for students, experienced great frustrations related to the workload, felt isolated in their journey as new urban educators, and experienced feelings of inadequacy in the work. Over the course of the study, as supports were provided from multiple stakeholders representing the local university, school district, and teacher participants themselves, compassion was evident, frustrations deceased, isolation turned to collective self-efficacy, and inadequacy began to wane as self-confidence grew. These themes, and the way they evolved with supports in 
place, may indicate that there is potential to positively influence the experiences of new urban educators. Stakeholders have the potential to impact the longevity of new urban educators. These findings are supported by prior research by Donaldson (2009) who called for new teacher supports from multiple stakeholders.

\section{Discussion of Implications}

In the previous section, the major findings of the study were discussed in the context of current literature. The following section discusses the implications for policy makers, teacher preparation programs, urban school districts, and prospective urban educators. This graphic captures the collaborative way I recommend the stakeholders collaboratively support new urban educators.

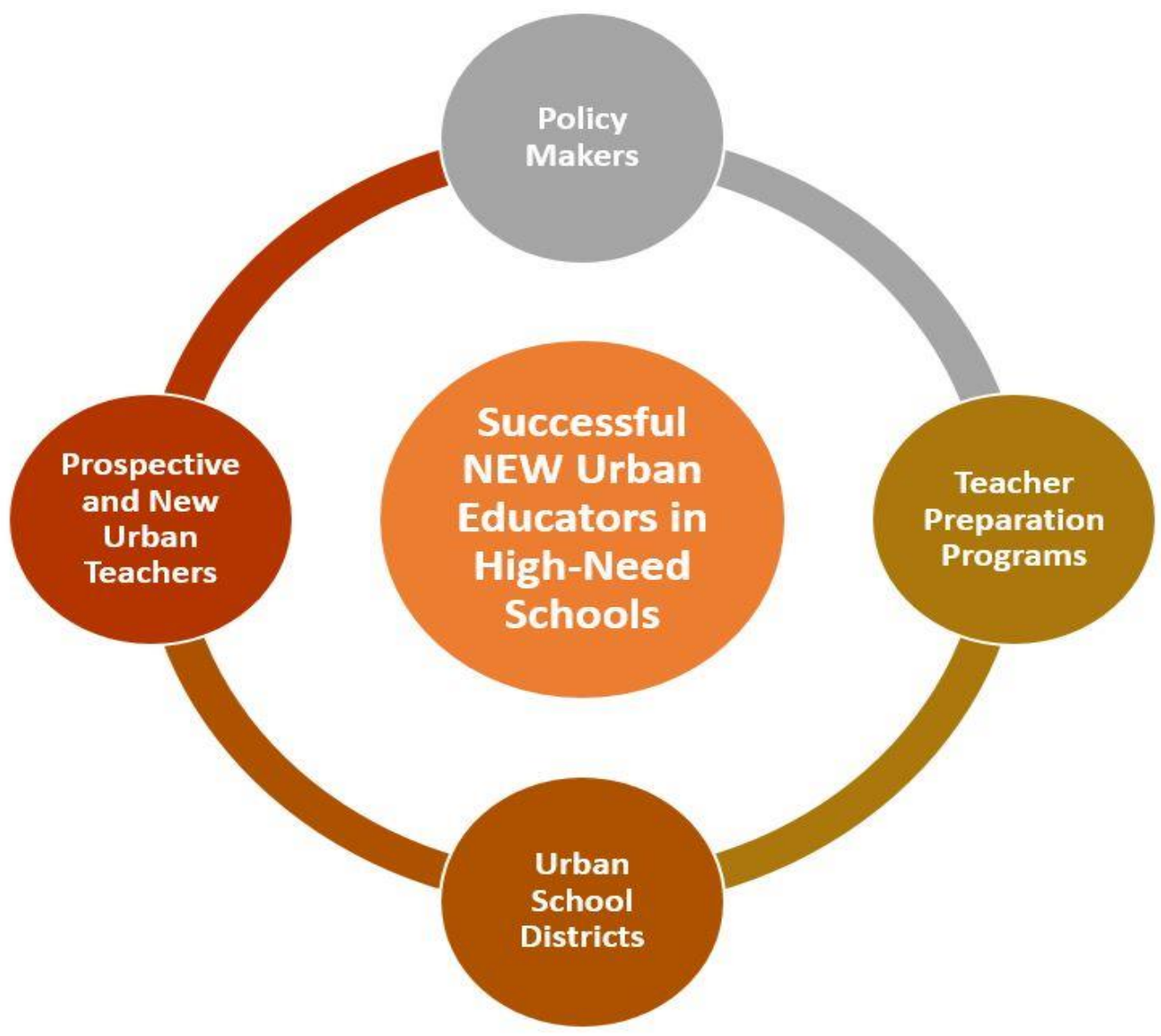

Figure 7: Implications for Major Stakeholders 


\section{Implications for Policy Makers}

Educational policy is of great interest to varied members of our society with many politicians, business leaders, and media outlets classifying public education to be in a state of crisis (Fowler, 2013). Their declarations regarding the state of public education give rise to an influx of policies, developed many times in the absences of some of the people most invested in public education, the educators. "State governments have asserted their authority over public schools by issuing a bewildering array of new policies and policy proposals. More often than not, they did not ask public school educators for input" (Fowler, 2013, p. 9). Educators are often, instead, considered a source of the problem and, as a result, are not treated as knowledgeable and valued stakeholders. Bypassing educators, policymakers consult business leaders and researchers (Fowler, 2013). I contend that studies like the one I have conducted and many others bear witness to the importance of inviting those intimately engaged in the work of education to be meaningful contributors to the policy development process at the federal, state, and local levels. This is a review of policies related to new teacher supports.

The federal policy, Every Student Succeeds Act (ESSA) of 2015 calls on the field of education to look upon teacher professional growth and evaluation differently. In fact, in the regulations under TITLE II-PREPARING, TRAINING, AND RECRUITING HIGH-QUALITY TEACHERS, PRINCIPALS, OR OTHER SCHOOL LEADERS, Section 2001 includes these purposes “(1) improving the quality and effectiveness of teachers, principals, and other school leaders; (2) increasing the number of teachers, principals, and other school leaders who are effective in improving student academic achievement in schools; and (3) providing low-income and minority students greater access to effective teachers, principals, and other school leaders" (ESSA, 2015, p. 113). 
These explicit resolves require shifts in the ways in which universities prepare teachers and the school leaders who guide them. They also call upon those principals to be more invested as instructional leaders who help teachers navigate professional growth and development for the benefit of students. Just as education has long extoled differentiation and responsive teaching as best practices for students, the professional growth of teachers, as described by ESSA, should involve differentiation and responsive leadership for those guiding teachers. Since this is the case, induction experiences for new teachers, especially new urban teachers, should be responsive in nature, meeting the needs of these new educators in responsive and differentiated ways. Experiences, like those facilitated in this study, designed to meet the specific needs of teachers are outlined in the language of ESSA and should guide state and local decision-making about the preparation and training of educators such as the new teachers of this study.

\section{Implications for Teacher Preparation Programs}

Some teacher preparation programs are working to update their programs and practices to prepare students for the realities of urban classrooms. Berghoff, Blackwell, and Wisehart (2011) worked to be responsive to the needs of their students who they had taught strong instructional practices in their coursework. The researchers were disheartened when their students quickly yielded to norms of urban school that they had explicitly worked to combat like institutional racism, teaching to standardized tests, and lock-step curriculum. They found their students falling in line with business as usual in their urban school placements. In response, Berghoff et al. (2011) focused on critical reflection where their students were called upon to question the ways in which they further fueled inequitable schooling. By engaging in critical reflection, their students began to challenge their personally biased assumptions to find ways to foster student 
success instead of blaming those challenges on families or student limitations (Berghoff et al., 2011). Teacher preparation programs must be responsive to the needs of student graduates. For those universities geographically located in urban areas, it is critical that their teacher preparation program provides future teachers with the skills, strategies, and mindsets necessary to match the needs of the population.

Teacher preparation programs would benefit from establishing a clear vision and mission. Doing so will allow more successful execution of responsive teaching practices that prepare future teachers to meet the vision. Cochran-Smith, Villegas, Abrams, Chavez-Moreno, Mills, and Stern (2015) extensively reviewed teacher preparation program research studies where they identified four main categories of teacher preparation research addressing diversity and equity. Those categories explored 1) methods programs use to prepare their students to teach students from diverse backgrounds, 2) efforts at recruiting and developing a diverse teaching workforce, 3) deep analyses of the pedagogies, content, and structures of programs designed to prepare teachers for diverse student populations, and 4) studies that examine the experiences and expertise of teacher educators for preparing their students for diverse student populations (Cochran-Smith et al., 2015). This review of existing studies on the preparation of teachers for diversity and equity speaks to the efforts preparation programs are making to meet the needs of their students. These and other categories of studies will remain essential if education is to hope to create systems that allow all students the benefit of a learning environment where they can and do succeed.

\section{Implications for Urban School Districts}

Public education provides structures that systemically avoid assigning students to schools in ways that creates microcosms of society in each school. Instead, there are 
schools that are overpopulated by those of privilege, others that are overrepresented by those who are marginalized, and other schools that lie somewhere in between. These structures tilt the challenge scale sharply to the disadvantage of those already disadvantaged by creating learning and work conditions that new teachers often find challenging. These high-need, hard-to-staff (Morgan \& Kritsonic, 2008) schools created by student assignment plans approved by the district, place greater burden on high-need schools as they struggle to hire and maintain qualified teachers. Barring a balancing of student assignment plans, urban school districts must provide the supports needed in high-need schools. Those needs include human, financial, and professional resources.

One way to nurture the development of urban competence in new urban educators is by strategically planning support. I have drafted a plan of action (see Appendix I) that urban school districts might facilitate. This plan is designed to support their new urban teachers of that district as they begin their careers as urban educators. It includes a yearlong support system orchestrated by the district but inclusive of multiple stakeholders. The cohort-based plan includes professional development and also positions new teachers as action researchers who are empowered to positively address problems within their practices. Their year-long action research projects culminate in a community showcase of urban teaching and learning. There is also a component that allows for "graduates" of the yearlong induction experience to return in subsequent years to support the on-boarding of new urban educators who come after them. The goal is to build a system of support that sustains new teachers as they join the profession and to help the school district to ensure its students have teachers who are ready, willing, and able to 
learn and grow into career educators in urban districts. Implementing a system of support such as this one may encourage urban teacher retention.

\section{Implications for Prospective and New Urban Educators}

\section{Developing Urban Competence}

Authentically caring for their students and increasing their efforts to become community teachers helped to provide teacher participants in the current study with direction in the sometimes chaotic moments of their first year. They were beginning to increase their overall urban competence - culturally competent, poverty-informed, community-connected, and trauma-informed. Based on the findings of this study, I suggest prospective and new urban educators unearth and actively seek to nurture two characteristics that might prove essential to their success in high-need urban schools. Those characteristics are 1) connections - with students, families, the urban community, and a professional network, and 2) critical reflection - as part of a professional collective and as individuals. Being proactive in seeking to nurture urban competence may increase new urban teachers' self-efficacy and encourage greater teacher retention.

\section{Connections with Students, Families, Community, and a Professional Tribe}

Urban-Competent Educators unearth and build on authentic connections with students, families, the urban community, and a professional tribe of colleagues by drawing on commonalities instead of distancing themselves based on what may be outward obvious differences. Urban-Competent Educators resist urges to distance themselves from the lives, culture, and history of their students. Instead, they seek ways to establish deep meaningful bonds with their students and families that unify school, home, and community. They understand the importance of connecting and seek to make the work of schooling, challenging content, implications of poverty, explosive episodes, 
or life traumas more bearable. These educators find richness in celebrating ways their lives are connected with the lives of their students. They embrace complex realizations that they may have in common with their students such as divorced or absent parents just as Junie and Kay came to see in the study. The urban teacher's shared ACEs and subsequent resilience may serve as a model of possibilities for a student who is struggling. The connections with students and families matter to the urban-competent new educator.

Urban-competent new educators are firm believers that there are assets within and across urban communities that are to be celebrated and that add value to the academic experiences of school for both students and teachers. They understand that it is not possible to separate students' personal lives from that of their school experiences. Given this reality, these educators do not criticize or bemoan the woes of urban communities. Instead, they seek to capitalize on the strengths of the urban community. They use things like rhythms and hip-hop that are loved by so many of their students to access content by singing or rapping everything from the alphabet to the original thirteen colonies. They connect with community resources and organizations to fill voids in the lives of students who need them most. They see value in assets of the community and avoid perpetuating highly publicized negative stereotypes of the community. They realize that attempts to squelch everything students value is detrimental to the educational process and to building relationships. They intentionally seek opportunities to capitalize on cohesion between school experiences and the outside lives of students. They believe that school experiences can and should add value to the lives of students without devaluing that which matters most to students. 
Urban-competent new educators also value connections with a professional tribe that both feeds their need for support and allows them to serve as contributing members of the collective strength simultaneously. Whether formally sanctioned or informal team meetings, at their personal requests of others, or organically evolving, the urbancompetent educator sees value in and finds ways to connect with others. Important in this tribe relationship is not only their ability to receive timely support and encouragement when the work is really hard, but equally important is their need to feel that they are contributing members of a community that is made better by their involvement within it. They fully subscribe to the Kenyan proverb, sticks in a bundle cannot be, or at least are not easily broken, so they both gain strength from and provide strength for their tribe.

New urban educations are better served by actively nurturing connections with students, families, the urban community, and a professional community. By building relationships, they are better positioned to create and nurture a learning space the values the lives of students, their families, the community, and the urban teacher while advancing academics.

\section{Critical Reflection}

Urban-competent educators are well-versed in their content and are able to access best practice strategies to appropriately meet the needs of students. Oakes et al. (2002) posited that proficient urban educators are effective in connecting with students while likewise effective in delivering quality instruction. New urban teachers must manage their growth in both key areas simultaneously. Critical reflection as part of a professional network is one key to the development of aptitude for delivering quality instruction. New urban educators could benefit from critical analysis of practices as part of a professional network (Coffey, 2009). Like the teachers of this current study who were often simply 
trying to gain professional orientation, new urban educators may find this critical reflection most beneficial in the company of other educators where they receive guidance from more experienced educators while they simultaneously contribute to the growth of others.

Urban-competent educators are in constant pursuit of a deep understanding of the content they are charged with delivering and the most effective methods of delivery according to sometimes widely varying academic and emotional needs. The management of this instructional charge challenges many experienced educators with a diverse toolbox of strategies. For new urban educators in high-need schools, this challenge may seem insurmountable. However, effective urban educators tap into collective and personal selfefficacy that encourages them to be resourceful and creative in a quest for sound instruction that best meets the needs of their students. Paired with other characteristics, especially connections with students, families, community, and a professional tribe, content clarity increases as does the overall competence of urban educators.

Personal critical reflection is equally important. New urban educators must take time to critically examine their mindset, focus, motives, and growth in the work. It is through regular check-in with their positioning and how it impacts their actions and reactions that they are able to increase their urban competence. Chayla's reflection on how she responded to non-conforming students allowed her to see how her actions escalated the situation. In her critical reflections, she consider how slightly different actions in the moment might have quickly de-escalated or made non-issues of minor infractions in the hallway. So, engaging in professional discourse and critical reflection 
with others and personal critical reflection may increase the competence of urban educators.

\section{Study Limitations}

This study was not without limitations. While there are challenges in high-need middle and high schools, this study focused exclusively on new urban teachers at the elementary level. The needs of middle and high school teachers may differ from those of elementary educators, and this indicates that their experiences are worth of study. Another limitation of the study is the absence of representation from male and other minority educators. Their voices may represent ideas not evident in the data collected from the participants of the study. Finally, the length of the study may represent a limitation. A lengthier window of support would likely have a greater impact on new teachers. The induction plan presented in Appendix J captures my ideas regarding how these supports may be implemented across a full year and beyond to increase the retention of new urban teachers. Each of these limitations offers opportunities for further exploration of the collaborative supports stakeholders may provide for new teachers.

\section{Summary of Implications}

Policy makers, universities, school districts, and new urban educators have the potential and power to positively impact student learning when those students are in highneed schools with new urban educators. Policy makers can mandate structures that support these new teachers and provide the funding for supports. Universities can commit to being responsive to the needs of the students that their students will ultimately serve in the urban classrooms. Teacher educators can ensure future teachers have the tools and experiences that align with the needs of the community the future teachers will ultimately serve. Urban school districts can provide the human and financial resources new teachers 
need to be successful. Implementing a strategic plan for on-boarding new urban teachers may positively influence retention. Finally, new urban teachers should work to intentionally connect with students, family, the urban community, and a professional support system as they seek to build urban competence. They should also engage in frequent critical reflection that increases their awareness of how their actions and reactions impact their personal and professional learning climates. With awareness and intention, all of these stakeholders can positively impact the learning conditions of all students in any classroom, especially students in our high-need urban classrooms.

\section{Recommendation for Future Research}

In this study, I focused on key stakeholders in urban education that include universities, school districts, and new urban educators. I utilized resources from the university, school district, local community, and the new urban teachers themselves to support these new urban educators. However, there are other stakeholders worth exploring in future research. They include, but are not limited to, parents of students in high-need urban schools, students in high-need urban schools, and administrators in highneed schools. By exploring these other stakeholders, districts may be able to establish stronger support systems for new teachers who, when at their best, are better educators for children within these schools.

I contend that we should continue to explore innovative, yet cost effective ways to increase the urban-readiness of teacher candidates prior to and within the first year of their teaching positions to increase their staying power as successful urban educators. I postulate that an induction experience facilitated and managed by the collaborative efforts of both urban teacher preparation universities and the urban school system is essential to the successful induction of new teachers in the urban classroom. Critical in 
this experience is the need to explore the new teachers' investment in their own growth and development of urban competencies. Teacher preparation programs must be on the pulse of the needs of teachers entering urban classrooms and, beyond pedagogy, provide them with the tools essential for success in often challenging urban schools. In turn, the school district must own their part in the state of affairs in their schools and classroom by doing their part to ensure those they hire are mentored in risk-supported ways as they transition into the classroom. Strategically exploring ideas like these may help to address the growing teacher recruitment, retention, and turnover challenges faced both across the nation and within the researched large urban school district. I believe supporting new urban teachers to be urban-ready, willing, and able will increase teacher staying power and decrease teacher attrition, especially in our high-need hard-to-staff urban schools where urban diamonds can sparkle with brilliance as well. 


\section{REFERENCES}

Äärelä, T., Määttä, K., \& Uusiautti, S. (2016, January). Caring Teachers' Ten Dos. In International Forum of Teaching \& Studies (Vol. 12, No. 1).

Abawi, L. (2012). Introducing refractive phenomenology. International Journal of Multiple Research Approaches, 6(2), 141-149.

Abawi, L. (2013). Shared pedagogical understandings: Schoolwide inclusion practices supporting learner needs. Improving Schools, 16(2), 159-174.

Abawi, L. (2015). Inclusion 'from the gate in': Wrapping students with personalised learning support. International Journal of Pedagogies \& Learning, 10(1), 47-61.

Abdul-Karim, S. (2017, Feb 11). New haven psychologist addresses urban trauma; challenge blacks to seek mental health professionals. New Haven Register Retrieved from http://echo.louisville.edu/login?url=https://searchproquest-com.echo.louisville.edu/docview/1867358569?accountid=1466

Axworthy, L., DeRiviere, L., \& Rattray, J. M. (2016). Community learning and university policy: An inner-city university goes back to school. International Indigenous Policy Journal, 7(2), 1-20.

Bandura, A. (1997). Self-efficacy: The exercise of control New York, NY: W.H. Freeman. 
Bandura, A. (1989). Human agency in social cognitive theory. American psychologist, 44(9), 1175.

Bell, H., Limberg, D., \& Robinson, E. (2013). Recognizing trauma in the classroom: A practical guide for educators. Childhood Education, 89(3), 139-145.

Berghoff, B., Blackwell, S., \& Wisehart, R. (2011). Using Critical Reflection to Improve Urban Teacher Preparation: A Collaborative Inquiry of Three Teacher Educators. Penn GSE Perspectives on Urban Education, 8(2), 19-28.

Bethell, C. D., Newacheck, P., Hawes, E., \& Halfon, N. (2014). Adverse childhood experiences: assessing the impact on health and school engagement and the mitigating role of resilience. Health Affairs, 33(12), 2106-2115.

Bowe, A., Desjardins, C., Covington-Clarkson, L., \& Lawrenz, F. (2017). Urban elementary single-sex math classrooms: Mitigating stereotype threat for African American girls. Urban Education, 52(3), 370-398.

Bride, B., Radey, M., \& Figley, C. (2007). Measuring compassion fatigue. Clinical Social Work Journal, 35(3), 155-163.

Brill, S., \& McCartney, A. (2008). Stopping the revolving door: Increasing teacher retention. Politics \& Policy, 36(5), 750-774.

Brydon-Miller, M. (2012). Addressing the ethical challenges of community-based research. Teaching Ethics, 12(2), 157-162.

Calabrese, R. L., Goodvin, S., \& Niles, R. (2005). Identifying the attitudes and traits of teachers with an at-risk student population in a multi-cultural urban high school. The International Journal of Educational Management, 19(4), 437-449. 
Camangian, P. (2010). Starting with self: Teaching autoethnography to foster critically caring literacies. Research in the Teaching of English, 45(2), 179-204.

Capraro, M. M., Capraro, R. M., \& Helfeldt, J. (2010). Do differing types of field experiences make a difference in teacher candidates' perceived level of competence?. Teacher Education Quarterly, 37(1), 131-154.

Carlson, E. D., Engebretson, J., \& Chamberlain, R. M. (2006). Photovoice as a social process of critical consciousness. Qualitative health research, 16(6), 836-852.

Charner-Laird, M., Szczesiul, S., Kirkpatrick, C. L., Gordon, P., \& Watson, D. (2016). From Collegial Support to Critical Dialogue: Including New Teachers' Voices in Collaborative Work. The Professional Educator, 40(2), 1.

Chio, V. C. M., \& Fandt, P. M. (2007). Photovoice in the diversity classroom: Engagement, voice and the "eye/I" of the camera. Journal of Management Education, 31(4), 484-504.

Clyde, J. A., Barber, S. Z., Hogue, S. L., \& Wasz, L. L. (2006). Breakthrough to meaning: Helping your kids become better readers, writers, and thinkers.

Cochran-Smith, M., Villegas, A. M., Abrams, L., Chavez-Moreno, L., Mills, T., \& Stern, R. (2015). Critiquing teacher preparation research: An overview of the field, part II. Journal of Teacher Education, 66(2), 109-121.

Coffey, H. (2010). “They taught me”: The benefits of early community-based field experiences in teacher education. Teaching and Teacher Education, 26(2), 335342.

Costelloe, S. C. (2006). Characteristics of the 2004-05 cohort of new teachers in Philadelphia. Penn GSE Perspectives on Urban Education, 4(1), 19. 
Creswell, J. W. (2013). Qualitative inquiry and research design. (3rd ed.). Thousand Oaks, CA: SAGE Publications.

Cuthrell, K., Stapleton, J., \& Ledford, C. (2009). Examining the culture of poverty: Promising practices. Preventing School Failure: Alternative Education for Children and Youth, 54(2), 104-110.

Dauksas, L., \& White, J. (2010). Should I stay or should I go? How teacher leadership can improve teacher retention. AASA Journal of Scholarship and Practice, 7(2), 27-32.

Delgado, R. (1996). Rodrigo's eleventh chronicle: Empathy and false empathy. California Law Review, 84(1), 61-100.

Dods, J. (2013). Enhancing understanding of the nature of supportive school-based relationnships for youth who have experienced trauma. Canadian Journal of Education, 36(1), 71-95.

Donaldson, M. L. (2009). Into - and out of — city schools: Retention of teachers prepared for urban settings. Equity \& Excellence in Education, 42(3), 347-370.

Dorman, E. (2012). "everything that's challenging in my school makes me a better teacher": Negotiating tensions in learning to teach for equity. Journal of Urban Learning, Teaching, and Research, 8, 83-92.

Duck, L. (2007). Using sounder foundations to help avoid the" why new teachers cry" phenomenon. The Clearing House: A Journal of Educational Strategies, Issues and Ideas, 81(1), 29-36.

Emdin, C. (2016). For white folks who teach in the hood-- and the rest of y'all too : 
Reality pedagogy and urban education (A simmons college/Beacon press race, education, and democracy series book). Boston, Massachusetts: Beacon Press. Epstein, K. K. (2005). The whitening of the American teaching force: A problem of recruitment or a problem of racism? Social Justice, 32(3), 89-102.

Evans, D. E. (2010). Understanding why principals leave or stay in challenging urban schools (Doctoral dissertation, UC Berkeley).

Fayne, H., \& Matthews, I. (2010). A local solution to a national problem: Preparing preservice candidates for urban middle schools. Professional Educator, 34(1).

Feng, L. (2010). Hire today, gone tomorrow: New teacher classroom assignments and teacher mobility. Education, 5(3), 278-316.

Fetterman, D. (2017). Transformative Empowerment Evaluation and Freirean Pedagogy: Alignment With an Emancipatory Tradition. New Directions for Evaluation, 2017(155), 111-126.

Field, S., \& Gutierrez, C. (2006). Book review: the community teacher: a new framework for effective urban teaching by Peter C. Murrell, Jr. Action in Teacher Education, 27(4), 55-58.

Forster, M., Gower, A. L., McMorris, B. J., \& Borowsky, I. W. (2017). Adverse childhood experiences and school-based victimization and perpetration. Journal of interpersonal violence, 0886260517689885.

Fowler, F. (2013). Policy studies for educational leaders fourth edition: An introduction. Boston: Pearson.

Freedman, S., \& Appleman, D. (2009). "In it for the long haul": How teacher education 
can contribute to teacher retention in high-poverty, urban schools. Journal of Teacher Education, 60(3), 323-337.Gimbert, B., Desai, S., \& Kerka, S. (2010). The big picture: Focusing urban teacher education on the community. Phi Delta Kappan, 92(2), 36-39.

Gless, J., \& Baron, W. (1992). A guide to prepare support providers for work with beginning teachers. Santa Cruz, CA: The California New Teacher Project.

Gomez, M. L. (1993). Prospective teachers' perspectives on teaching diverse children: A review with implications for teacher education and practice. Journal of Negro Education, 63, 459-474.

Gray, L., \& Taie, S. (2015). Public School Teacher Attrition and Mobility in the First Five Years: Results from the First through Fifth Waves of the 2007-08 Beginning Teacher Longitudinal Study. First Look. NCES 2015-337. National Center for Education Statistics.

Green, A. M., Kent, A. M., Lewis, J., Feldman, P., Motley, M. R., Baggett, P. V., Shaw, E. L., Byrd, K., Simpson, J. (2011). Experiences of elementary preservice teachers in an urban summer enrichment program. Western Journal of Black Studies, 35(4), 227-239.

Haberman, M. (1987). Recruiting and Selecting Teachers for Urban Schools. ERIC/CUE Urban Diversity Series, Number 95.

Haberman, M. (2005). Selecting and preparing urban teachers. Retrieved March, 9, 2010. Hammerness, K., \& Matsko, K. K. (2013). When context has content: A case study of new teacher induction in the university of Chicago's urban teacher education program. Urban Education, 48(4), 557-584. 
Harris, N. B. (2015). How childhood trauma affects health across a lifetime. Retrieved From https://www.ted.com/talks/nadine_burke_harris_how_childhood_trauma_affects health_across_a_lifetime?language=en

Hein, S. F., \& Austin, W. J. (2001). Empirical and hermeneutic approaches to phenomenological research in psychology: A comparison. Psychological methods, 6(1), 3 .

Helfeldt, J. P., Capraro, R. M., Capraro, M. M., Foster, E., \& Carter, N. (2009). An urban schools-university partnership that prepares and retains quality teachers for "high need" schools. Teacher Educator, 44(1), 1-20

Hoban, G. F. (2005). The missing links in teacher education design: Developing a multilinked conceptual framework (Self study of teaching and teacher education practices; Self study of teaching and teacher education practices). Dordrecht: Springer.

Hunt, T. K., Slack, K. S., \& Berger, L. M. (2017). Adverse childhood experiences and behavioral problems in middle childhood. Child abuse \& neglect, 67, 391-402.

Ingersoll, R., Merrill, L., \& Stuckey, D. (2014). Seven trends: The transformation of the teaching force.

Ingersoll, R. M., \& Smith, T. M. (2003). The wrong solution to the teacher shortage. Educational leadership, 60(8), 30-33.

J. Colley (personal communication, April 11, 2017)

Jacob, B. (2007). The challenges of staffing urban schools with effective teachers. Future of Children, 17(1), 129-153. 
Janesick, V. (2013). Oral history, life history, and biography. In A. Trainor \& E. Graue (Eds.), Reviewing qualitative research in the social sciences (pp. 180-196). New York: Routledge.

Jensen, E. (2009). Teaching with poverty in mind. Alexandria, VA: ASCD.

Johnson Iii, J. A., \& Johnson, A. M. (2015). Urban-rural differences in childhood and adolescent obesity in the United States: a systematic review and meta-analysis. Childhood Obesity, 11(3), 233-241.

Kaiser, A. (2011). Beginning Teacher Attrition and Mobility: Results from the First through Third Waves of the 2007-08 Beginning Teacher Longitudinal Study. First Look. NCES 2011-318. National Center for Education Statistics.

Kentucky department of education: Professional growth and effectiveness system. Retrieved from http://education.ky.gov/TEACHERS/PGES/Pages/PGES.aspx

Krueger, R. A., \& Casey, M. A. (2002). Designing and conducting focus group interviews.

Kukla-Acevedo, S. (2009). Leavers, movers, and stayers: The role of workplace conditions in teacher mobility decisions. The Journal of Educational Research, $102(6), 443-452$.

Ladson-Billings, G. J. (1999). Chapter 7: Preparing teachers for diverse student populations: A critical race theory perspective. Review of Research in Education, 24(1), 211-247.

Ledoux, K. (2015). Understanding compassion fatigue: Understanding compassion. Journal of Advanced Nursing, 71(9), 2041-2050. doi:10.1111/jan.12686

Maclean, K., \& Woodward, E. (2013). Photovoice Evaluated: An Appropriate Visual 
Methodology for A boriginal Water Resource Research. Geographical Research, 51(1), 94-105.

Martin, J. (1991). The trauma of homelessness. International Journal of Mental Health, 20(2), 17-27.

Matus, D. E. (1999). An innovative strategy supports student teachers in urban secondary schools. The Clearing House, 73(1), 37-41.

McKinney, S., Haberman, M., Stafford-Johnson, D., \& Robinson, J. (2008). Developing Teachers for High-Poverty Schools. Urban Education, 43(1), 68-82.

Metzler, M., Merrick, M. T., Klevens, J., Ports, K. A., \& Ford, D. C. (2017). Adverse childhood experiences and life opportunities: shifting the narrative. Children and youth services review, 72, 141-149.

Miles, M., Huberman, A., \& Saldaña, J. (2014). Qualitative data analysis: A methods sourcebook ( $3^{\text {rd }}$ ed.). Thousand Oaks, CA: Sage Publishers.

Milner IV, H. R. (2007). Race, culture, and researcher positionality: Working through dangers seen, unseen, and unforeseen. Educational researcher, 36(7), 388-400.

Milner, R. H., \& Laughter, J. C. (2015). But good intentions are not enough: Preparing teachers to center race and poverty. Urban Review: Issues and Ideas in Public Education, 47(2), 341-363.

Mohammed, S. \& Sajun, S., Photovoice manual. Retrieved from http://www.irdresearch.org/wp-content/uploads/2015/03/Photovoice-Manual$\underline{\text { Revised.pdf }}$

Moje, E. B., Ciechanowski, K. M., Kramer, K., Ellis, L., Carrillo, R., \& Collazo, T. 
(2004). Working toward third space in content area literacy: An examination of everyday funds of knowledge and discourse. Reading research quarterly, 39(1), 38-70.

Moll, L. C., Amanti, C., Neff, D., \& Gonzalez, N. (1992). Funds of knowledge for teaching: Using a qualitative approach to connect homes and classrooms. Theory into practice, 31(2), 132-141.

Morgan, M. M., \& Kritsonis, W. A. (2008). A national focus: The recruitment, retention, and development of quality teachers in hard-to-staff schools. National Journal for Publishing and Mentoring Doctoral Student Research, 5(1), 1-7.

Murrell, P. (2000). Community teachers: a conceptual framework for preparing exemplary urban teachers. The Journal of Negro Education, 69(4), 338-348.

Murrell, P. (2001). The community teacher : A new framework for effective urban teaching. New York: Teachers College Press.

Nahal, S. P. (2010). Voices from the field: Perspectives of first-year teachers on the disconnect between teacher preparation programs and the realities of the classroom. Research in Higher Education Journal, 8, 1-19.

Noel, J. (2010). Weaving teacher education into the fabric of urban schools and communities. Teacher Education Quarterly, 37(3), 9-25.

Oakes, J., Franke, M., Quartz, K., \& Rogers, J. (2002). Research for high-quality urban teaching: Defining it, developing it, assessing it. Journal of Teacher Education, 53(3), 228-34.

Olsen, B., \& Anderson, L. (2007). Courses of action: A qualitative investigation into urban teacher retention and career development. Urban Education, 42(1), 5-29. 
Onore, C., \& Gildin, B. (2010). Preparing urban teachers as public professionals through a university-community partnership. Teacher Education Quarterly, 37(3), 27-44.

Ostovar-Nameghi, S. A., \& Sheikhahmadi, M. (2016). From teacher isolation to teacher collaboration: Theoretical perspectives and empirical findings. English Language Teaching, 9(5), 197.

Paliboda B., Krieg, B., Murdock, L., \& Havelock, J., A practical guide to photovoice: Sharing pictures, telling stories and changing communities. Retrieved from http://www.pwhce.ca/photovoice/pdf/Photovoice_Manual.pdf

Papay, J., \& Johnson, S. (2012). Is par a good investment? Understanding the costs and benefits of teacher peer assistance and review programs. Educational Policy, 26(5), 696-729.

Porter, E, \& Cohen, M. (2013). Phenomenology. In A. Trainor \& E. Graue (Eds.), Reviewing qualitative research in the social sciences (pp. 180-196). New York: Routledge.

Prilleltensky, I., Neff, M., \& Bessell, A. (2016). Teacher stress: what it is, why it's important, how it can be alleviated. Theory Into Practice, 55(2), 104-111.

Prince, C. D. The Challenge of Attracting Good Teachers and Principals to Struggling Schools. American Association of School Administrators, "Issues and Insights.”(Arlington, VA, January 2002).

Proctor, T., Rentz, N., \& Jackson, M. (2001). Preparing teachers for urban schools: The role of field experiences. Western Journal of Black Studies, 25(4), 219-27.

Ryan, A., Ensminger, D., Heineke, A., Kennedy, A., Prasse, D., \& Smetana, L. (2014). 
Teaching, learning, and leading with schools and communities: One urban university re-envisions teacher preparation for the next generation. Issues in Teacher Education, 22(2), 139-153.

Saldaña, J. (2016). The Coding Manual for Qualitative Researchers. ( $3^{\text {rd }}$ ed.). Thousand Oaks, CA: Sage.

Schultz, K., Jones-Walker, C. E., \& Chikkatur, A. P. (2015). Listening to students, negotiating beliefs: Preparing teachers for urban classrooms. Curriculum Inquiry, $38(2), 155-187$.

Schunk, D. H., \& DiBenedetto, M. K. (2016). Self-efficacy theory in education. Handbook of motivation at school, 2, 34-54.

Sharp Donahoo LM, Siegrist B, and Garrett-Wright D. 2017. “Addressing Compassion Fatigue and Stress of Special Education Teachers and Professional Staff Using Mindfulness and Prayer." The Journal of School Nursing : The Official Publication of the National Association of School Nurses 1059840517725789:1059840517725789-89.

Showalter, S. (2010). Compassion fatigue: What is it? why does it matter? recognizing the symptoms, acknowledging the impact, developing the tools to prevent compassion fatigue, and strengthen the professional already suffering from the effects. American Journal of Hospice and Palliative Medicine, 27(4), 239-242.

Simon, F. (2011). Efficacy development in new teacher study groups (Doctoral dissertation, 2011). UNIVERSITY OF ARIZONA.

Simon, N. S., \& Johnson, S. M. (2015). Teacher turnover in high-poverty schools: What we know and can do. Teachers College Record, 117(3), 1-36. 
Sitler, H. C. (2009). Teaching with awareness: The hidden effects of trauma on learning. The Clearing House: A Journal of Educational Strategies, Issues and Ideas, 82(3), 119-124.

Smith, T. M., \& Ingersoll, R. M. (2004). What are the effects of induction and mentoring on beginning teacher turnover?. American educational research journal, 41(3), 681-714.

Snoeck, I., \& Struyf, E. (2012). The inservice-teacher-training in Flemish schools: Does practice make a (more) perfect teacher? Journal of Workplace Learning, 24(4), 286-298.

Stolbach, B. C., Minshew, R., Rompala, V., Dominguez, R. Z., Gazibara, T., \& Finke, R. (2013). Complex trauma exposure and symptoms in urban traumatized children: A preliminary test of proposed criteria for developmental trauma disorder. Journal of Traumatic Stress, 26(4), 483-491.

Strawn, C. A., Fox, R. K., \& Duck, L. (2008). Preventing teacher failure: Six keys to success in moving beyond the "sink or swim" mentality. The Clearing House, 81(6), 271-277.

Strier, R., \& Shechter, D. (2016). Visualizing access: knowledge development in university-community partnerships. Higher Education, 71(3), 343-359.

Taylor, A., \& Frankenberg, E. (2009). Exploring urban commitment of graduates from urban-focused teacher education program. Equity \& Excellence in Education, 42(3), 327-346.

Ullucci, K., \& Howard, T. (2015). Pathologizing the poor: Implications for preparing teachers to work in high-poverty schools. Urban Education, 50(2), 170-193. 
United States. (2015). Every student succeeds act. Washington, D.C.: U.S. Government Publishing Office. (2015).

United States. Department of Education. Policy and Program Studies Service. (2007).

State and local implementation of the no child left behind act. Washington, D.C.:

U.S. Department of Education, Office of Planning, Evaluation and Policy

Development, Policy and Program Studies Service. (2007).

Valenzuela, A. (1999). Subtractive schooling: U.S.-Mexican youth and the politics of

caring (SUNY series, the social context of education; SUNY series, social context of education). Albany: State University of New York Press.

Valenzuela, A., \& Rubio, B. (2018). Subtractive schooling. The TESOL Encyclopedia of English Language Teaching, 1-7.

Van Manen, M. (1984). "Doing" phenomenological research and writing: An introduction (department of secondary education, faculty of education). Alberta: University of Alberta.

Van Manen, M. (2007). Phenomenology of practice. Phenomenology \& Practice, 1(1), 11-30.

Velliaris, D. M. (2016). Culturally Responsive Pathway Pedagogues: Respecting the Intricacies of Student. Handbook of research on effective communication in culturally diverse classrooms, 18-34.

Waddell, J. H. (2010). Fostering relationships to increase teacher retention in urban schools. Journal of Curriculum and Instruction, 4(1), 70-85.

Wade, R., Shea, J. A., Rubin, D., \& Wood, J. (2014). Adverse childhood experiences of low-income urban youth. Pediatrics, peds-2013. 
Wang, C. C., Morrel-Samuels, S., Hutchison, P. M., Bell, L., \& Pestronk, R. M. (2004). Flint photovoice: Community building among youths, adults, and policymakers. American journal of public health, 94(6), 911-913.

Wang, C., \& Burris, M. A. (1997). Photovoice: Concept, methodology, and use for participatory needs assessment. Health education \& behavior, 24(3), 369-387.

Warren, C. A., \& Hotchkins, B. K. (2015). Teacher education and the enduring significance of "false empathy". The Urban Review, 47(2), 266-292.

Waxman, H. C., \& Huang, S. L. (1996). Motivation and learning environment differences in inner-city middle school students. Journal of Educational Research, 90(2), 93102.

Yosso, T. J. (2005). Whose culture has capital? A critical race theory discussion of community cultural wealth. Race Ethnicity and Education, 8(1), 69-91.

Zenkov, K., \& Harmon, J. (2009). Picturing a writing process: Photovoice and teaching writing to urban youth. Journal of Adolescent \& Adult Literacy, 52(7), 575-584 
APPENDIX A

Study Participant Snapshots

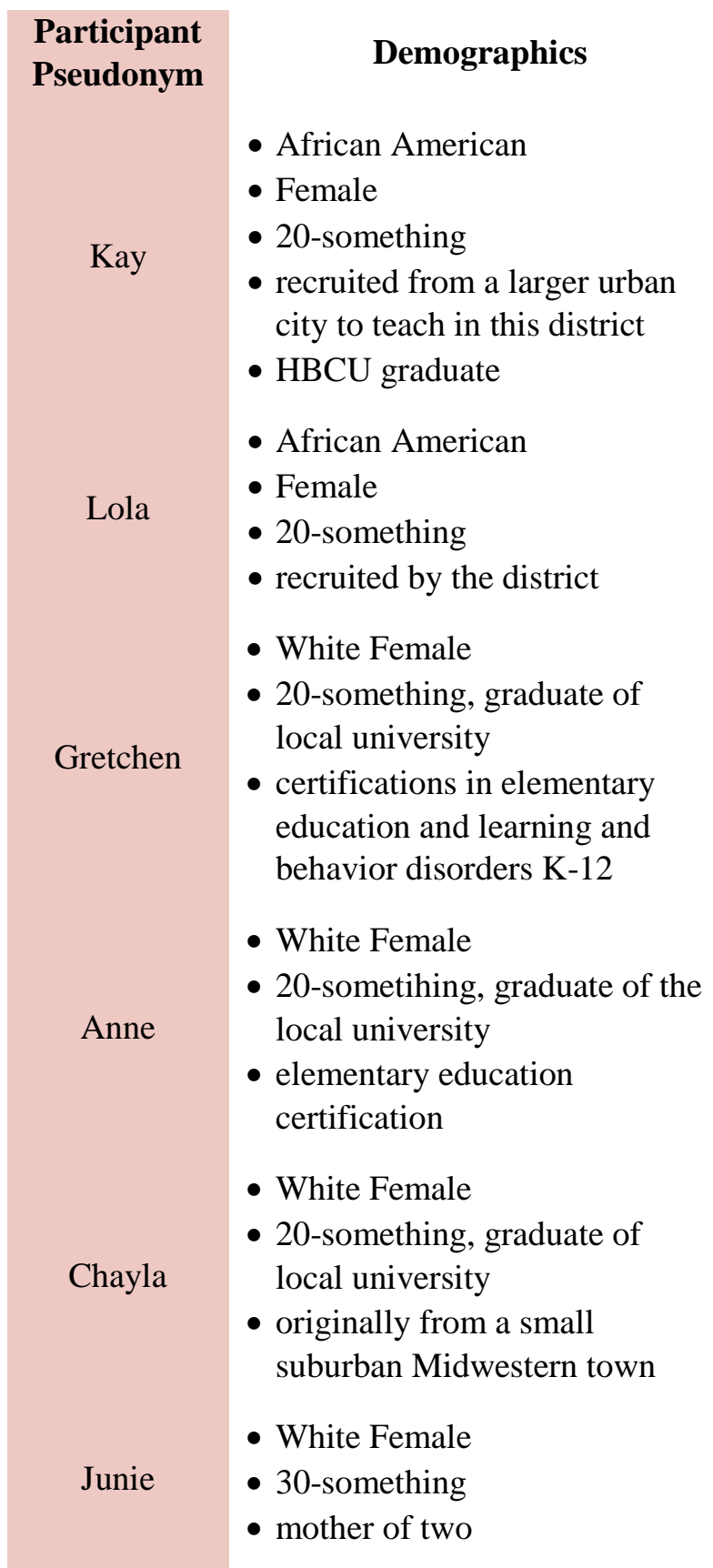
First grade $\quad$ Outspoken
teacher in - Frustrated
school A

First grade $\quad$ calm

teacher in $\bullet$ frustrated

school A

First grade $\quad$ free-spirited

teacher in $\bullet$ independent thinker

school B

- reflective
Fifth grade $\bullet$ energetic

teacher in $\quad$ pro black lives matter

school B • determined

Second grade teacher in

school C
- quiet

- frustrated

- disappointed 
- new to area from a small Midwestern town

- wife of seminary student

- middle science and language arts endorsements 
APPENDIX B

Demographics of Focus District and Schools

\begin{tabular}{|c|c|c|c|c|c|c|c|c|}
\hline Location & $\begin{array}{c}\text { Teacher } \\
\text { Participants }\end{array}$ & $\begin{array}{c}\text { 4-year } \\
\text { Average } \\
\text { Teacher } \\
\text { Retention } \\
\text { Rate }\end{array}$ & $\begin{array}{l}\text { Retention } \\
\text { Rate } \\
\text { Ranking } \\
\text { Among the } \\
91 \text { District } \\
\text { Schools }\end{array}$ & $\begin{array}{l}\text { African- } \\
\text { American } \\
\text { Students }\end{array}$ & $\begin{array}{c}\text { White } \\
\text { American } \\
\text { Students }\end{array}$ & $\begin{array}{l}\text { Hispanic } \\
\text { Students }\end{array}$ & $\begin{array}{c}\text { Other } \\
\text { Students }\end{array}$ & $\begin{array}{c}\text { Total } \\
\text { Number of } \\
\text { Students }\end{array}$ \\
\hline District & 6 & $88.8 \%$ & -- & $35 \%$ & $42 \%$ & $12 \%$ & $11 \%$ & $\begin{array}{c}>43,700 \\
\text { elementary } \\
\text { level } \\
\text { students }\end{array}$ \\
\hline $\begin{array}{c}\text { Focus } \\
\text { School A }\end{array}$ & $\begin{array}{l}2 \text { African- } \\
\text { American } \\
\text { Females }\end{array}$ & $86.5 \%$ & $\begin{array}{l}67^{\text {th }} \text { of } 91 \\
\text { schools }\end{array}$ & $69 \%$ & $13 \%$ & $8 \%$ & $9 \%$ & 370 \\
\hline $\begin{array}{c}\text { Focus } \\
\text { School B }\end{array}$ & $\begin{array}{l}3 \text { White } \\
\text { American } \\
\text { Females }\end{array}$ & $82.7 \%$ & $\begin{array}{c}82^{\text {nd }} \text { of } 91 \\
\text { schools }\end{array}$ & $79 \%$ & $10 \%$ & $7 \%$ & $4 \%$ & 578 \\
\hline $\begin{array}{c}\text { Focus } \\
\text { School C }\end{array}$ & $\begin{array}{l}1 \text { White } \\
\text { American } \\
\text { Female }\end{array}$ & $76.6 \%$ & $\begin{array}{c}89^{\text {th }} \text { of } 91 \\
\text { schools }\end{array}$ & $62 \%$ & $14 \%$ & $15 \%$ & $9 \%$ & 393 \\
\hline
\end{tabular}


APPENDIX C

Data Collection Schedule

$\begin{array}{llll}\text { Event } & \text { Frequency } & \text { Focus } & \text { Date } \\ \begin{array}{l}\text { Researcher } \\ \text { Reflection Journal }\end{array} & \begin{array}{l}\text { Weekly in } \\ \text { the field }\end{array} & \begin{array}{l}\text { Place holding noticings, } \\ \text { patterns, etc. }\end{array} & \text { Weekly } \\ \begin{array}{l}\text { Participant } \\ \text { Reflection Journals } \\ \text { PhotoVoice }\end{array} & \begin{array}{l}\text { Three } \\ \text { Events }\end{array} & \begin{array}{l}\text { Impact of experiences on } \\ \text { perceptions }\end{array} & \text { Weekly } \\ \text { One Event } & \begin{array}{l}\text { Visual evidence of lived } \\ \text { experience }\end{array} & \text { November 20, 2017 } \\ \text { Focus Group } & \text { Two Events } & \begin{array}{l}\text { Gather reflective thoughts } \\ \text { on lived experiences }\end{array} & \text { November 11, 2017 }\end{array}$

December 9, 2017 


\section{APPENDIX D}

\section{Urban Competencies Professional Development Plan}

Collaborative induction experience with newly hired JCPS teachers as part of the research study

\begin{tabular}{|c|c|c|c|}
\hline Date/Time & $\begin{array}{c}\text { PD } \\
\text { Hours }\end{array}$ & $\begin{array}{c}\text { Focus/Urban } \\
\text { Competency Pillars }\end{array}$ & Facilitated by \\
\hline November 11,2017 & 2 hours & $\begin{array}{l}\text { Cultural Competence } \\
\text { in Urban settings }\end{array}$ & $\begin{array}{l}\text { Nyree Clayton-Taylor, } 2018 \text { Kentucky } \\
\text { Elementary Teacher of the Year, Hip-Hop } \\
\text { Based Curriculum Developer, and Writing } \\
\text { Resource Teacher }\end{array}$ \\
\hline November 11,2017 & 2 hours & $\begin{array}{l}\text { Community-Based } \\
\text { Teaching in Urban } \\
\text { Settings }\end{array}$ & $\begin{array}{l}\text { Missy Callaway, Veteran High School Teacher, } \\
\text { Doctoral Candidate, and Goal Clarity Coach }\end{array}$ \\
\hline November 11,2017 & 2 hours & $\begin{array}{l}\text { Poverty-Informed } \\
\text { Practices in Urban } \\
\text { Settings }\end{array}$ & $\begin{array}{l}\text { Dr. Larhondalyn Mathies, High School Goal } \\
\text { Clarity Coach }\end{array}$ \\
\hline November 15, 2017 & 2 hours & $\begin{array}{l}\text { Cultural Competence } \\
\text { in Urban settings }\end{array}$ & $\begin{array}{l}\text { Sandra Hogue, Veteran Elementary Educator, } \\
\text { Doctoral Candidate, Goal Clarity Coach }\end{array}$ \\
\hline November 20, 2017 & 2 hours & $\begin{array}{l}\text { Community- } \\
\text { Informed Practices } \\
\text { in Urban Settings }\end{array}$ & $\begin{array}{l}\text { Harvetta Broadway-Ray, Adjunct Professor in } \\
\text { Department of Social Work at local university } \\
\text { and Certified Social Worker servicing local } \\
\text { community non-profit organization }\end{array}$ \\
\hline November 29, 2017 & 1 hour & $\begin{array}{l}\text { Poverty-Informed } \\
\text { Practices in Urban } \\
\text { Settings }\end{array}$ & $\begin{array}{l}\text { Shelli Barber, Veteran Urban Elementary } \\
\text { Educator and Author }\end{array}$ \\
\hline November 29, 2017 & 1 hour & $\begin{array}{l}\text { PhotoVoice } \\
\text { Experience }\end{array}$ & Teacher Participants \\
\hline
\end{tabular}




\begin{tabular}{|l|l|l|l|}
\hline December 9, 2017 & $\begin{array}{l}2.5 \\
\text { hours }\end{array}$ & $\begin{array}{l}\text { Cultural Competence } \\
\text { in Urban Settings }\end{array}$ & $\begin{array}{l}\text { Dr. Detra Johnson, Assistant Professor in } \\
\text { Educational Leadership, Evaluation, and } \\
\text { Organizational Development }\end{array}$ \\
\hline December 9, 2017 & $\begin{array}{l}2.5 \\
\text { hours }\end{array}$ & $\begin{array}{l}\text { Poverty-Informed } \\
\text { Practices in Urban } \\
\text { Settings }\end{array}$ & $\begin{array}{l}\text { Dr. Jean Anne Clyde, Educational Consultant, } \\
\text { Former Professor Teacher Preparation Program }\end{array}$ \\
\hline
\end{tabular}




\section{APPENDIX E \\ Focus Group Protocol}

Set up and test recording devices.

$>$ Thank participants for being part of the study.

$>$ Remind participants of the focus of the study: urban teacher readiness and competencies

Remind participants that the session is being recorded and that pseudo names should be used.

$>$ Inform participants that you and/or others may ask probing or clarifying questions as part of the conversation.

Invite participants to ask questions.

The following demographic questions will be asked only at the beginning of the first focus group event.

1. What is your age?

2. What certifications do you hold?

3. In what part of the city do you live?

4. Was your K-12 education public, private or other?

\section{$\underline{\text { Part A - Urban }}$}

5. How would you define the word urban?

6. What are the greatest challenges facing urban education?

7. What are the greatest benefits of urban education?

8. What skills, strategies or assets do competent urban educators possess?

\section{Part B - Poverty}

9. What is the primary cause of poverty in urban settings?

10. In what ways does poverty manifest itself in classrooms?

$$
\underline{\text { Part C-Cultural Competence }}
$$

11. How does culture impact the classroom?

12. How would you describe cultural competence?

$$
\text { Part D - Urban Community }
$$

13. What resources are available to support the prosperity of the urban community?

14. How does the urban community impact what happens in urban classroom? 


\section{Part E - Self Assessment}

15. Are you an urban-ready teacher? If so, what makes you ready? If not, why do you feel you are not?

16. How should new urban teachers be made urban-ready? 


\section{APPENDIX F \\ Participant Journal Writing Guide Protocol \\ (Adapted from the work of McCauley-Kate, 2003)}

Each research participant will be writing a weekly journal entry over the course of phase two of the research study. The goal of these journal entries is to capture the essence of your lived experiences as you navigate through and make sense of your work in this induction program.

Part A - Describing a significant event

1. Take time to reflect on one event that was of importance to you this week. The event was significant to you because it bothered, excited, and/or caused you to reflect or rethink your initial ideas about teaching in general and/or teaching in an urban setting. Ultimately, the event chosen should be one that spurred reflection and one that you learned from.

2. Describe the event in specific detail. Recreate details of the experience including what was said and done, and how things looked. Describe the circumstances that brought on the event/s, what was happening at the time. 
NOTE: Try to avoid making inferences about how people felt or what they thought. Your feelings should be included in Part B, the analysis of the event.

$$
\text { Part B - Analysis of Event }
$$

1. Discuss the feelings and thoughts you believe may have caused the event to occur.

2. Explain why you believe those feelings/thoughts were significant.

3. What were/are you wondering about this event?

4. What did you learn from the event or what new thoughts are you thinking about the event?

5. Identify any challenges that have emerged from the event and how you plan to or did follow up.

6. Did the event confirm your perspective or ideas of teaching in an urban setting or challenge you to reconsider those perspectives or ideas? 


\section{APPENDIX G}

\section{PhotoVoice Experience Protocol}

(Adapted from the work of Mohammed, S. \& Sajun, S.and the work of Paliboda B.,

$$
\text { Krieg, B., Murdock, L., \& Havelock, J) }
$$

1. Participants will be encouraged to use cameras (camera phones) to capture two photos that reflect the essence of their lived experiences as participants in this induction experience.

2. Select the two most compelling images to share in a PhotoVoice participatory group analysis. Images will be emailed or texted to Sandra Hogue at

\section{Sandra.hogue@ and (502)}

3. Images will be printed on $8 \times 10$ photo paper.

4. Participants will prepare a museum-like artist's title and descriptive statement to accompany each image.

5. I will use all images and artist statements to create a gallery for the focus group discussion.

6. Participants will view each image and accompanying artist's statement while engaged in group discourse

7. Each participant will be invited to add their own title and statement one image that resonates with them most. 
8. This gallery will support a discussion regarding the essence of the experiences using these questions:

a. Do you see any similarities in the kind of topic/issue these photographs represent?

b. Do some of these photographs address the same theme? Which ones?

c. What kinds of themes are emerging?

d. What do these images say about urban education?

9. The group of artist-participants will arrange the images in the gallery in ways that are personally and/or collectively meaningful to them for additional discuss.

What do the images you have shared and see in this gallery say about your lived experiences as newly hired urban educators in this collaborative induction program? 


\section{APPENDIX $\mathrm{H}$}

\section{PhotoVoice Gallery of Images, Placards, and Participant Feedback}

\section{Image \\ Number}

1

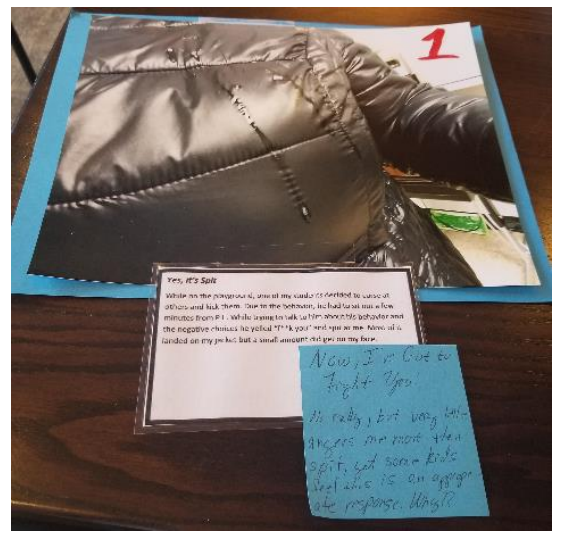

2

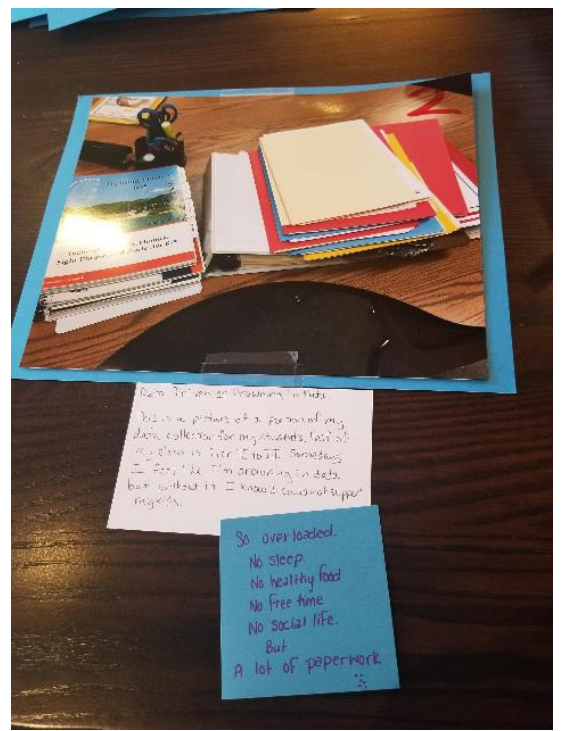

Contributing

Artist/

Participant

Artist's Statement

Yes, it's Spit

While on the playground, one of my students decided to curse at other and kick them. Due to the behavior, he had to sit out a few minutes from P.E. While trying to talk to him about his behavior and the negative choices, he yelled, "f**k you" and spit at me. Most of it landed on my jacket, but a small amount did get on my face.

Data-Driven or Drowning in Data

This is a picture of a portion of my data collection for my students. Half of my class is Tier II to III. Some days I feel like I'm drowning in data but Junie without it, I know I could not support my kids.

\section{Feedback from \\ PhotoVoice \\ Discussion}

Now, I've Got to

Fight You!

Not really, but very little angers me more than spit, yet some kids feel this is an appropriate response. Why??

So overloaded.

No sleep.

No healthy food

No free time

No social life.

But

A lot of paperwork. 


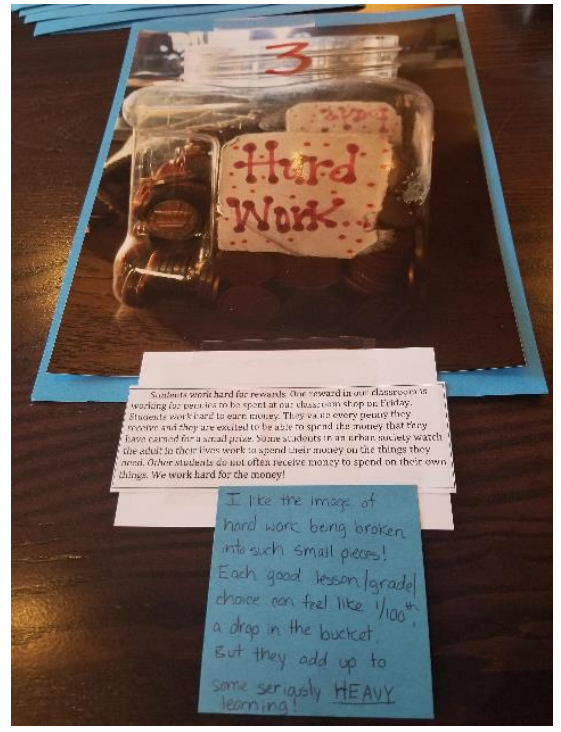

4

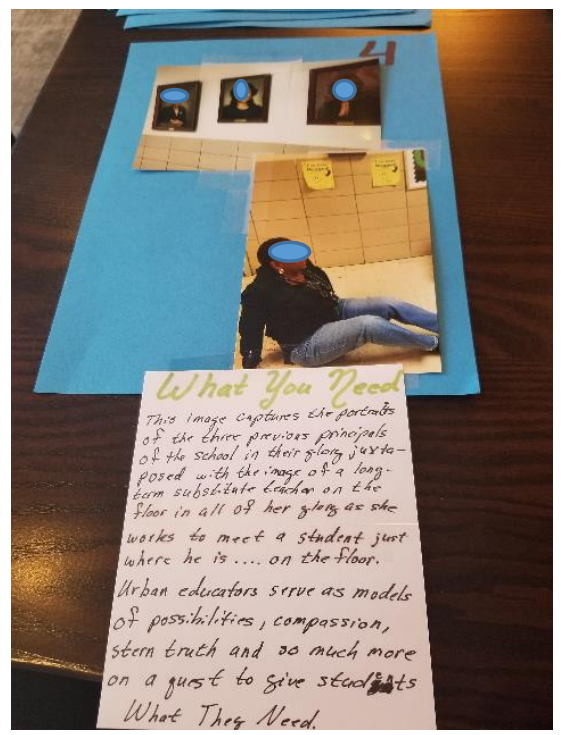

5

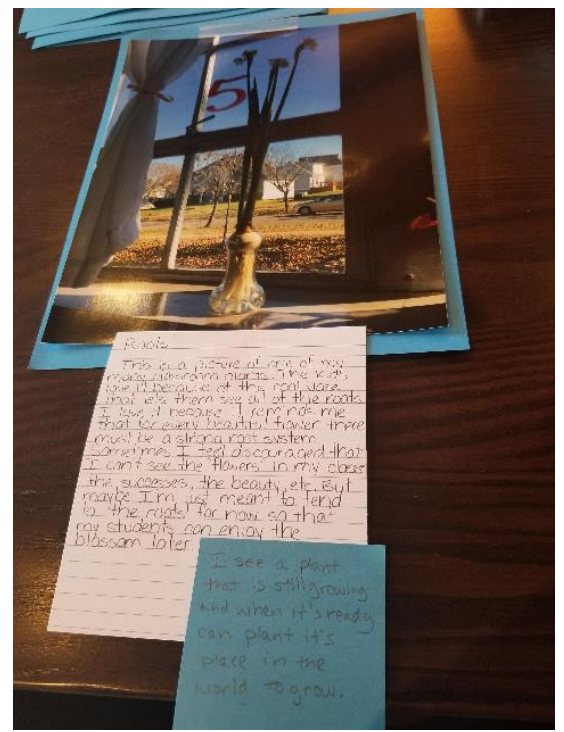

Students work hard for rewards. One reward in our classroom is working for pennies to be spent at our classroom shop on Friday. Students work hard to earn money. They value every penny they receive and they are excited to be able to spend the money that they have earned for a small prize. Some students in an urban society watch the adult in their lives work to spend their money on the things they need. Oher students do not often receive money to spend on their own things. We work hard for the money!

\section{What You Need}

This image captures the portraits of the three previous principals of the school in their glory, juxtaposed with the image of a long-term substitute teacher on the floor in all of her glory as she works to meet a student just where he is...on the floor. Urban educators serve a models of possibilities, compassion, stern truth, and so much more on a quest to give students What They Need

\section{Roots}

This is a picture of one of my many classroom plants. The kids love it because of the cool vase that lets them see all of the roots. I love it because it reminds me that for every beautiful flower,

Gretchen
I like the image of hard work being broken into such small pieces! Each good

lesson/grade/choice can feel like $1 / 100^{\text {th }}$ a drop in the bucket. But they add up to some seriously HEAVY learning!
I see a plant that is still growing, and when it's ready, can plant its place in the world to grow. 

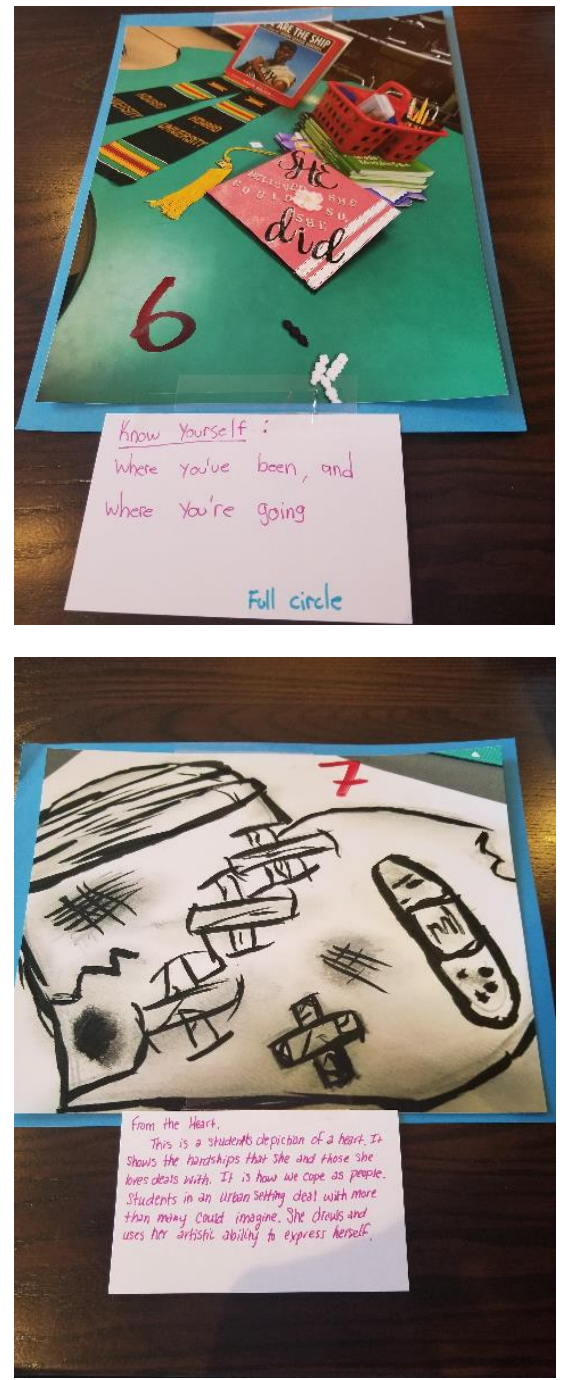

8

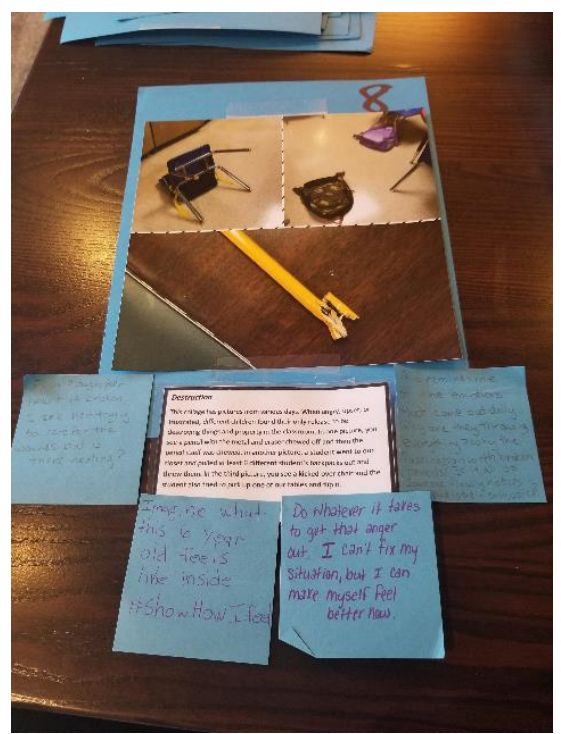

\section{Know Yourself!}

Where you've been, and where you're going
Kay

Chayla

Lola

\section{From the Heart}

This is a students' depiction of a heart. It shows the hardships that she and those she loves deals with. It is how we cope as people. Students in an urban setting deal with more than many could imagine. She draws and uses her artistic ability to express herself.

\section{Destruction}

This collage has pictures from various days. When angry, upset, or frustrated, different children found their only release to be destroying things and property in the classroom. In one picture, you see a pencil with the metal and eraser chewed off and then the pencil itself was chewed. In another picture, a student went to our closet and pulled at least six different students' backpacks out and threw them. In the third picture, you see a kicked over chair and the student also tried to pick up one of our tables and flip it.

\section{Full circle}

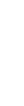


9

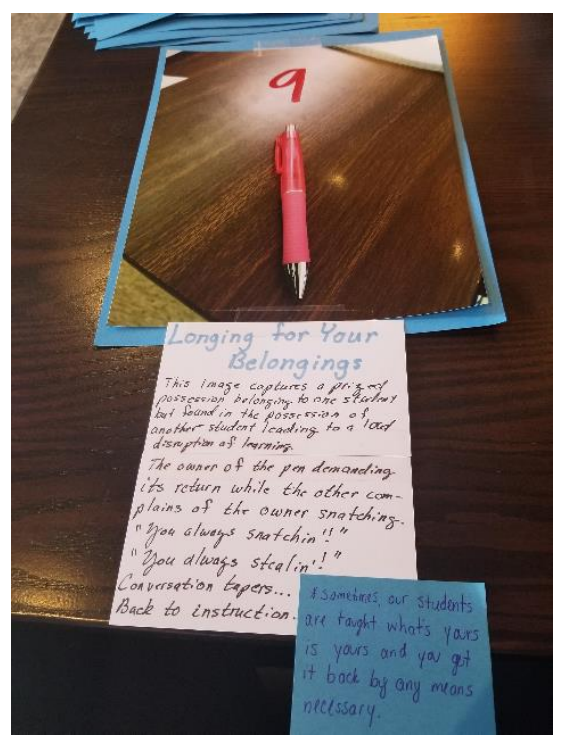

10

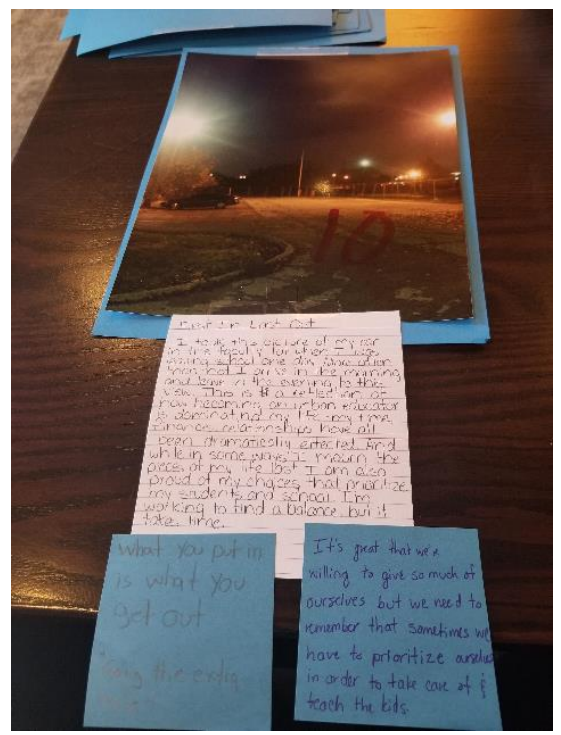

\section{Longing for Your} Belongings

This image captures a prized possession belonging to one student but found in the possession of another student leading to a loud disruption of learning.

Sandra

\section{The owner of the pen} demanding its return while the other complains of the owner snatching.

"You always snatchin'!"

"You always stealin'!"

Conversation tapers ...

Back to instruction...

\section{First In, Last Out}

I took this picture of my car in the faculty lot when I was leaving school on day. More often than not, I arrive in the morning and leave in the evening to this view. This is a reflection of how becoming an urban

Gretchen educator is dominating my life - my time, finances, relationships have all been dramatically affected. And while in some ways I mourn the pieces of my life lost, I am also proud of my choices that prioritize my students and school. I'm working to find a balance, but it takes time. broken pencils? Is it all so someone finally notices them, And is this their only voice?

Sometimes our student are taught what's yours is yours and you get it back by any means necessary.
It's great that we're willing to give so much of ourselves, but we need to remember that sometimes we have to prioritize ourselves in order to take care of and teach the kids.

What you put in is what you get out. "Going the extra mile" 


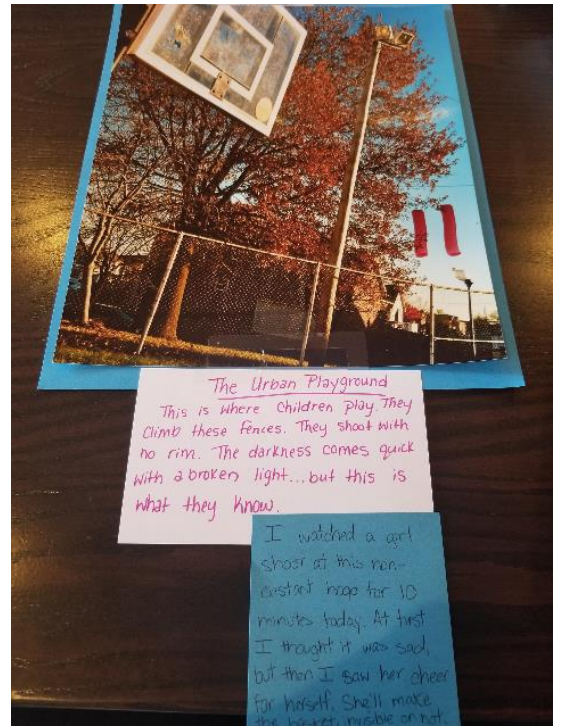

12

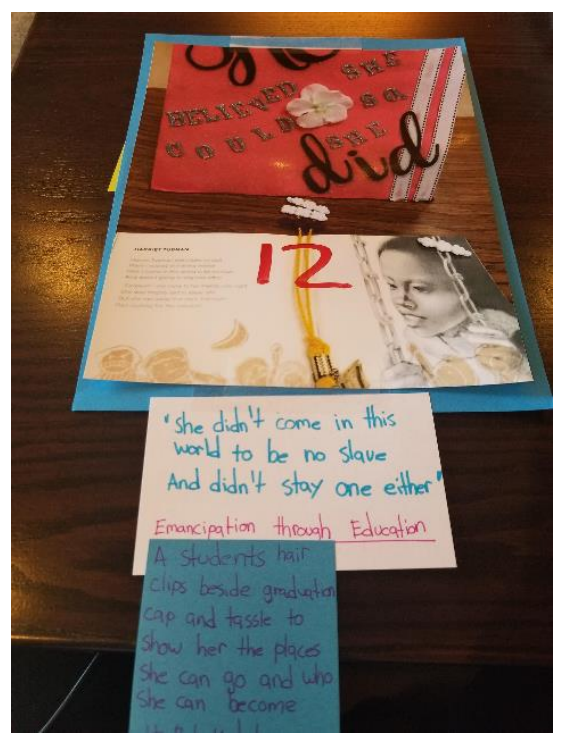

13

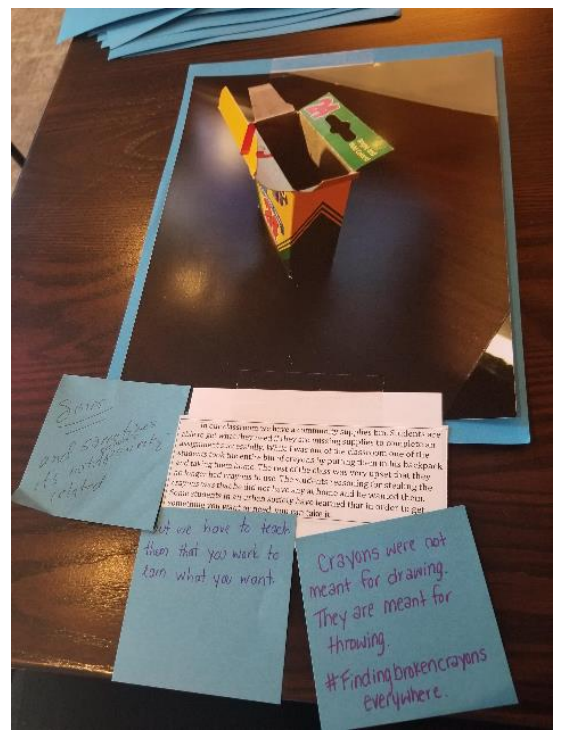

\section{The Urban Playground}

This is where children play. They climb these fences. They shoot with no rim. The darkness comes quick with a broken light ... but this is what they know.

Chayla

Kay

Ann
"She didn't come in this world to be no slave either"
And didn't stay one
I watched a girl shoot at this non-existent hoop for 10 minutes today. At first I thought it was sad, but then I saw her cheer herself. She'll make the basket, invisible or not.
Emancipation through Education A students hair clips beside graduation cap and tassel to show her the places she can go and who she can become \#RoleModel
In our classroom we have community supply bins. Students are able to get what they need if they are missing supplies to complete an assignment successfully. While I was out of the classroom, one of the students took the entire bin of crayons by putting them in his backpack and taking them home. The rest of the class was very upset that they no longer had crayons to use. The student's reasoning for stealing he crayons was that he did not have any at home and wanted them. Some students in an urban society have learned that in order to
But you have to teach them that you work to earn what you want.

Crayons were not meant for drawing. They are meant for throwing. \#FindingBrokenCray onsEverywhere

Some and sometimes it's not economic poverty-related 
14

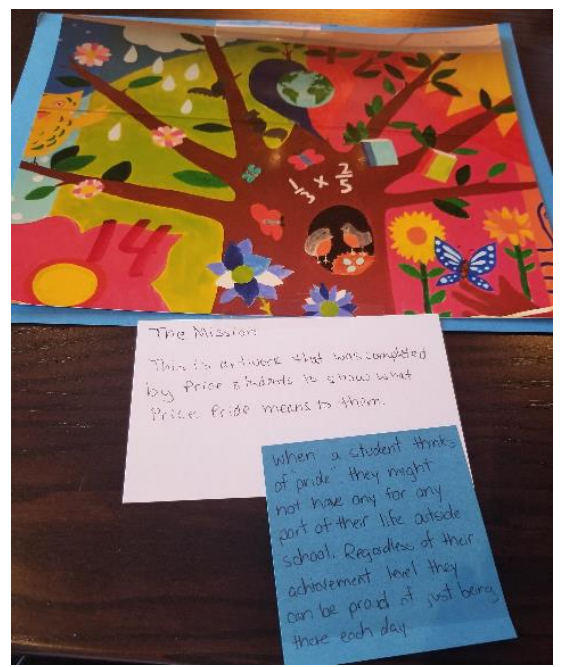

get something you want or need, you can take it.

The Mission

This is artwork that was completed by (School Name) students to show what (School Name)

Pride means to them.

Junie
When a student thinks of pride, they might not have any for any part of their life outside school. Regardless of their achievement level, they can be proud of just being there each day. 
APPENDIX I

Lived Experiences of First Year Urban Teachers

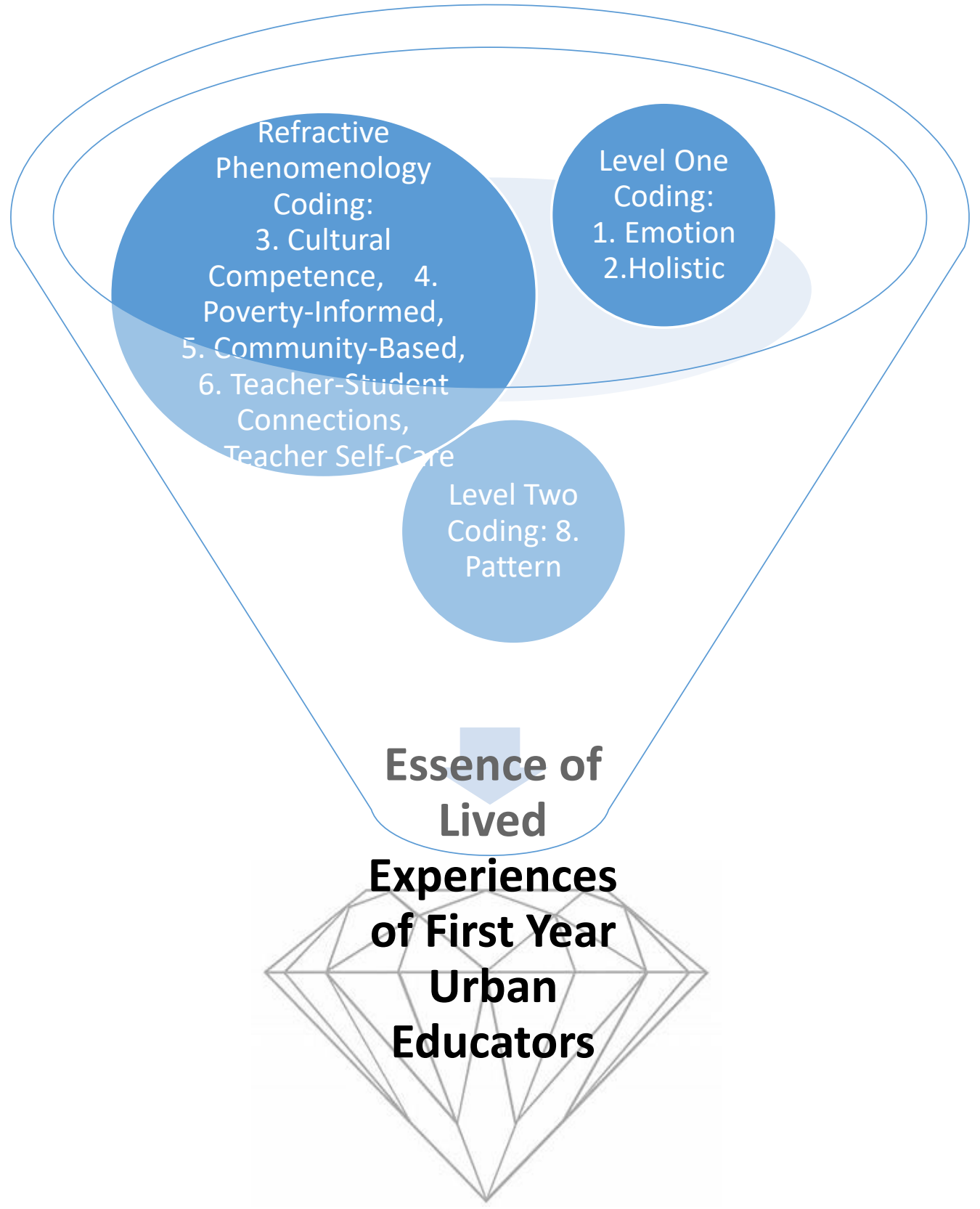


APPENDIX J

First Year Urban Teacher Induction Plan

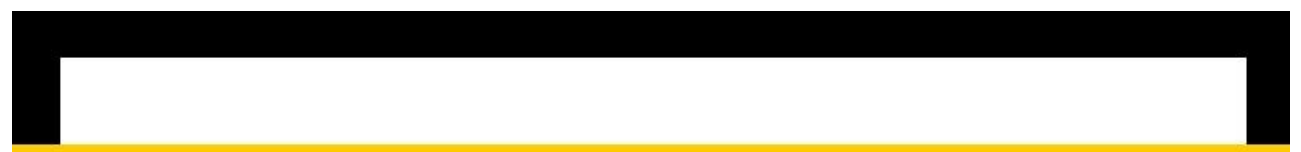

FIRST YEAR URBAN TEACHER INDUCTION PLAN

\section{PROJECT TITLE}

Urban-Ready, Willing, and Able: First Year Urban Teacher Induction Plan

\section{DESCRIPTION}

This yearlong program is designed to support urban teachers as they transition into classrooms for their first year of teaching in urban schools. The program provides these teachers with access to the expertise of multiple stakeholders who are skilled in urban education including, but not limited to, district and school administrators, experienced urban educators, representatives from local universities, and community partners. Participants will also benefit from the connections they form with fellow first year urban teachers.

\section{OBJECTIVE}

A multi-layered system of support for first year urban teachers that increases their abilty to effectively apply sound pedagogical practices and nurture an urban competencies skillset as they gain traction in their first year. The goal of the program is to encourage early successes in the classroom that increase new teacher retention rates.

\section{PLAN STRUCTURE}

- Teacher participants agree to engage as a yearlong cohort of monthly professional learning experiences where they may earn professional development credit or stipend.

- Professional experiences focus on topics such as cultural competence, poverty-informed practices, teacher self-care, and trauma-informed practice.

- Participants engage in their own action research project-based learning study that culminates in a presentation as part of the New Teacher Induction Showcase of Urban Teaching and Learning, a community event.

- Early hires are also invited to co-teach alongside experienced urban educators during existing summer programs.

- Sessions planned and facilitated by the Director of New Teacher Induction, Development, and Retention.

- The Director of New Teacher Induction, Development, and Retention will also collaborate with the Research Department to collect data on the economic impact of the program and to make revisions to ensure viability. 


\title{
CURRICULUM VITAE
}

Sandra L. Hogue

\section{Education}

Doctoral Candidate, Ed. Leadership Evaluation \& Organizational Development, University of Louisville

2008 Rank I in Literacy University of Louisville

2000 Master's of Education, University of Louisville

1995 Bachelor's of Science, University of Louisville

\section{Professional Experience}

August 2018 to Present, DISTRICT K-12 ELA INSTRUCTIONAL COACH Jefferson County Public Schools

Provide professional support for ELA instruction for teachers and schools across the district through facilitation of PLCs, development and delivery of need-specific professional development, and coach teachers, schools, and colleagues to improve ELA practices with a specialty in writing.

\author{
January 2007 to August 2018, GOAL CLARITY COACH \\ Jefferson County Public Schools \\ Collaborate with district and school level personnel to support best instructional \\ practices; develop writing and reading curriculum for elementary schools across the \\ district for the Common Core; conceptualize, develop and deliver professional \\ development for teachers and administrators
}

\section{4 to Present and 2006-2010, CO-DIRECTOR and TEACHER CONSULTANT of THE LOUISVILLE WRITING PROJECT (LWP) \\ University of Louisville \\ Co-develop and direct the Summer Institute experience; submit professional development sessions for approval in JCPS; write and develop professional development for schools, districts, and national audiences; staff developer in the National Writing Project for SEED II Grant to provide intensive professional development for underperforming urban}


schools; co-leader of JCPS collaboration with LWP to develop advanced skillset of Goal Clarity Coaches

\section{5-2018, SUMMER LITERACY BOOST TEACHER and SITE}

\section{INSTRUCTIONAL LEADER}

Jefferson County Public Schools

Served as team teacher - 2015; Served as site instructional leader - 2016-2018

monitoring instructional practices, coaching and training teachers, coaching practices,

and monitoring students

\section{Spring 2008, Fall 2008, Spring 2009, ADJUNCT INSTRUCTOR}

University of Louisville

Teaching Writing P-12 graduate level course; instructed pre-service and active teachers in pedagogy, best practice instruction, analysis of student work, etc.

\section{Summer 2008 to Summer 2009, ACES PROGRAM LITERACY INSTRUCTOR} Jefferson County Public Schools

Prepared second-career professionals to become classroom teachers

\section{Fall 2001 to Spring 2004, ADJUNCT INSTRUCTOR}

University of Louisville

Language Arts Methods; instructed pre-service teachers in pedagogy and best practice instruction for teaching elementary readers and writers

\section{5 to 2006, ELEMENTARY SCHOOL TEACHER}

Stonestreet Elementary Schools, Jefferson County Public Schools

Orchestrated and facilitated learning for students in multi-age classes; SBDM member;

Literacy Leader; Writing Cluster Leader, ILT member

2006, AUTHOR: BREAKTHROUGH TO MEANING: HELPING YOUR KIDS BECOME BETTER READERS, WRITERS, AND THINKERS by Clyde, Barber, Hogue, and Wasz Heinemann Publishers

Teacher-researcher engaged in exploring strategies to support literacy learning; coauthored published professional text.

2006, AUTHOR: HELPING STRUGGLING READERS AT ANY AGE DO WHAT GOOD READERS DO

Article published in Kentucky English Bulletin

\section{Professional Activities}

2000 to Present, CONFERENCE PRESENTER/CO-PRESENTER

- Fall 2018, Children's Literature that Encourages Advocacy, LWP Fall Mini-

Conference 
- Spring 2017, Summer Field-Based Collaborative Urban University and Urban School District Teacher Induction Experience, Spring Research Conference, Cincinnati, OH

- January 2015 and 2016, Read It Again! The Best of Children's Literature, LWP Winter Mini-Conference

- October 2014, Strategies for Checking for Understanding, ASCD Conference for Educational Leadership, Orlando, FL

- September 2014, Because My Momma Said So! Using Subtext and Other Strategies to Support Students in Developing Informed Opinions, Kentucky Writing Project Fall Conference

- June 2014, Protocols for Powerful Collaborative Planning, ASCD Conference on Teaching Excellence, Grapevine, TX

- January 2014, On-Demand Writing as Formative Assessment, LWP Winter MiniConference

- June 2010, Building the Writing Community with Close-to-Home Texts: Filling the Classroom with Texts that Anchor the Learning Community and Foster Resiliency, National Writing Project Urban Sites Conference, Louisville, KY

- September 2008, Teaching Persuasive Writing in the Writing Workshop, Kentucky Writing Project Fall Conference

- January 2007, Teaching Transactive Writing in an Open Genre Writing Workshop, LWP Winter Mini-Conference

- June 2006, Explicit Reading Strategy Instruction for Struggling Readers, Kentucky Writing Project Achievement Gap Institute. Jenny Wiley State Park

- August 2006, Explicit Reading Strategy Instruction for Struggling Readers, Dennis Wooton Elementary School

- June 2006, Using Graphic Organizers to Help Kids Before and During High-Stakes Testing, Kentucky Writing Project Achievement Gap Institute. Jenny Wiley State Park

- 2006, Kentucky Writing Advisory Committee, Kentucky Department of Education.

- December 2005, Explicit Reading Strategy Instruction for Struggling Readers, Pike County Title One Conference

- November 2001, 2002, 2003, 2004, 2005, 2006 Using Subtext and Visual Arts to Enhance Student Reading and Writing, National Council for Teachers of English (NCTE) Conferences

- January 2003, Using Subtext and Visual Arts to Enhance Student Reading and Writing, Hawaii International Conference on Education, Oahu, HI

\section{Awards and Other Professional Experiences}

- December 2016 Teach to Lead U.S. Department of Education Teacher Leadership Initiative, Chicago, IL

- Fall 2007 - Present, KTIP Mentor Teacher (12 teachers)

- 2013, Writing Task Force, Jefferson County Public Schools

- Summer 2015, Spoke on behalf of the National Writing Project on Capitol Hill in Washington, D.C. to lobby for i3 SEED funding 